\title{
CIPS Validation Data Plan
}

Nam Dinh

March 2012
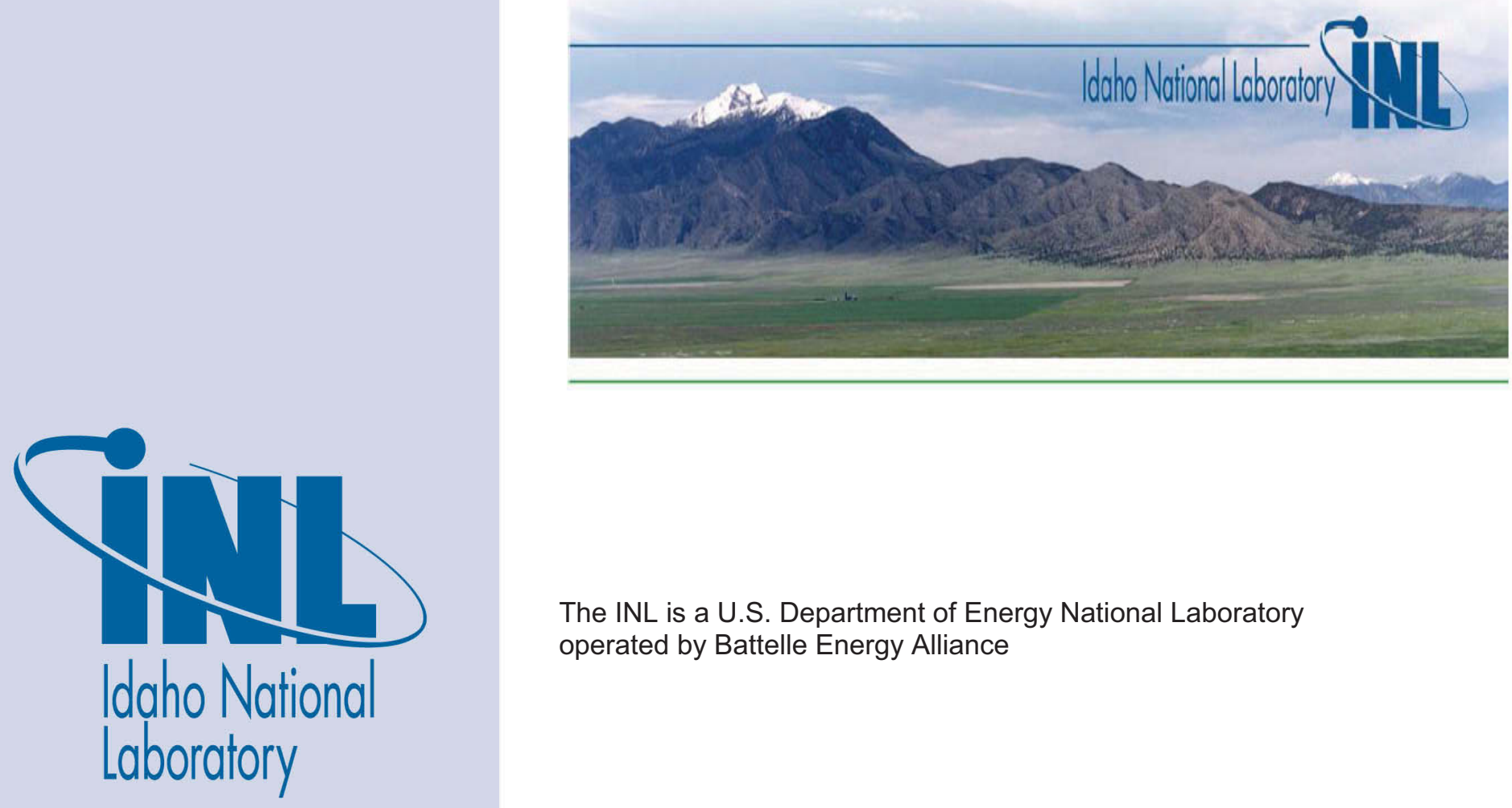

The INL is a U.S. Department of Energy National Laboratory operated by Battelle Energy Alliance 
INL/EXT-12-25347

\title{
CIPS Validation Data Plan
}

\author{
Nam Dinh
}

March 2012

\section{Idaho National Laboratory Nuclear Science \& Engineering Division Idaho Falls, Idaho 83415}

http://www.inl.gov

Prepared for the U.S. Department of Energy Office of Nuclear Energy Under DOE Idaho Operations Office Contract DE-AC07-05ID14517 


\section{NOTICE}

This information was prepared as an account of work sponsored by an agency of the U.S.

Government. Neither the U.S. Government nor any agency thereof, nor any of their employees, makes any warranty, expressed or implied, or assumes any legal liability or responsibility for the accuracy, completeness, or usefulness, of any information, apparatus, product, or process disclosed, or represents that its use would not infringe privately owned rights. References herein to any specific commercial product, process, or service by trade name, trade mark, manufacturer, or otherwise, does not necessarily constitute or imply its endorsement, recommendation, or favoring by the U.S. Government or any agency thereof. The views and opinions of authors expressed herein do not necessarily state or reflect those of the U.S. Government or any agency thereof. 
CIPS Validation Data Plan 


\section{ABSTRACT}

The Consortium for Advanced Simulation of Light Water Reactors (CASL) Program is a multi-institutional effort to develop and apply advance modeling and simulation (AMS) capabilities to the engineering analysis, design, and licensing of nuclear energy systems. One of CASL's tasks is to create AMS tools to accurately predict the extent and effect of crud deposits on nuclear power plants (i.e., crud-induced power shift). The current capabilities are derived from a series of codes, some mature industry products and some recently initiated by CASL. While there are many seemingly relevant experiments and measurement data, a close examination reveals that very few experiments and data are directly relevant to high-fidelity mechanistic models and the data are not compatible with modern verification, validation, and uncertainty quantification (VUQ) methods. In addition, because of long lead times and a lack of resources, only limited new data are expected over the next 3 to 5 years. This report documents the analysis, findings, and recommendations for developing a validation data plan to effectively deal with the severe lack and low quality of validation data. The study suggests a pragmatic approach whose near-term focus is support for the development of advanced capability in Virtual Environment for Reactor Applications. An application-oriented VUQ-guided data assimilation framework is envisioned as a means to integrate heterogeneous data to achieve uncertainty reduction. The study also identifies technical areas where further development in VUQ methods and tools are needed.

This report is prepared for the Department of Energy's Consortium for Advanced Simulation of LWRs (CASL) program's VUQ Focus Area under activity VUQ.VVDA.P4.02 entitled "Validation Data Planning for CIPS". 


\section{SUMMARY}

The Consortium for Advanced Simulation of Light Water Reactors (CASL) Program is a leading multi-institutional effort supported by the U.S. Department of Energy to develop and apply advanced modeling and simulation capability to create a virtual environment for predictive simulation of light water reactors (LWRs). The virtual reactor simulation is built on so-called Virtual Environment for Reactor Applications (VERA). The baseline capability (VERA-BL) comprises state-of-the-practice simulation codes made available to CASL by its industry partners. From this, the CASL researchers will use state-of-the-art methods and tools in nuclear fuel and material modeling, computational fluid dynamics and computational structural mechanics, radiation transport, computer and computational science, and verification, validation, and uncertainty quantification (VUQ) to develop advanced multi-physics, multi-scale, high-fidelity simulation capability or VERA advanced capability (VERA-AC). VERA-AC is envisioned to be based on more mechanistic coupled treatment of the physical processes at much higher resolutions than VERA-BL, and will subsequently be computationally intense and require solutions on modern supercomputers.

Programmatically, CASL focus is placed on a set of plant operational and safety "challenge problems." An improved understanding of these problems will benefit the safety case for reactor power uprate, increase fuel burnup, and contribute to plant life extension. One of the challenge problems identified is crud-induced power shift (CIPS). CIPS occurs in pressurized water reactors and is associated with the formation and buildup of a crud layer in fuel rods presumably caused by precipitation and deposition of boron and other chemicals and particulates (e.g., corrosion products). The formal CIPS challenge problem is

The CASL task is-by the year 2015-to develop and validate $V E R A$ methods that enable reducing axial offset anomaly ( $A O A)$ - the difference between predicted and measured core power's axial offsets (AO), from maximum $A O A 10 \%$ in the current practice, down to maximum $A O A$ 3\% over a fuel burnup cycle over (up to) $30 \mathrm{GWD} / \mathrm{MTU}$.

Central to the CIPS predictive capability is the mission to validate the models and methods developed for VERA-AC and ultimately, validation of the VERA integrated simulation capability. While there are many seemingly relevant experiments and plant measurement data, an initial review concluded that

- Very few experiments and plant data are directly relevant to and usable for validation of high-fidelity mechanistic models 
- Data that exist are not VUQ-grade (i.e. they are not compatible with modern VUQ methods)

- There is a severe lack of data to validate multi-physics, multi-scale capability

- Long lead times are required for the acquisition of any new applicable experimental data. Consequently, only a limited amount of VUQ-grade data may be expected from prospective experiments over the next $3-5$ years.

This "data realism" applies to all other CASL challenge problems, and the situation is germane to AMS in nuclear energy, including reactor engineering and plant safety. The common and pressing question is "how to effectively deal with the severe lack and low quality of validation data."

The goal of the present study is to develop a validation data plan (VDP) for VERA-AC applied to the CIPS challenge problem. The VDP is a dynamic planning instrument to guide and potentially optimize activities on data production and acquisition, data analysis and management, and data usage. At this time, VERA-AC requirements and the development plan are defined at a very high level, partly because of the technical challenges, uncertainty, and long lead time required for development and assessment of high-fidelity mechanistic simulation codes. Similar challenges exist in development of VUQ theory, methods, and tools, which is further complicated by the data deficit and resource constraints.

The following positions and principles will guide the development of the CIPS VDP:

- For CASL near- and medium-terms, the validation data task is directed to support pragmatic decision-making in developmental assessment of CASL products during the VERA-AC's formative phase (... as opposed to a "purist's" pursuit of validation of solidified products)

- "Data realism" (... as opposed to "data idealism") is central to the VDP. The provision is that by using advanced data strategies and VUQ tools, paramount information value can be extracted from community's knowledge base, including past experiments and legacy codes

- The VDP supports a structured, quantitative Bayesian framework for data assimilation strategy that exploits the "the whole is much more the sum of parts" principle to enable a multi-physics, multiscale validation pyramid

- Data support is an organic part of the VERA capability and most effective when VERA software seamlessly integrates validation databases 
- Validation data support is a community-scale effort across and beyond CASL and DOE-Nuclear Energy Programs. Future VERA users will greatly benefit from a systematic, collective, and continually growing knowledge base and databases.

Cutting through a large, multi-faceted, and evolving body of information about CASL/VERA/CIPS-related codes, models, and data, the present study's objective is to provide a systematic and, in some instances quantitative, delineation and ranking of gaps and issues anchored in validation-quality data or lack thereof. A VUQ-guided, application-oriented data assimilation framework is considered as an effective means to achieve uncertainty reduction through a Bayesian integration of heterogeneous information derived from simulation tools, a knowledge base, and available experimental and plant data. Industry/state-ofthe-practice simulation capability will be used to create a comprehensive knowledge/data base to aid calibration of models in the higher-resolution capability VERA-AC codes.

The study also suggests that CASL adopts a pragmatic (application-oriented) and holistic (communication) approach to validation and validation data. This means bringing to bear evidences and data of all types and all origins, developing infrastructure to house the data, developing methods for qualification and consistent treatment of the heterogeneous data, and developing methods for extracting more value from available experiments and datasets. In the proposed VDP, VERA simulation capability, validation activity, data and data support activity are tightly coupled through a CASL Data Center. The plan also includes a VUQ-guided use of code-to-code cross fertilization and a probabilistic integration approach. Such an approach can enable CASL to take advantages of the available capability set, overcoming limitations associated with immaturity of more mechanistic methods for multi-scale and multi-physics integration.

The approach proposed in this study can be embodied in the following technical recommendations for the VDP:

Establish the uncertainty of CIPS prediction by VERA-BL capability.

Establish a practical and scrutable workflow for applying VERA codes for CIPS challenge problem solving.

Establish and prioritize CIPS-specific requirements for integrated capability and for each component to guide development and assessment of VERA components.

Establish a CIPS-specific integrated calibration and validation plan.

Establish a CASL-wide policy, process, and infrastructure on validation data management including inventory, warehousing, processing, and controlled secure access.

Develop a "gap-tooth" scheme for integration of VERA-BL (e.g., neutron 


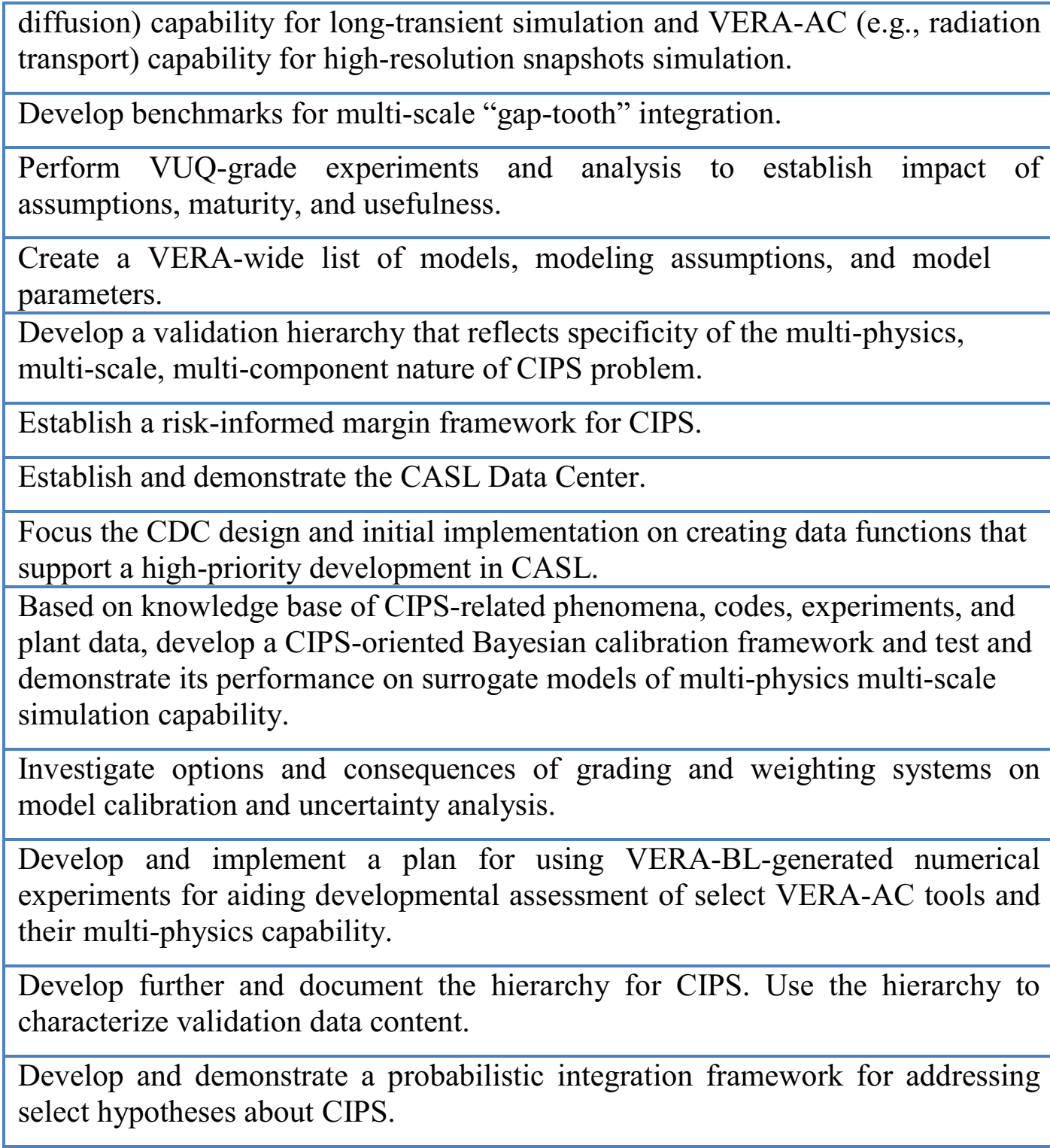




\section{ACKNOWLEDGMENTS}

This work has been authored by Battelle Energy Alliance, LLC under contract No. DE-AC07-05ID14517 with the U.S. Department of Energy. The U.S. Government retains a nonexclusive, paid-up, irrevocable, world-wide license to publish or reproduce the published form of this document, or allow others to do so, for U.S. Government purposes.

The work described in this report has benefitted from interactions with a number of collaborators in CASL, LWR-S and NEAMS programs. The author particularly appreciate the guidance, input and stimulating discussions on different aspects of the work detailed in this report that he has with the following individuals: Dr. Ralph Nelson (AMS), Dr. Robert Youngblood (INL), Dr. Robert Nourgaliev (INL), Professor Paul Turinsky (NCSU), Professor Richard Denning (OSU), Professor Qiao Wu (OrSU), Dr. Hyung Lee (Bettis), Dr. William Rider (SNL), Dr. Cetin Unal (LANL), Dr. Hongbin Zhang (INL), Dr. Anh Bui (INL), Dr. Binh Pham (INL), Dr. Brian Kendrick (LANL), Dr. Chris Stanek (LANL), and Dr. Robert Lowrie (LANL).

The author acknowledges Zeses Karoutas, Jefferey Secker and Yixing Sung, all of WEC, and Rosemary Montgomery (TVA) for sharing important insights and industry's perspectives on the CIPS challenge problem.

Dr. James Stewart (SNL), the CASL-VUQ Focus Area lead, has given this VDP task many constructive comments and periodic push.

Dr. Dana Kelly had made a critical influence on the author's interest and view on model calibration and code validation. 


\section{ACRONYMS}

\begin{tabular}{|l|l|}
\hline \multicolumn{1}{|l|}{} & \multicolumn{1}{|c|}{ Description } \\
\hline AC & Advanced Capability (in VERA-AC) \\
\hline AMA & Advanced Modeling Applications \\
\hline AMS & Advanced Modeling and Simulation \\
\hline ANC & Reactor Physics Code (Westinghouse) \\
\hline ANS & American Nuclear Society \\
\hline AO & Axial Offset \\
\hline AOA & Axial Offset Anomaly \\
\hline AP & Application Domain (CP-AP) \\
\hline BA & Boron and Additives \\
\hline BEPU & Best Estimate Plus Uncertainty \\
\hline BEPRU & Best Estimate Plus Reduced Uncertainty \\
\hline BISON & Nuclear Fuel Performance Code (INL) \\
\hline BL & Baseline (capability) \\
\hline BOA & Reactor Chemistry Code (boron analysis) (EPRI) \\
\hline BWR & Boiling Water Reactor \\
\hline C2C & Code-to Code (comparison) \\
\hline CASL & Consortium for Advanced Simulation of LWRs \\
\hline CC & Coolant chemistry and CRUD \\
\hline CDC & CASL Data Center \\
\hline CFD & Computational Fluid Dynamics \\
\hline CIPS & Crud Induced Power Shift \\
\hline CILC & Crud Induced Localized Corrosion \\
\hline CMFD & Computational Multiphase Fluid Dynamics \\
\hline COBRA & Subchannel Core TH Code \\
\hline CP & Challenge Problem \\
\hline CPTS & Challenge Problem Technical Specification \\
\hline CRUD & Chalk Rivers Unidentified Deposit \\
\hline CSAU & Code Scaling, Applicability, and Uncertainty \\
\hline DA & Data Assimilation \\
\hline DAKOTA & UQ and optimization software (SNL) \\
\hline DeCART & Radiation Transport Code \\
\hline DENOVO & Radiation Transport Code \\
\hline DNB & Departure from Nucleate Boiling \\
\hline DoE & Department of Energy \\
\hline DREKAR & CFD Code (SNL) \\
\hline EMDAP & Evaluation Model Development \& Assessment Process \\
\hline EMU & Experimental Measurement Uncertainty \\
\hline EPRI & Electric Power Research Institute \\
\hline & \\
\hline
\end{tabular}




\begin{tabular}{|l|l|}
\hline FA & Focus Area \\
\hline FC & Fuels and Claddings \\
\hline GTRF & Grid To Rod Fretting \\
\hline HRP & Halden Reactor Project \\
\hline HYDRA & CFD Code \\
\hline HYDRA-TH & HYDRA-based two-phase flow code (LANL) \\
\hline IET & Integral-Effect Test \\
\hline IFPE & International Fuel Performance Experiments \\
\hline INL & Idaho National Laboratory \\
\hline ITM & Interface Tracking Method \\
\hline LANL & Los Alamos National Laboratory \\
\hline LES & Large Eddy Simulation \\
\hline LIME & Lightweight Integrating Multiphysics Environment \\
\hline LWR & Light Water Reactor \\
\hline LWR-S & LWR Sustainability \\
\hline MAMBA & MPO Advanced Model for Boron Analysis (code) \\
\hline MC & Model Calibration \\
\hline M-C & Monte-Carlo \\
\hline MD & Molecular Dynamics \\
\hline MET & Multiple-Effect Tests \\
\hline MEV & Modeling, Experimentation, and Validation \\
\hline MIT & Massachusetts Institute of Technology \\
\hline MPO & Materials Performance and Optimization \\
\hline ND & Neutron Diffusion \\
\hline NE & Nuclear Energy \\
\hline NE-KAMS & NE Knowledge base for Advanced Modeling and Simula \\
\hline NEAMS & Nuclear Energy Advanced Modeling \& Simulation \\
\hline NPHASE & CMFD Code (RPI) \\
\hline NPP & Nuclear Power Plant \\
\hline NRC & Nuclear Regulatory Commission \\
\hline OECD & Organization for Economic Cooperation and Developme \\
\hline ONB & Onset Nucleate Boiling \\
\hline ORNL & Oak Ridge National Laboratory \\
\hline PCI & Pellet-Clad Interaction \\
\hline PCM & Predictive Capability Maturity \\
\hline PCMM & PCM Model \\
\hline PDF, pdf & Probability Distribution Function \\
\hline Peregrine & LWR Fuel Performance Code (INL) \\
\hline PIE & Post Irradiation Examination \\
\hline PIRT & Phenomena Identification and Ranking Table \\
\hline PIV & Particle Image Velocimetry \\
\hline PMO & Plant Measurements and Observations \\
\hline & \\
\hline
\end{tabular}




\begin{tabular}{|l|l|}
\hline POR & Plan of Record \\
\hline PWR & Pressurized Water Reactor \\
\hline QA & Quality Assurance \\
\hline QMU & Quantification of Margin and Uncertainty \\
\hline QOI & Quantity of Interest \\
\hline Q-PIRT & Quantitative (quantified) PIRT \\
\hline RBHT & Rod Bundle Heat Transfer \\
\hline RELAP & Reactor System T-H Code \\
\hline RISMC & Risk-Informed Safety Margin Characterization \\
\hline ROAAM & Risk-Oriented Accident Analysis Methodology \\
\hline RPV & Reactor Pressure Vessel \\
\hline RSU & Relevance, Scaling, and Uncertainty \\
\hline RT, RTM & Radiation Transport / RT Method \\
\hline SA & Sensitivity Analysis \\
\hline SC & State-of-the-practice codes (industry or commercial) \\
\hline SET & Separate-Effect Test \\
\hline SFB & Subcooled Flow Boiling \\
\hline SGS & Sub-Grid Scale (model) \\
\hline SNB & Subcooled Nucleate Boiling \\
\hline SNL & Sandia National Laboratory \\
\hline SQA & Software Quality Assurance \\
\hline SUA & Sensitivity and Uncertainty Analysis \\
\hline STH & System Thermal Hydraulics (code) \\
\hline TAMU & Texas A\&M University \\
\hline TCP & Technology Control Plan \\
\hline TH, THM & Thermal-Hydraulics / TH Method \\
\hline UQ & Uncertainty Quantification \\
\hline V\&V & Verification and Validation \\
\hline VD & Validation Domain (CP-VD) \\
\hline VDC & Validation Data Committee (working group) \\
\hline VDP & Validation Data Plan \\
\hline VDPI & Validation Data Product Integration \\
\hline VDMS & Validation Data Management System \\
\hline VERA & Virtual Environment for Reactor Applications \\
\hline VIPRE & Subchannel Core TH Code \\
\hline VOCC & Virtual Office, Computing, and Communication \\
\hline VRD & VERA Requirements Document \\
\hline VRI & Virtual Reactor Integration \\
\hline VVUQ, VUQ & V\&V and UQ \\
\hline V\&V & Verification and Validation \\
\hline WALT & Westinghouse Advanced Loop Testing \\
\hline & \\
\hline
\end{tabular}




\section{CONTENTS}

ABSTRACT $\quad 5$

SUMMARY 6

$\begin{array}{ll}\text { ACKNOWLEDGEMENTS } & 10\end{array}$

ACRONYMS 11

CONTENTS 14

1. INTRODUCTION 16

1.1. Trend and Challenges in Validation Data Support 16

1.2. CIPS - a CASL Challenge Problem 17

1.3. Validation and Data Activity in CASL 18

1.4. Validation Data Plan 20

1.5. Goal, Objectives and Approach of This Study 21

2. CIPS VDP TECHNICAL COMPONENTS EVALUATION 24

2.1. Challenge Problem Specification 24

2.2. Problem Solution Framework 26

2.3. Simulation Capability 29

2.4. VUQ Processes $\quad 32$

2.5. Validation Data an Database Support 38

2.5.1. Overview $\quad 38$

2.5.2. Radiation Transport and Reactor Physics $\quad 40$

2.5.3. Thermal-hydraulics 43

2.5.4. Coolant Chemistry and CRUD 47

2.5.5. Nuclear Fuels and Claddings 49

2.5.6. Multi-physics Simulation 51

2.5.7. Cross-cutting Issues 54

2.6. Programmatic Targets and Constraints 56

$\begin{array}{ll}\text { 2.7. Decision Model } & 57\end{array}$

3. CASL VALIDATION DATA STRATEGY 60

3.1. Validation in Nuclear Engineering Context 60

3.1.1. Validation from the Communication Standpoint 60

3.1.2. Validation from the Application Standpoint 61

3.2. Pragmatic Validation Data Strategy 64

3.2.1. CASL Data Center 65

3.2.2. Application-oriented Total Data Assimilation 68 
3.2.3. Qualification and Integration of Heterogeneous Data

3.2.4. Code-to-code Cross Fertilization

4. CIPS VALIDATION DATA PLAN: UPDATE AND IMPLEMENTATION

4.1. CIPS Phenomenological Hierarchy and Decomposition $\quad 79$

4.1.1. Multi-physics Multi-scale Hierarchy 79

4.1.2. Thermal-hydraulics (subcooled flow boiling) 80

4.1.3. Coolant Chemistry and CRUD 85

4.1.4. Thermohydraulics-CRUD coupling 86

4.1.5. Other Technical Areas and Couplings 88

4.2. CIPS VDP: update $\quad 89$

4.2.1. CIPS Predictability 89

4.2.2. Probabilistic Integration Framework 95

4.2.3. CIPS VDP Implementation: Case Study 97

5. CONCLUSIONS AND RECOMMENDATIONS 99

$\begin{array}{ll}\text { BIBLIOGRAPHY } & 103\end{array}$

$\begin{array}{ll}\text { APPENDIX } & 113\end{array}$

Remarks on Sensitivity and Uncertainty Analysis for CIPS 113 


\section{INTRODUCTION}

\subsection{Trend and Challenges in Validation Data Support}

Over the past two decades, Advanced Modeling and Simulation (AMS) have permeated virtually all corners of the field of engineering. Along with increasingly powerful computer simulation codes and a vested interest in using simulation results to support engineering decision-making, increasingly stringent requirements are being applied to solution and code verification, validation, and uncertainty quantification (V\&V-UQ). Nuclear energy, and nuclear reactor engineering specifically, are no exception. While the framework for code assessment provided by methods such as CSAU (NRC, 1988) and EMDAP (NRC, 2005) remains a valid high-level, largely qualitative guide, it has become clear that a more quantitative assessment of the numerical simulations is required. Over the past several years, a significant attention and encouraging progress were made in developing and applying modern V\&V-UQ methods and tools to nuclear energy applications, notably under the Nuclear Energy Advanced Modeling and Simulation (NEAMS) and Consortium for Advanced Simulation of LWRs (CASL) programs; see e.g. Nelson et al. (2010), Unal et al. (2011). In a previous work for CASL-VUQ (Dinh, 2011), specificity of nuclear reactor engineering simulation, experimentation, and validation was delineated. In particular, these recent studies point to critical gaps, both in quantity and quality, of experimental database for validation and uncertainty quantification (UQ) of advanced simulation codes. The trend toward a UQ goal imposes substantial requirements on experimentation, including assessment of measurement uncertainty and errors associated with experimental scaling, design, operation, data acquisition and processing.

In addition to the UQ trend, other facets of the AMS trend are summarized below along with ramifications for experimental validation support.

- Trend: Higher fidelity modeling (physics detail). This also substantially increases the number of models and model parameters.

Requires advanced experimentation (e.g., isolating individual mechanisms that govern CRUD formation) under conditions of relevance. 
- Trend: Higher resolution (temporal and spatial detail).

Requires advanced high-resolution diagnostics (typically, generating huge amount of data).

- Trend: Tight multi-physics coupling.

Requires multi-physics system testing (often in-pile / research reactor testing, or actual plant's events).

- Trend: Coupling of models of different fidelity (in system simulation). Requires integral experiments to test the coupling.

- Trend: Multi-scale integration, e.g. "gap-tooth" scheme, domain decomposition.

Requires experiments with "multi-scale" diagnostics to obtain data for testing "conditioning" (from coarse-to-fine-scale) and "homogenization" (using fine-scale simulation to inform coarse-grain model parameters).

\subsection{CIPS - a CASL Challenge Problem}

The Consortium for Advanced Simulation of LWRs (CASL) program is a leading multi-institutional effort supported by the U.S. Department of Energy to develop and apply advanced modeling and simulation (AMS) capability in a fashion that catalyzes qualitative changes in the practice of engineering analysis, design, and licensing of nuclear energy systems. Built on so-called Virtual Environment for Reactor Applications baseline capability or VERABL - comprised of state-of-the-practice simulation codes made available to the CASL by its industry partners, the CASL researchers bring to bear stateof-the-art methods and tools in nuclear fuel and material modeling, computational fluid dynamics and computational structural mechanics, radiation transport, computer and computational science, verification, validation and uncertainty quantification (VUQ) to develop advanced multiphysics, multi-scale, high-fidelity simulation capability, VERA-AC. The later is envisioned to base on more mechanistic coupled treatment of the physical processes, at much higher resolution than VERA-BL, computationally intense and hence requiring solutions on high-performance supercomputers.

Programmatically, CASL focus is placed on a set of plant operational and safety "challenge problems". An improved understanding of these problems can benefit the safety case for reactor power uprate, higher fuel burnup and plant life extension. Crud-Induced Power Shift (CIPS) was identified as a substantial challenge to be tackled by CASL during Phase I. CIPS occurrs in Pressurized Water Reactors (PWRs). It is associated with formation and buildup of a crud layer in fuel rods presumably caused by precipitation and 
deposition of boron and other chemicals and particulates (e.g., corrosion products) present in the primary coolant on the cladding surface in sub-cooled flow boiling region. The AMS prediction challenge lies with complex processes that govern CIPS, including multiple "classical" physics (core physics, fuel and cladding behavior, thermal-hydraulics, coolant chemistry), their delicate coupling (e.g., chemistry/ material/ thermal / fluid interactions upon crud formation), and span over many decades of length scale (nanometers to tens of meters) and time scale (microseconds to years). Given the engineering nature of the CIPS challenge problem, advanced simulation capability developed by CASL for CIPS necessarily involves modeling across contributing physics and different scales.

Central to the CIPS predictive capability is the mission to validate the models and methods developed for VERA-AC, and ultimately, validation of the VERA integrated simulation capability. While there are many seemingly relevant experiments and plant measurement data, their initial review as documented in (Dinh, 2011) concluded that

- Very few experiments and plant data are directly relevant to and usable for validation of high-fidelity mechanistic models

- Data that exist are not VUQ-grade (i.e. they are not compatible with modern VUQ methods)

- There is a severe lack of data to validate multi-physics, multi-scale capability, and

- Long lead time are required for the acquisition of any new applicable experimental data. Consequently, only a limited amount of VUQ-grade data may be expected from prospective experiments over the next 3-5 years.

This situation is not acceptable, given the CASL plan to develop and qualify coupled multi-physics tools for CIPS application by 2015 (the end of CASL Phase I).

\subsection{Validation and Data Activity in CASL}

The VERA-AC comprises a panoply of physics codes. Each code by itself is a sophisticated software, embodying a hierarchical system of models of physical processes at different scales. Assessment of these models requires having data from experiments such as single-physics, multi-physics, separateeffect, and integral-effect tests, as well as plant measurements (Figure 1.1).

It is noted that a broad range of validation tasks and data-related activities have been performed and are planned in Materials Performance and 
Optimization (MPO), Thermal-Hydraulics Methods (THM), Radiation transport Methods (RTM) and Advanced Modeling Applications (AMA) Focus Areas. Focusing on evaluation of CASL-developed models and VERA codes, the validation activities are applications-oriented model-centric and code-specific. Even for a given code, a systematic approach to the validation activity has yet to emerge. Any plan that exists is ad hoc based on expert judgments. There is neither structure nor a validation metric that could help measure progress and assess adequacy and feasibility of the testing/benchmarking plan. In fact, during the next few years, much of the advanced capability in CASL would still be under development, so the activity would more be "model calibration" and "developmental assessment" than "validation". The main goal in this development stage is to ensure that the developers choose models that are applicable for the intended application, and that the models and resulting codes can be assessed with available and projected (expected) data.

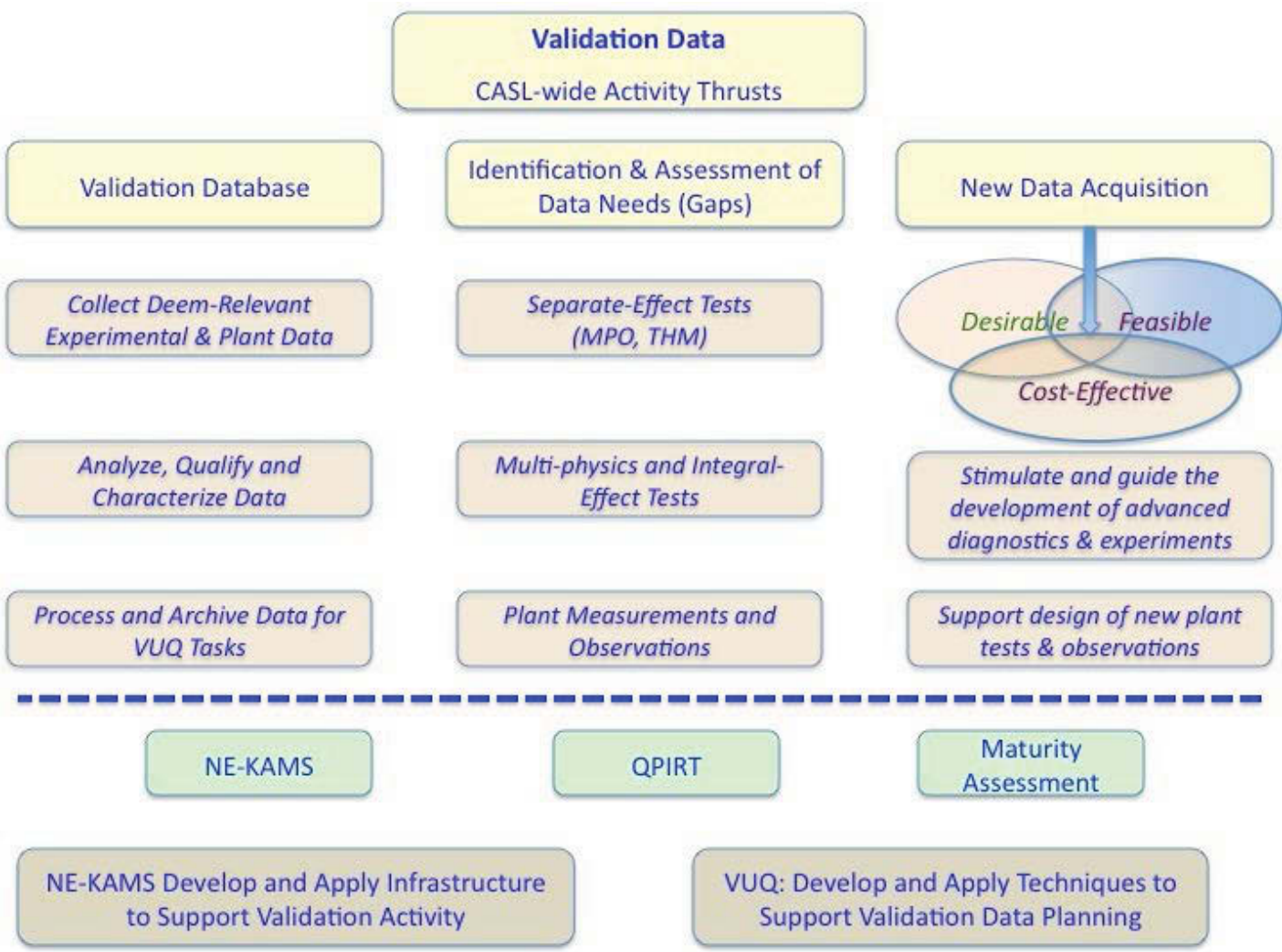

Figure 1.1. Overview of validation data activity in CASL (above dashed line). The validation data activity in CASL leverages on developments in NE-KAMS (Nuclear Energy - Knowledge base for Advanced Modeling and Simulation), QPIRT (Quantitative Phenomena Identification and Ranking Table) methods and tools, and PCMM (Predictive Capability Maturity Model). 
Within this "developmental assessment", benchmark tests, code-to-code comparisons, adjustments of parameters for better fits, etc. would all be necessary elements. However, experience has taught that for increasingly complex problems, the "common-sense" approach to tuning model parameters one-at-the-time would eventually go into such conflicts that even in the best scenario, the effort would be formidable. And even after a benchmark is completed, how this experience, success or failure, can be used in the task of specifying model-form and model parameter uncertainty? Such uncertainty values are needed when the AMS models are used in calculating a reactor case, where these uncertainties are propagated to determine the uncertainty of the integral quantity of interest.

While the common-sense approach to validation (including physics-byphysics, scale-by-scale efforts to benchmark models and codes against available experimental data, and to validation data, already practiced in CASL and outlined in Figure 1.1) must continue, it has become clear that by itself the approach would not be capable of bridging the gap between high-resolution models in advanced codes and deficient data support in a timely and effective manner.

\subsection{Validation Data Plan}

As first defined in (Dinh, 2011), Validation Data Plan (VDP) is a dynamic planning instrument to guide and, potentially, optimize activities on data production and acquisition (e.g., through new experiments or plant measurements), data analysis and management (e.g., qualification, classification/meta-data, archiving), and data usage so that they enable effective support for development, assessment and application of simulation tools intended for a challenge problem (see Figure 1.2).

Dinh (2011) outlines seven factors that affect the VDP formulation, namely:

(i) Challenge problem specification (mission and success criteria)

(ii) Problem solution framework and approach (which simulation codes, and how they, and their applicability)

(iii) Status of required capabilities in available and selected analysis tools

(iv) VUQ techniques and method for assessment of predictive capability

(v) Types, quality, availability, and accessibility of existing data 
(vi) Projected time and resources for generating new data

(vii) Decision model that integrates information from (i) through (vi) and prioritizes data activities, based on cost-benefit analysis of possible activities.

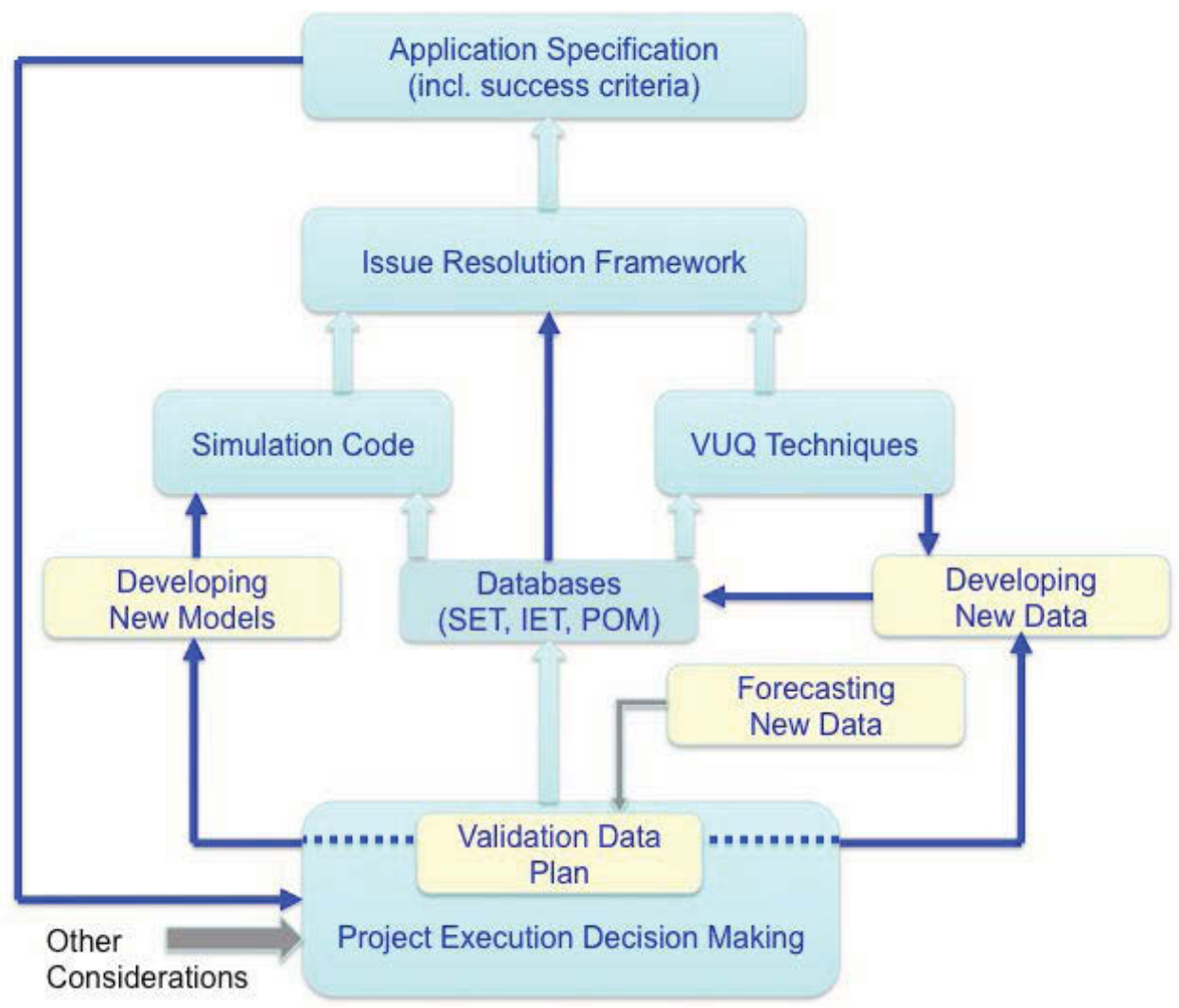

Figure 1.2. The project implementation chart for a specified application (challenge problem) involves an iterative cycle (in the figure, green arrows show a second round that my require development, validation, and implementation of new models, methods, and experimental data (Dinh, 2011). The validation data plan is part of the CASL project decisionmaking, which includes (and therefore must balance) several challenge problems concurrently pursued in CASL (Karoutas, 2011).

\subsection{Goal, Objectives and Approach of This Study}

Formulated as a task in VUQ, the present study is VUQ- driven datacentric, and to certain extent, also data-theoretic, as required to support formulation of the VDP for the VERA-AC (advanced capability) applied to 
the CIPS challenge problem. Through the CIPS case, the overarching goal of the present study is to

- Design a "practical" validation data support strategy that serves the CASL goal in applying modern VUQ methods for assessing VERA "predictive capability" for the challenge problems;

- Guide, facilitate and enable the implementation of this strategy.

In carrying out the work documented in this report, CASL personnel recognizes that VERA-AC requirements and the development plan are currently defined at this time only at very high level, partly because of formidable technical challenges, uncertainty and characteristically long lead times required for development and assessment of high-resolution mechanistic simulation codes. Similar challenges exist in development of VUQ theory, methods, and tools and are further complicated by data deficits and resource constraints. During CASL Phase I, model and code development activities in MPO, THM and RTM Focus Areas will continue to resort to legacy data, legacy codes, baseline capability, and other existing tools for guidance. This includes code-to-code comparison, which is considered necessary and useful among developers and practitioners, although the practice does not fit the "purist's" VUQ paradigm.

At this time, the motivation and technical objectives for this task in the VUQ FA is to bring together

(i) knowledge of the modern view and capability in VUQ

(ii) knowledge of physical processes that govern the CIPS

(iii) knowledge of codes, models, and data available, used, potentially

use

accessible, and/or being developed in CASL for CIPS prediction,

to devise a practical VDP that effectively supports the CASL's mission in CIPS applications.

This report is intended to be a summary of the VUQ study of validation data and a "living" document for the CIPS VDP. The document assumes that readers have a basic knowledge of nuclear reactor analysis, CIPS phenomena, practices of modeling and simulation in engineering, and are familiar with fundamental concepts in VUQ. The audience may include CASL investigators in any Focus Area as well as those in the community wishing to contribute to the CASL mission or follow up with CASL progress. Therefore, while addressing several technical capabilities and their underlying models in thermal-hydraulics, fuels, and crud chemistry, the discussion is kept at the conceptual level. This report does not include specific data sets or descriptions 
of modeling details, proprietary or otherwise. As a contribution to the CASL strategic plan discussion, the proposed approach to validation data can serve as framework for discussion between VUQ and other technical Focus Areas in CASL.

The study approach includes

a. A critical review of the status and developmental plan for all seven factors that affect formulation and implementation of a VDP

b. An integrated assessment of the VDP relative to the defined goal (of the driving application) to identify capability gaps in simulation, VUQ infrastructure and data support in case the gaps identified are not commensurate with resources programmatically available,

c. Revisiting the mission's strategy and plan (e.g., goals of the challenge problem and the development schedule of supporting methods/tools/data) particularly to identify approach to address any underlying inconsistency between modeling, experimentation, and validation (MEV)

d. A discussion of the update and implementation of the VDP.

Elaboration of (a-b) is documented in Chapter 2, elaboration of (c) and (d) is in Chapter 3 and 4, respectively. Information about the status of and development plan for the CIPS VDP's seven components reflects the author's best knowledge of work in CASL in February/March 2012. Chapter 5 provides a summary of findings and recommendations. 


\section{CIPS VDP TECHNICAL COMPONENTS EVALUATION}

In this Chapter, seven technical components of a VDP are examined with respect to the CIPS challenge problem.

\subsection{Challenge Problem Specification}

The CASL uses a set of challenge problems (CP) to focus and guide the development, assessment, demonstration, and application of CASL-selected and CASL-established capabilities and methodologies in advanced modeling and simulation. These challenge problems are tough nuclear reactor engineering and safety-related problems, for which VERA tools, baseline and advanced, are expected to generate results to improve understanding of operational and safety margins associated the challenge problems. This improved understanding is central to the risk assessment of potential for reactor power uprate and higher fuel burnup.

CIPS, the challenge problem in question, has been found to affect nuclear fuel management and impose operational constraints in a number of nuclear power plants in the U.S. CRUD has been observed to deposit on the surface of fuel pin cladding, most notably in areas of high coolant enthalpy. In the case of PWRs, this is the subcooled flow nucleate boiling region in the upper section of the core. The deposits have a complex structure and complex compositions, reflecting the complex chemistry of reactor coolant.

There is a lack of quantitative understanding of mechanisms that govern crud nucleation and growth. Of relevance to the reactor operation is boron uptake in crud deposits. A neutron absorber, this crud boron affects neutron flux in the core and hence reactor power distribution. When measurable amount of boron is accumulated in the upper span, it causes the neutron flux to shift axially downward (hence, Axial Oddset).

Inaccuracy in predicting the effect of boron-containing crud leads to Axial Offset Anomaly (AOA), i.e. a significant discrepancy between the predicted and measured $\mathrm{AO}$ in reactor core power shape. A detail description of the CIPS challenge problem, associated phenomena and state-of-the-practice in addressing this problem has been provided in CASL AMA reports, authored by industry's lead experts in this area, and hence not reproduced here (Karoutas, 2010). 
It is instructive to note that crud deposition and boron uptake are intrinsic phenomena in PWRs under high power density, prolonged fuel reload cycle, and use of soluble boron in reactor control. Therefore, the challenge problem is not about preventing crud deposition or boron uptake, but about predicting the extent and effect of these phenomena on reactor power to ensure reactor operation as designed. Viewed in this light, CASL's role is to create AMS tools that enable analysts/engineers to predict the crud effect with high accuracy (specific target below). This then allows the vendor/utility/plant engineers to optimize/control plant operation, which minimizes the impact of crud on safety margins.

The predictability of reactor power shift, or so-called Axial Offset (AO, in $\%$ ), compared to designed power profile, is the essence of the CIPS challenge problem.

The CASL task is - by the year 2015 - to develop and validate $V E R A$ methods that enable reducing the difference between predicted and measured core power's axial offsets (AO) from maximum $A O 7-12 \%$ in the current practice ${ }^{1}$, down to maximum AO 3\% over a fuel burnup cycle over (up to) 30000 $M W d / M t U$.

From the AMA standpoint and within the current industry's practice, the CIPS challenge problem mission and success criteria are clearly defined. From a VUQ standpoint, there are two questions related to specification:

(i) What are the uncertainties in values of axial offset that are measured and predicted in the current engineering practice?

(ii) What is the level of uncertainty required for the axial offset value predicted with the help of CASL-VERA capability?

\footnotetext{
${ }^{1}$ In example of AOA (included in CASL public materials; see e.g., Turner, 2011), AO predicted with state-of-the-practice tools has been shown to deviate from measured $\mathrm{AO}$ at about $5 \mathrm{GWD} / \mathrm{MTU}$ into the burnup cycle, with under-prediction reached $7 \%$ (i.e. AO measured at nearly $10 \%$ vs predicted $2.6 \%$ ) between $10-15$ GWD/MTU before returning to a sharp over-prediction at the cycle end. In another example provided by R. Montgomery (SAMSI www, September 2011), AO is measured to reach a plateau of $12 \%$ in middle of the cycle (burnup $\sim 10$ GWD/MTU), where AO was predicted to be less than $4 \%$. The prediction again fails to capture the return to over-prediction at the cycle end, now with difference in AO as much as $13 \%$. These examples suggest that boron deposition is under-predicted for these cores, resulting in pronounced CIPS. In an apparent response to this, chemistry/crud models were recently modified that reversed the trend, now predicting excessive crud deposition over a large surface area, not observed in operating cores.
} 
The first question reflects infancy of UQ practice in nuclear reactor engineering, and answering it would be an important first step on the path to "quantitative assessment of calculations". This in itself is a substantial work, requiring access to underlying models, and a wide range of experiments and proprietary data.

The answer to the second question will help determine the quality required of the CASL solution and will guide the development of AMS capability, VUQ methods and validation data.

Recommendation 2.1: Establish the uncertainty of CIPS prediction by VERA-BL capability. This baselining is critical for measuring progress in VERA-AC development, and for strategic planning including $V D P$.

\subsection{Problem Solution Framework}

CIPS prediction requires the capability to simulate year-long operational transients over the fuel burnup cycle, with accounting for crud removal during shutdown periods. The simulation capability includes core power (reactor physics), thermal-hydraulics, coolant chemistry (corrosion products generation and transport), crud deposition and growth, and behavior of nuclear fuel and cladding. In the current practice, the CIPS AOA is calculated using the industry-developed computer codes, ANC (core power), VIPRE (core thermal-hydraulics), and BOA (coolant chemistry and crud). The BOA simulation tracks the plant system over burnup cycles, whereas ANC and VIPRE calculations are snapshots, performed for select time moments under a quasi-steady state assumption, using input from BOA for distribution of crud thickness and composition over the core.

Relative to the CIPS challenge problem, as implemented through past activities, CASL's current strategy effectively calls for

a) bringing state-of-the-practice industry-developed baseline capabilities BL (ANC, VIPRE-W, BOA) into a coupled system VERA-BL; performing assessment;

b) bringing state-of-the-art available capabilities VERA-SC, including commercial and research codes (Star-CCM+, DeCART, COBRA, Drekar) into a couple system VERA-SC; performing assessment;

c) developing advanced capabilities AC (MAMBA, Peregrine, HydraTH, SCALE, Denovo); 
d) enabling steps (a-b-c) with LIME/Trilinos-DAKOTA;

e) developing super-high-resolution simulation capability (when mature, this capability is used to inform a small, deem-critical set of sub-gridscale models in VERA-AC; e.g. bubble dynamics in sub-cooled flow boiling).

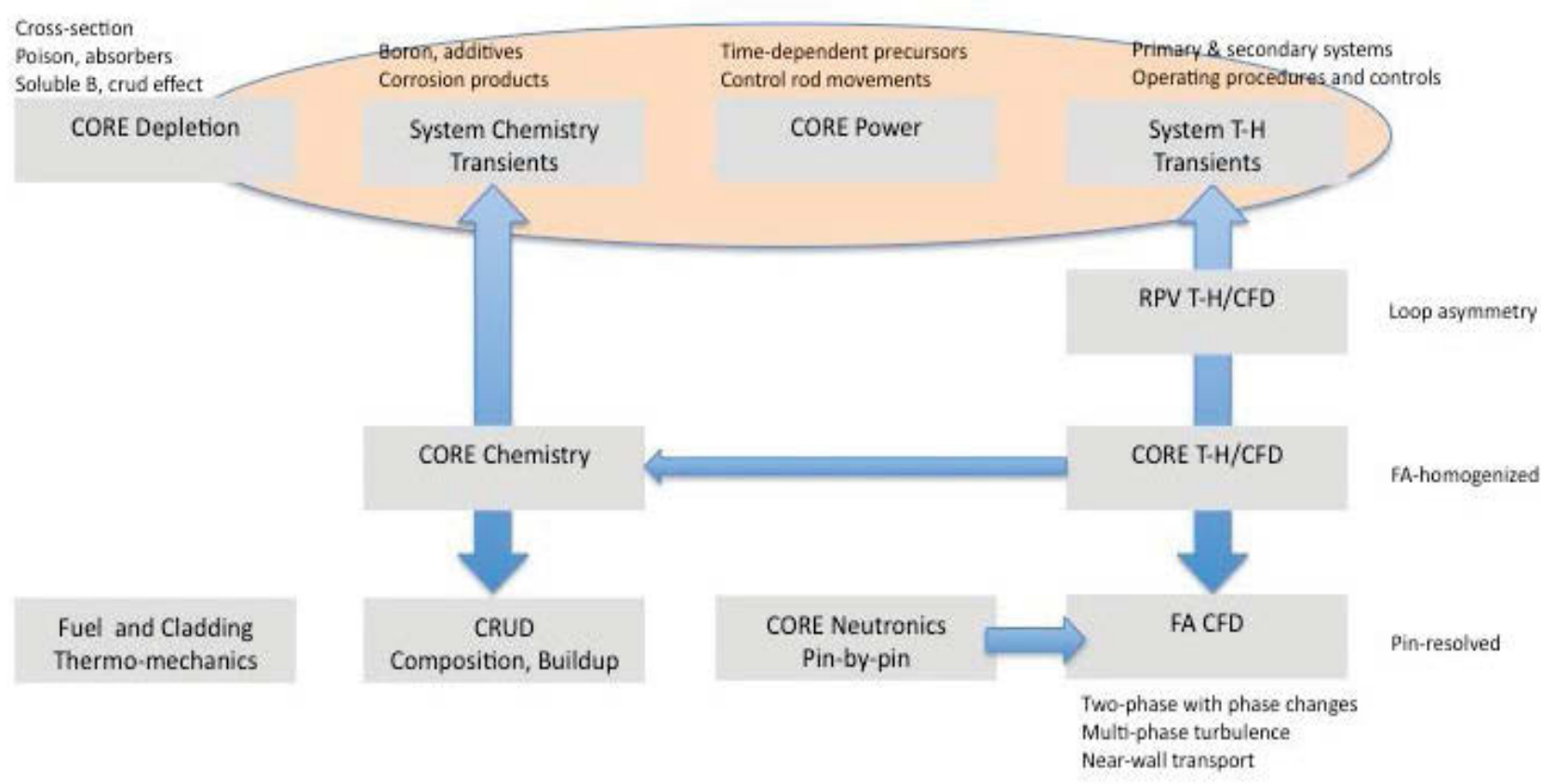

\begin{tabular}{|l|l|l|l|l|}
\hline & Description (Scale) & Physics (examples) & Length scale & Modeling \\
\hline A & Atomistic phenomena & Molecular interactions & $<\mu \mathrm{m}$ & Closure model to M \\
\hline M & Microphysical phenomena & Nucleation, wetting & $<\mathrm{mm}$ & Closure model to E \\
\hline E & Engineering/elementary unit & Control volume & $\mathrm{mm}$ to $10 \mathrm{~cm}$ & LES \\
\hline C & Component & Sub-channel, channel & $\mathrm{cm}$ to $\mathrm{m}$ & CMFD \\
\hline S & Sub-system & Fuel assembly, core, ANS/DeCART, VIPRE & $0.3 \mathrm{~m}$ to $10 \mathrm{~m}$ & Sub-system-level code \\
\hline F & Full system & Primary reactor system, plant, RETRAN/RELAP, BOA & $1 \mathrm{~m}$ to $100 \mathrm{~m}$ & System-level code \\
\hline
\end{tabular}

Figure 2.1. CIPS physical processes, components and scales involved.

Comment \#SF.1: Presently, the named capabilities are brought under a common LIME-based platform. However, no capability-integration framework has been articulated on how the VERA capabilities, once developed and assessed, are going to be used to achieve the challenge problem's goal, namely to reduce AOA to below 3\%. In other words, a VERA-AC workflow for the CIPS challenge problem is needed. 
Comment \#SF.2: Effectively, the activity plan supports a notion that improved predictability (e.g., of AOA in CIPS) is to be achieved by using simulation codes that are based on lower-time-scale and lower-length-scale models. However, this presents a formidable challenge for the CIPS challenge problem timeframe (2015), because it necessarily takes a long time and substantial resources to calibrate lower-length-scale models/codes (like MAMBA, Peregrine, Denovo, and Hydra-TH). Further, the VERA-AC codes, particularly Hydra-TH (multiphase CFD), Denovo (pin-resolved neutron transport) and Peregrine (fuel thermo-mechanics) can only at best run (simulation) for short time intervals ${ }^{2}$, not for simulation run over the burnup cycle as needed for CIPS AO prediction. Thus there is a wide gap between the time scales that are affordably covered by a prospective VERA-AC capability and the time scale of physical processes whose simulation is required for CIPS prediction.

Comment \#SF.3: The current selection of VERA-SC and VERA-AC codes reflects a basic understanding of CIPS multi-physics. However, the selection is based on availability of tools and expertise, but not driven by uncertainty ranking. There are modeling assumptions (e.g., interface between MAMBA and Hydra-TH; interface between Hydra-TH to system $\mathrm{T}-\mathrm{H}$ ) that have very limited to no support data thus introducing significant uncertainties, potentially more than the amount of uncertainty that could be reduced by using lower-length-scale models.

Comment \#SF.4: In the current arrangement (Figure 2.2), data and database (right column) are collected and used to benchmark and validate physics simulation codes. However, validation data and validation outcome (failure, or success) are not integrated in the CIPS problem solution.

\footnotetext{
${ }^{2}$ Also referred to in this report as "snapshot calculations".
} 


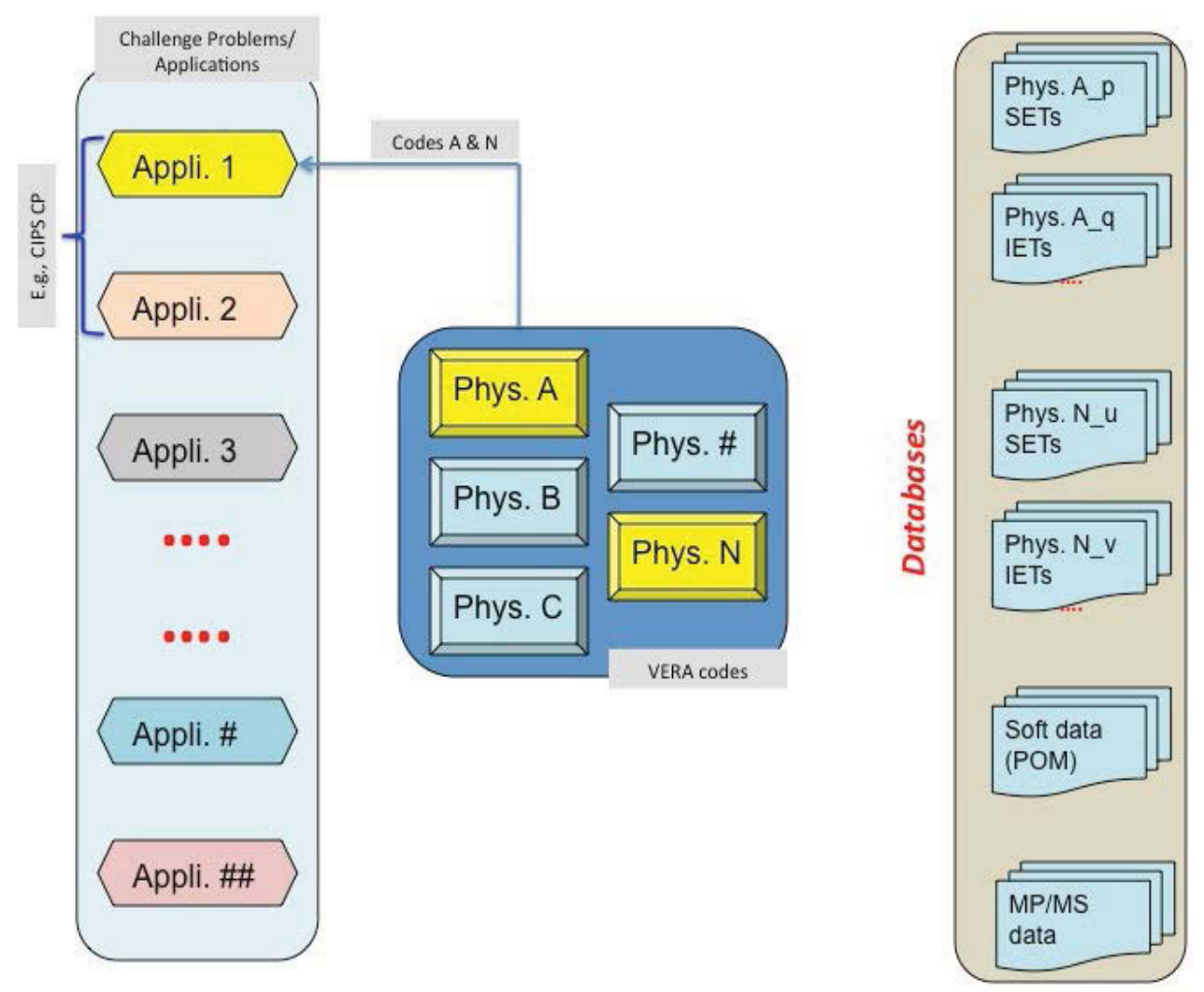

Figure 2.2. The current code application arrangement.

An application (CIPS simulation) requires the use of a subset of VERA codes, which (presumably) have been assessed, calibrated and validated, using experimental data. In the current arrangement, the validation activity (including data assimilation) is performed on individual test-bytest basis, and separated from the application, making it difficult to appropriate the significance of particular datasets and results of benchmark for the application.

Recommendation 2.2: Establish a practical, and scrutable workflow for applying VERA codes for CIPS challenge problem solving.

\subsection{Simulation Capability}

Table 2.1 (left column) depicts an early set of requirements (R.1...R.9) for CIPS challenge problems, as specified by CASL-AMA (Karoutas, 2010). The set's sufficiency and consistency was not scrutinized. The middle and right columns list capability/codes related to listed requirements (with comments on issues in the code's development and assessment), and uncertainty sources. 
Presently, CASL simulation capability is comprised of a panoply of codes. Some are mature industry products (ANC, VIPRE, BOA), others are research tools developed for other (not CIPS) applications or whose development (e.g., MAMBA, Hydra-TH) was recently initiated by CASL (see Table 2.2 below, also for highlight of their limitations and advantages). The above codes, including the newly formulated ones, are (were) developed as single-physics (e,g., neutronics, T-H) to capture an application domain. At best, these codes were assessed as stand-alone simulation capability.

Among the codes shown in Table 2.2 is a VERA-AC group of advanced capability codes (Denovo, Hydra-TH, Peregrine, MAMBA). Denovo and Peregrine are in their early developmental assessment phase. MAMBA and Hydra-TH are in their developmental formulation. An assessment plan for these codes is also being developed. 
Table 2.1. CIPS challenge problem's technical specification (CASL-AMA, 2010).

\begin{tabular}{|c|c|c|}
\hline CIPS Requirements (Objectives) & Capability/Cod, Development and Assessment Issues & Uncertainty sources, modeling assumptions \\
\hline $\begin{array}{l}\text { (R.1) 3D pin power prediction with } \\
\text { TH and Chemistry (boron) feedback }\end{array}$ & $\begin{array}{l}\text { Denovo. Computationally demanding for full- or } \\
\text { quarter-core, pin-resolved simulation. }\end{array}$ & \\
\hline (R.2) Model at least $1 / 4$ core & Quasi-steady simulation. & $\begin{array}{l}\text { Asymmetric loop. No capture transient } \\
\text { scenarios (with flush of corrosion products) }\end{array}$ \\
\hline $\begin{array}{l}\text { (R.3) Need full depletion including } \\
\text { boron in the crud }\end{array}$ & & $\begin{array}{l}\text { Cross sections for CRUD (crud composition; } \\
\text { thickness, location) }\end{array}$ \\
\hline (R.4) Account for subcooled boiling & $\begin{array}{l}\text { Hydra-TH. } \\
\text { Connection to surface. }\end{array}$ & $\begin{array}{l}\text { Surface, coolant/material chemistry effect on } \\
\text { nucleation, wettability, meniscus dynamics. }\end{array}$ \\
\hline $\begin{array}{l}\text { (R.S) Model grid spacer effects on } \\
\text { crud deposition }\end{array}$ & $\begin{array}{l}\text { This may require capability in turbulent multiphase } \\
\text { flow CFD with detail modeling of near-wall transport }\end{array}$ & $\begin{array}{l}\text { Turbulence model in multiphase flow. } \\
\text { Mass transfer in near-wall, boiling regions }\end{array}$ \\
\hline (R.6) Predict 3D crud distribution & $\begin{array}{l}\text { MAMBA. } \\
\text { Connection between } \mathrm{BOA} \text { and Hydra-TH. }\end{array}$ & $\begin{array}{l}\text { Deposition, precipitation mechanisms. Flow } \\
\text { and temperature effect. }\end{array}$ \\
\hline $\begin{array}{l}\text { (R.7) Uncertainty on total AO } \\
\text { prediction is less than } 3 \%\end{array}$ & $\begin{array}{l}\text { This requires an integrated multi-physics simulation } \\
\text { capability, long transient, and/or analyze hypotheses }\end{array}$ & $\begin{array}{l}\text { Not-AMS-related sources of uncertainty, e.g. } \\
\text { scenario hypotheses, boundary conditions }\end{array}$ \\
\hline $\begin{array}{l}\text { (R.8) Minimize calibration for } \\
\text { individual plants }\end{array}$ & $\begin{array}{l}\text { Only when CIPS uncertainty is dominated by } \\
\text { previously unresolved generic phenomena (not when } \\
\text { CIPS unpredictability is controlled by other factors, } \\
\text { e.g. plant operation }\end{array}$ & $\begin{array}{l}\text { Plant-specific scenarios/conditions matter, } \\
\text { including operational transients, shutdown } \\
\text { operations (e.g., crud removal) }\end{array}$ \\
\hline $\begin{array}{l}\text { (R.9) Predict in-core instrument } \\
\text { response }\end{array}$ & & \\
\hline
\end{tabular}

The code development plan itself is also subject to uncertainty. In part, this situation stems from a lack of clarity in the definition of scope, functional and performance requirements, and testing plan for the codes. An apparent conflict exists between the intent to use a specific technical challenge (e.g., CIPS) to drive the capability development and the desire to have a more versatile software product that can be used in a broad class of applications ${ }^{3}$.

\footnotetext{
3 At the time of this writing, the "VERA Requirements Document" (VRD) - originally developed by the AMA team in FY2010, is being updated with contributions from other Focus Areas, taking into account the collective experience in VERA development (Hess et al., 2012). Keeping in mind both industry's needs, technical feasibility and programmatic constraints, the VRD revision focuses on developing a set of high-level "foundational requirements" for VERA-AC in respective areas of nuclear physics, thermal hydraulics, fuel rod thermo-mechanics, structural materials, and chemistry, and in multi-physics coupling. For CIPS, it is judged that dominant physics are coolant chemistry, crud characteristics, thermal-hydraulics, core neutronics, and fuel/cladding thermo-mechanics.
} 
Table 2.2. CASL VERA codes of potential relevance to CIPS prediction.

\begin{tabular}{|c|c|c|c|c|}
\hline Physics & Capability & Output coupled to & Main issues & Potential advantage \\
\hline $\begin{array}{l}\text { Core } \\
\text { neutronics }\end{array}$ & $\begin{array}{l}\text { ANC9 } \\
\text { DeCART } \\
\text { Denovo }\end{array}$ & $\begin{array}{l}\text { VIPRE, BOA } \\
\text { Star-CCM+ } \\
\text { Drekar, Hydra-TH }\end{array}$ & $\begin{array}{l}\text { Not pin-resolved } \\
\text { Snapshot calculations }\end{array}$ & Pin-level resolution \\
\hline System T-H & RELAP5 & $\mathrm{BOA}$ & & $\begin{array}{l}\text { Account for system transients; provide } \\
\text { improved plant system conditions to } \mathrm{BOA}\end{array}$ \\
\hline $\begin{array}{l}\text { Fuel- } \\
\text { assembly T-H }\end{array}$ & $\begin{array}{l}\text { VIPRE-W } \\
\text { COBRA }\end{array}$ & ANC & & Allowing full-core simulation \\
\hline CFD/CMFD & $\begin{array}{l}\text { Star-CCM+ } \\
\text { Hydra-TH }\end{array}$ & $\begin{array}{l}\text { DeCART } \\
\text { Denovo, Peregrine }\end{array}$ & Snapshot calculations & \\
\hline $\begin{array}{l}\text { Coolant } \\
\text { chemistry }\end{array}$ & $B O A$ & MAMBA & $\begin{array}{l}\text { Uncertainty in modeling of corrosion, } \\
\text { corrosion product transport, effect of } \\
\text { system transients, loop asymmetry }\end{array}$ & \\
\hline CRUD & $\begin{array}{l}\text { BOA } \\
\text { MAMBA }\end{array}$ & $\begin{array}{l}\text { ANC, Denovo } \\
\text { BOA, Denovo }\end{array}$ & $\begin{array}{l}\text { Modeling assumptions about the } \\
\text { effect of local T-H conditions }\end{array}$ & \\
\hline $\begin{array}{l}\text { Fuel thermo- } \\
\text { mechanics }\end{array}$ & Peregrine & MAMBA, Denovo & Snapshot calculations & \\
\hline $\begin{array}{l}\text { Multi-physics } \\
\text { coupling }\end{array}$ & LIME & & $\begin{array}{l}\text { Different time-stepping requirements } \\
\text { for different physics }\end{array}$ & $\begin{array}{l}\text { Non-linearly coupled T-H/CFD, neutronics, } \\
\text { and fuel thermo-mechanics }\end{array}$ \\
\hline
\end{tabular}

\section{Recommendation 2.3: Establish and prioritize CIPS-specific requirements for integrated capability and for each component, to guide development and assessment of VERA components.}

\subsection{VUQ Processes}

Figure 2.3 depicts two domains where VUQ methods and tools are applied to support the challenge problem solution. This domain separation emphasizes the specificity of nuclear reactor engineering simulation (as discussed in Dinh, 2011), distinguishing "experiments" from the application's "reality".

Application Domain. In the Application Domain, the VUQ tools (e.g. DAKOTA) are coupled with simulation capability to support sensitivity analysis and, ultimately, quantification of uncertainty in predicted system behaviors (QOIs). For the three major contributions to uncertainty (parameter, model-form, and numerical), the most advanced are the VUQ methods and tools for propagating input and model parameter uncertainties. Investigations and developments are undertaken in CASL VUQ and elsewhere to further improve these methods and tools, making them more computationally 
efficient, as well as extending the methods to multi-physics, multi-scale simulation problems. In any case, model parameter uncertainty must be evaluated in the Validation Domain and provided as input for the Application Domain's VUQ.

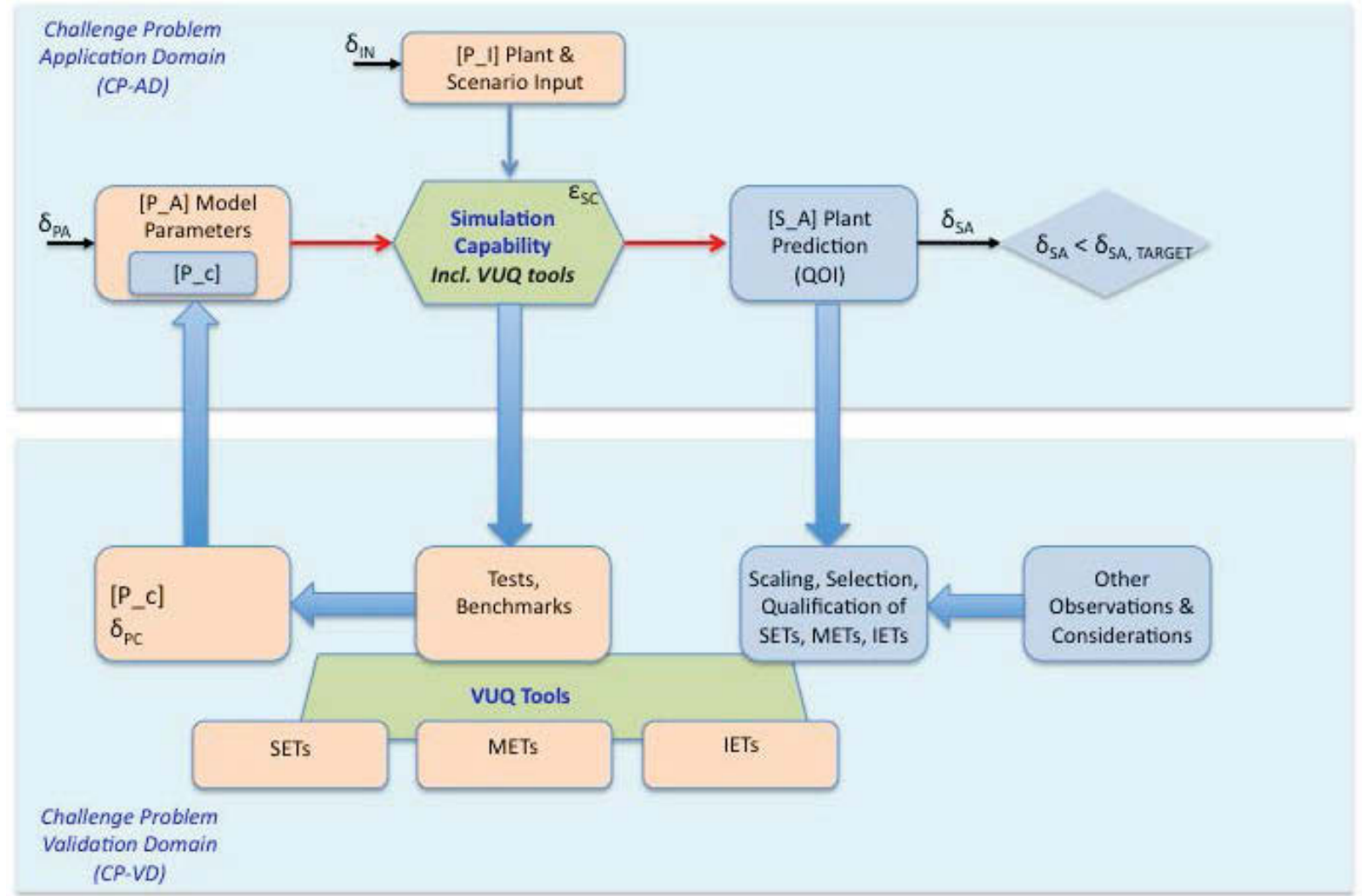

Figure 2.4. Challenge Problem's Application Domain (CP-AD) and Validation Domain (CP-VD). There are two separate instances (in AD and VD) where VUQ methods and tools are required ${ }^{4}$. $[P \ldots A]$ is the complete set of parameters needed for executing simulations in $A D .[P-c]$ is the specific set of parameters that are subject to calibration in $V D$.

4 Development of VUQ methods and tools focuses on forward problems, i.e., uncertainty propagation in CP-AD. It assumes a mature advanced simulation tool, e.g., VERA-AC and that model parameter uncertainties are readily established. Less attention and resources are given in to address inverse /optimization/ calibration problems in CP-VD. 
Currently, efforts are made in CASL to enable coupling of VERA baseline tools with DAKOTA, so the CIPS prediction can be made with UQ (modelparameter uncertainty) by the end of CASL Phase I (year 2015). Given a large number of models (and model parameters) involved in CIPS prediction, the main obstacle is to determine input (model-parameter) uncertainty needed for this UQ.

For quantification of model-form uncertainty, the methodology is less developed, particularly for complex multi-physics systems. The situation applies both to VUQ processes to be used in the Application Domain, and to what may be required from the Validation Domain.

Another challenge in this domain is quantification of the effect of numerical errors, especially for simulations that involve "legacy" codes and codes whose development and assessment do not adhere to modern standards in code and solution verification. Interactions of numerical errors with other forms of uncertainty can greatly complicate the UQ. There is a need to promote and institute verification culture in CASL.

Additional comments on requirements for sensitivity and uncertainty analysis methods and tools to support CIPS CP are provided in Appendix A.

Validation Domain. While a wide range of validation activities is currently undertaken and planned in the CASL Focus Areas, the Validation Domain work boils down to performing tests and benchmarks for VERA-BL and VERA-SC tools, both individually and in some cases as coupled tools. For VERA-AC tools, initial sets of tests and benchmarks are also defined. The setting in which this validation activity is performed is still evolving, but currently it is exercised in CASL in the frame of confidence building; [see "forward regime" in (a) below].

An instrumental component of the validation plan is to bring modern VUQ machinery to support this activity, making it more effective in meeting the CASL's objective in "qualitative assessment of calculations" (such as demonstrating the case that VERA tools enable achieving uncertainty in prediction of CIPS axial offset being less than 3\%). Thus, it is expected that the modern VUQ methods and tools, e.g., again, DAKOTA, are used in the Validation Domain, in two regimes.

(a) In a "confidence-building" forward regime, developed capability is benchmarked against experimental data and other observations. The test facility and embedded experimental processes are modeled, simulated, and analyzed, including sensitivity/uncertainty analysis.

(b) In an inverse regime, the VUQ tools are used in "optimization" mode to support best-estimating of model parameters and evaluation of model- 
parameter uncertainties, that enable the UQ-amenable use of the developed capability in the Application Domain.

Currently, VUQ-related efforts pursued in CASL (e.g., by VUQ, AMA, THM staff) are wide spread, mirroring the number of challenge problems investigated (CIPS, CILC, GTRF, PCI), the breadth of physics involved, the number of computer codes used, and the variety of VUQ needs and techniques.

The CASL VUQ-related efforts can be grouped into three categories:

(A) Application of state-of-the-art VUQ methods and tools to select computer codes (in RTM, THM/CFD), focusing on their basic functions (not necessarily specific to conditions of a challenge problem).

(B) Application of existing VUQ methods and tools to select computer codes to assess a code's capability with regard to its potential performance under conditions specific to treatment of a particular challenge problem.

(C) Adaptation and development of VUQ methods and tools to meet more advanced requirements for complex (e.g., multi-physics, multi-scale) problems characteristic of CASL challenge problems.

Of interest to this study (CIPS VDP) is a CIPS-related subset of Category (B) activity. This includes VUQ work on industry's codes (VIPRE, BOA, ANC), as well as considerations to formulate the VUQ plan for emerging MPO and THM developments (MAMBA and Hydra-TH codes). Of greater interest for the codes in their developmental phase are VUQ support for more effective model selection and model parameter calibration. The set of tests and benchmarks for CIPS-related physics codes is discussed in next Section 2.5. Notably, only a small subset of tests/benchmarks is directly relevant to CIPS conditions. Furthermore, validation/calibration exercises are selected on an ad hoc basis and carried out as an individual task. Figure 2.4 depicts a typical activity on code validation and parameters tuning practiced and associated issues (caption) that under-utilizes the information value of data generated in both code simulations and experiments. For CIPS, VUQ activity is not sufficiently coherent and comprehensive, to support the challenge problem's objective as stated in Section $2.1^{5}$.

Figure 2.5 shows a (desired) sequence of increasingly advanced and stringent treatments of CIPS calculated results, with implication on required VERA capability, VUQ processes, and supporting data. Situation (1) in the

5 Validation plan is under revision as a component of "CRUD Challenge Problem Product Integration" (J. Secker, Z. Karoutas, March 13, 2012). 
Figure represents the current state of practice in CIPS analysis that QOI prediction is provided without quantification of uncertainty. Situation (2) represents implementation of Recommendation 2.1 (about establishing the baseline's uncertainty). Situation (3) shows a larger spread of uncertainty expected for VERA-AC's early developmental phase. Situation (6) represents a full-scope UQ, resulting in probability density function (pdf) of QOI. Note that CIPS prediction requires a combined use of high-fidelity VERA-AC and lower-fidelity VERA-BL (or its enhanced version).

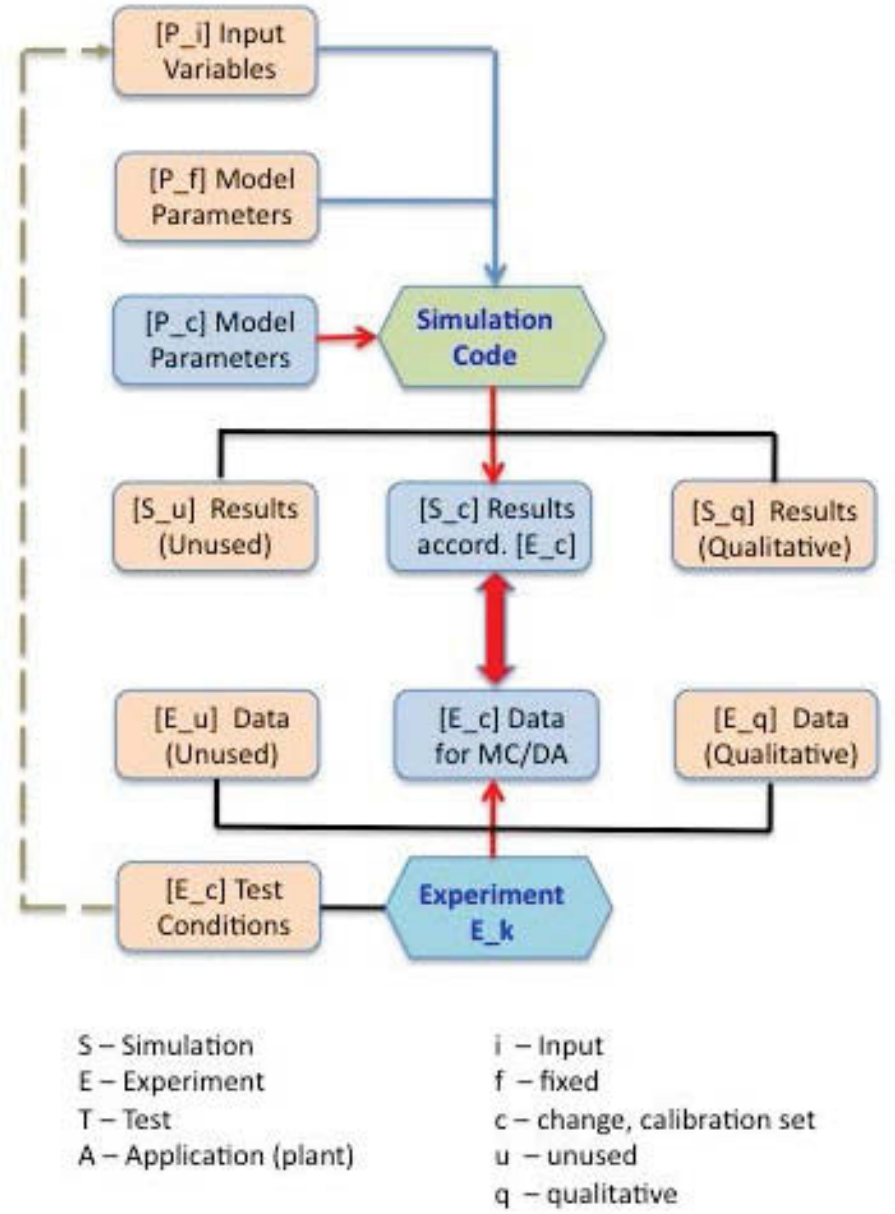

Figure 2.4. The code validation practice. Validation is performed sequentially on individual tests (legacy of code tuning using "viewgraph norm"). This practice of code calibration (parameter tuning) is known to give conflicting results (similar to multiple univariate comparisons). Practice of point/plot-toplot comparison (e.g., for calibration of parameter set [P-c]) uses only a small portion of information [S_c] and [E_c] available from simulation and experiment, respectively. A wealth of qualitative results (insights) [S_q] and [E_q], and an abundance of data generated in both simulation and experiment [S_u] and [E_u] remain unused in model calibration and validation.

\section{Recommendation 2.4: Establish a CIPS-specific integrated calibration and validation plan.}




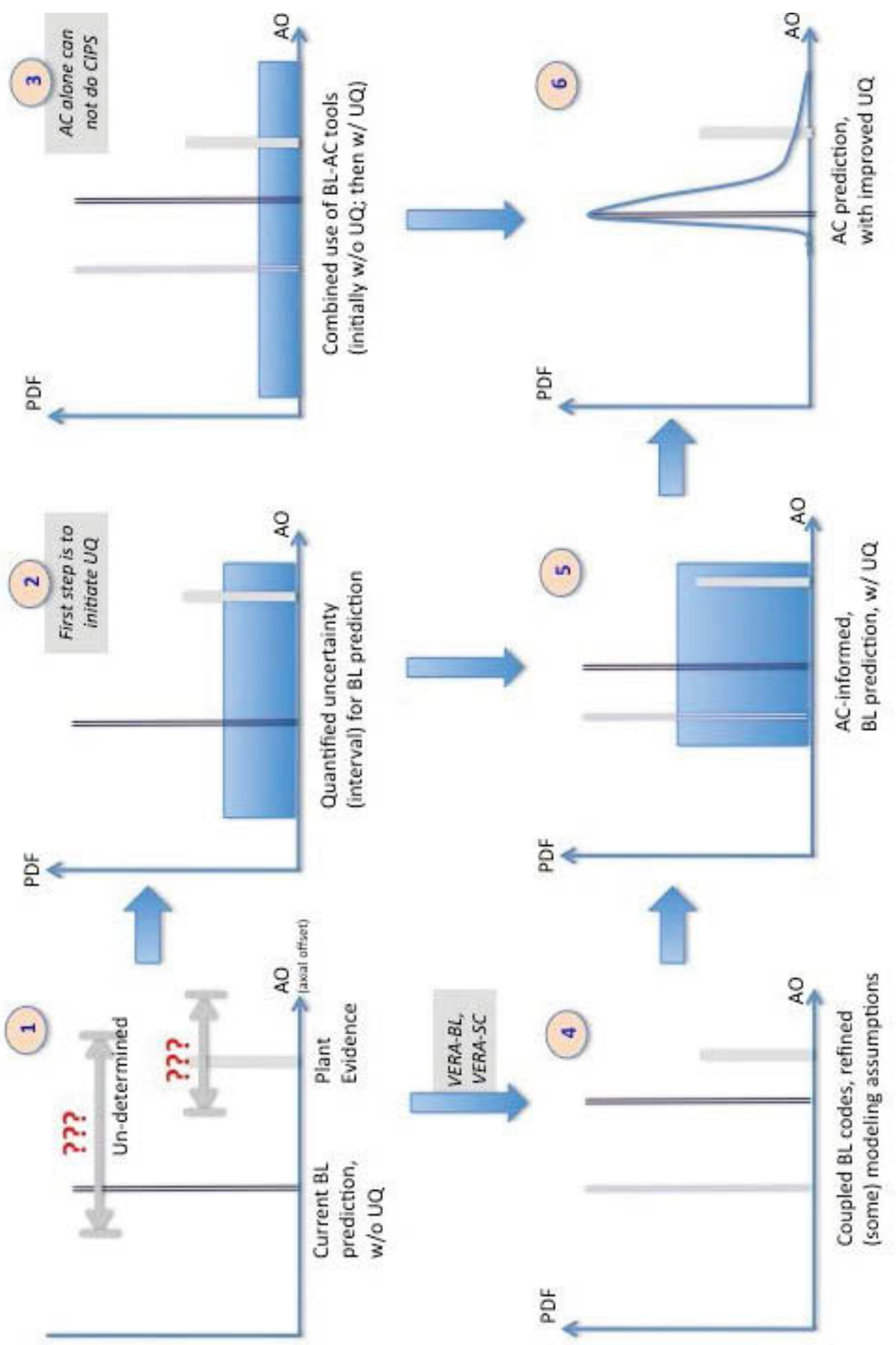

Figure 2.5. Quantitative assessment of calculated "quantity of interest" (QOI), e.g. Core Power Axial Offset in CIPS. 


\subsection{Validation Data and Database Support}

\subsubsection{Overview}

Across CASL, the following groups deal with one or another aspect of validation and validation data, that have a relationship to the CIPS challenge problem solution.

AMA Focus Area:

- Validation Plan

- Core neutronics validation

- Thermal-hydraulics validation

- CRUD (challenge problem) validation

\section{THM Focus Area:}

- Tests and benchmarks: single-phase CFD

- Test and benchmarks: two-phase CFD

- Validation experiments: bubble dynamics, subcooled flow boiling

\section{RTM Focus Area:}

- Progression benchmarks

\section{MPO Focus Area:}

- MAMBA (code assessment/validation plan)

\section{VUQ Focus Area:}

- Cross-Focus Area Validation Data Committee (overall policy, process and infrastructure development)

Presently, working on specific technical topics (core neutronics, thermalhydraulics, fuels, materials) CASL researchers, both individually and as focus teams, own and/or have access to a wealth of data in their respective expertise areas including international databases (e.g., IAEA/OECD International Fuel Performance Experiments IFPE Database; IAEA International Reactor Physics Benchmark Experiments IRPhBE database, and ORNL-hosted Generation IV Materials Handbook database). In addition, under appropriate arrangement, special-purpose and proprietary data from plant measurements (e.g., Voggle's crud data), in-pile experiments (e.g., Halden Reactor Project's AOA tests), out-of-pile experiments (e.g., Westinghouse Advanced Loop Testing) as well as more fundamental data from separate-effect experiments (e.g., MIT, TAMU) are made available for use in CASL validation tasks.

Currently, the data are scattered in the hands of subject matter experts (SME), residing within technical groups within different Focus Areas. An 
effort was initiated to survey the data, to develop a common procedure for data classification and qualification, and to define and develop a CASL-wide infrastructure for hosting databases that meet a wide range of requirements (e.g., secure and convenient web-based access to data with different proprietary classes, compatibility with VUQ methods and tools). This work is to be supported by the CASL Validation Data Committee (Working Group), with participation of researchers from all Focus Areas.

\section{Recommendation 2.5: Establish a CASL-wide policy, process and infrastructure on validation data management, including inventory, warehousing, processing, and controlled secure access.}

Table 2.3. Outcome of validation data review.

(SET: Separate-Effect Test; IET: Integral-Effect Test, PMO: Plant Measurements and Observations, including PIE).

\begin{tabular}{|c|c|c|c|c|}
\hline & & Relevance & Scaling & Uncertainty \\
\hline PMO & $\begin{array}{l}\text { International Fuel Performance Experiments IPFE } \\
\text { Recommendation: Low-to-medium priority }\end{array}$ & $\begin{array}{l}\text { CRUD: } 2 \\
\text { GTRF:2 } \\
\text { PCI: } 3\end{array}$ & $\begin{array}{l}\text { CRUD: } 1 \\
\text { GTRF:1 } \\
\text { PCl: } 3\end{array}$ & $\begin{array}{l}\text { CRUD: } 0 \\
\text { GTRF:0 } \\
\text { PCl: } 2\end{array}$ \\
\hline PMO & $\begin{array}{l}\text { Data collected from operational plants } \\
\text { Recommendation: High priority }\end{array}$ & $\begin{array}{l}\text { CRUD: } 4 \\
\text { GTRF: TBI }\end{array}$ & $\begin{array}{l}\text { CRUD: } 4 \\
\text { GTRF: TBI }\end{array}$ & $\begin{array}{l}\text { CRUD: } 0-2 \\
\text { GTRF:0-1 }\end{array}$ \\
\hline $\begin{array}{l}\text { In-pile } \\
\text { IET }\end{array}$ & $\begin{array}{l}\text { Studsvik Cladding Integrity Project SCIP } \\
\text { Recommendation: High priority }\end{array}$ & $\begin{array}{l}\text { CRUD: } 2 \\
\text { GTRF:3 } \\
\text { PCI: } 4\end{array}$ & $\begin{array}{l}\text { CRUD: } 2 \\
\text { GTRF:2 } \\
\text { PCl: } 4\end{array}$ & $\begin{array}{l}\text { CRUD: } 0 \\
\text { GTRF:0 } \\
\text { PCl: } 2\end{array}$ \\
\hline $\begin{array}{l}\text { In-Pile } \\
\text { IET }\end{array}$ & $\begin{array}{l}\text { Halden Reactor Project Axial Offset Anomaly AOA Tests } \\
\text { Recommendation: High priority }\end{array}$ & $\begin{array}{l}\text { CRUD: } 3 \\
\mathrm{PCl}: 2\end{array}$ & $\begin{array}{l}\text { CRUD: } 2 \\
\text { PCI: } 1\end{array}$ & $\begin{array}{l}\text { CRUD: } 2 \\
\text { PCl: } 1\end{array}$ \\
\hline $\begin{array}{l}\text { Out-of- } \\
\text { pile IET }\end{array}$ & $\begin{array}{l}\text { Westinghouse Advanced Loop Tester WALT } \\
\text { Recommendation: High priority }\end{array}$ & $\begin{array}{l}\text { CRUD: } 4 \\
\text { DNB: } 3\end{array}$ & $\begin{array}{l}\text { CRUD: } 2 \\
\text { DNB: } 3\end{array}$ & $\begin{array}{l}\text { CRUD: TDB } \\
\text { DNB: TBD }\end{array}$ \\
\hline $\begin{array}{l}\text { Out-of- } \\
\text { pile IET }\end{array}$ & $\begin{array}{l}\text { New Experimental Studies of Thermal-Hydraulics of Rod Bundles NESTOR } \\
\text { Recommendation: High priority }\end{array}$ & $\begin{array}{l}\text { CRUD: } 3 \\
\text { DNB: } 3\end{array}$ & $\begin{array}{l}\text { CRUD: } 2 \\
\text { DNB: } 2\end{array}$ & $\begin{array}{l}\text { CRUD: TDB } \\
\text { DNB: TBD }\end{array}$ \\
\hline SET & $\begin{array}{l}\text { Rod Bundle Heat Transfer RBHT : PSBT / BFBT (for PWR focus in Phase 1) } \\
\text { Recommendation: High priority PSBT }\end{array}$ & $\begin{array}{l}\text { CRUD: } 3 / 1 \\
\text { GTRF: } 3 / 1 \\
\text { DNB: } 4 / 1\end{array}$ & $\begin{array}{l}\text { CRUD: } 3 / 2 \\
\text { GTRF: } 3 / 2 \\
\text { DNB: } 3 / 2\end{array}$ & $\begin{array}{l}\text { CRUD: } 3 / 3 \\
\text { GTRF: } 3 / 3 \\
\text { DNB: } 3 / 3\end{array}$ \\
\hline SET & $\begin{array}{l}\text { Subcooled flow boiling SFB: covering a range of SETs on participating } \\
\text { phenomena (over } 20 \text { data sets) } \\
\text { Recommendation: New, well-controlled, VUQ-grade SETs on SFB }\end{array}$ & $\begin{array}{l}\text { CRD/GTRF/ } \\
\text { DNB: range } \\
{[2-4]}\end{array}$ & $\begin{array}{l}1 \text { (low } \\
\text { pressure) } \\
3 \text { (high P tests) }\end{array}$ & In range [1-2] \\
\hline SET & $\begin{array}{l}\text { Vibration Investigation of Small-scale Test Assemblies VISTA } \\
\text { Fuel Assembly Compatibility Test System FACTS }\end{array}$ & GTRF: 4 & GTRF: 4 & GTRF: TBD \\
\hline
\end{tabular}

An overview of data sources and their characteristics is provided in " $C A S L$ Validation Data: An Initial Review" (Dinh, 2011). The review identified potential issues for CASL associated with deficient data quality for VUQ study, data heterogeneity, long acquisition time, and high risk of new, VUQgrade experiments. As an initial step toward quantification of epistemic uncertainty, a grade system (from 0 to 4 ) is used to characterize data by their 
(experiments) Relevance, Scaling, and Uncertainty (R/S/U). A summary of findings and recommendations is provided in Table 2.3.

In Sections 2.5.2-2.5.6, CIPS-related validation activities pursued and planned in CASL are discussed for five technical capability areas, along with their respective modeling requirements and approaches.

\subsubsection{Radiation Transport and Reactor Physics}

This area has been most established in CASL to start with. The requirement to predict pin power across the core accurately stems from the very nature of CIPS as a "reactor physics/core power anomaly" phenomenon. Further, the current lack of understanding of mechanisms by which state-ofthe-practice methods for CIPS analysis fall short in predicting CIPS QOIs stipulates hypotheses that certain fine-scale phenomena and/or multi-physics interactions at those scales are not properly captured in coarse-grain and loosely-coupled analysis methods.

An AMA-led "Neutronic Validation" task (Zhang, 2012) reviewed databases of experiments on radiation transport and reactor physics. They identified several databases of general interest ${ }^{6}$. However, it is noted that tests included in theses databases were performed for conditions that incorporated boron/crud effect.

A set (currently, 10) of progression benchmarks was defined to help focus and assess the development of advanced radiation transport capability for VERA-AC. At this time, the development targets a generic capability.

The first five benchmarks, \#1-5, include no thermal-hydraulic or fuel temperature feedback, performed for a $3 \times 3$ and $17 \times 17$ configuration of a PWR fuel assembly.

The second five benchmarks, \#6-10, include increasingly more complex effects, e.g., thermal-hydraulic feedback, fuel temperature feedback, xenon poisoning, fuel depletion and fuel shuffling.

6 "Neutronic Validation", Zhang (March, 2012):

Strawbridge-Barry: 101 Criticals

B \& W Critical Experiments with Urania-Gadolinia: 9 configurations International Criticality Safety Benchmark Evaluation Project (ICSBEP): 4405 criticals

International Reactor Physics Evaluation Project (IRPhEP):

13 cases from 9 different reactors 
The comments below apply to the benchmark plan as formulated presently (CASL-2011-0074-000-CI).

Comment \#RT.1: The choice of test configuration and scope of benchmarks provide a sterilized platform for executing and demonstrating the simulation capability as it evolves. From a VUQ standpoint, such a platform is useful e.g., in enabling verification study and sensitivity analysis. However, no actual data from experiments or plant measurements are involved. As a result, the VUQ plan based on benchmarks falls short of being a technical basis for quantitative assessment and validation of the capability as required in CIPS challenge problem applications.

Comment \#RT.2: Since the benchmarks by themselves are not enough to determine applicability of the models and model parameters used, a suite of validation tests is required in conjunction with the benchmarks. The guiding principle in implementing the validation suite is that the select tests help identify and quantify uncertainty in models and model parameters that dominate the predicted QOI's uncertainty. Given the history of reactor physics, the radiation transport validation plan must balance between basic tests and CIPS-relevant tests, with the goal to capture/bound/determine uncertainties in CIPS scenarios.

Comment \#RT.3: The development and benchmark plan for reactor physics is to be extended beyond the first ten benchmarks to include CIPS-related reactor physics phenomena. Specifically, this includes the effect of boron feedback, both soluble boron in coolant and boron content in crud regions. The capability requires generation of cross sections under various configurations with presence of crud whose compositions include boron and other chemicals e.g., additives and corrosion products.

Comment \#RT.4: With focus on the CIPS challenge problem objective (predicting AOA over the burnup cycle with uncertainty less than 3\%) and given computational burden of VERA-AC (RT) codes, a multi-scale integration is a must (Figure 2.6). Initially rudimentary cross-scale integration scheme is to be refined in time (homogenization, conditioning, crossscale/cross-code uncertainty propagation, error control) and optimized for efficiency and accuracy.

Recommendation 2.6: Develop "gap-tooth" scheme for integration of VERA-BL (e.g., neuron diffusion) capability for long-transient simulation and $V E R A-A C$ (e.g., radiation transport) capability for high-resolution snapshots simulation. 
Comment \#RT.5: There needs to be benchmarks and validation experiments to assess multi-scale integration required for CIPS simulation that is long in time e.g., months (possible with neutron diffusion ND) and accurate in space e.g., pin-resolved (with radiation transport RT).
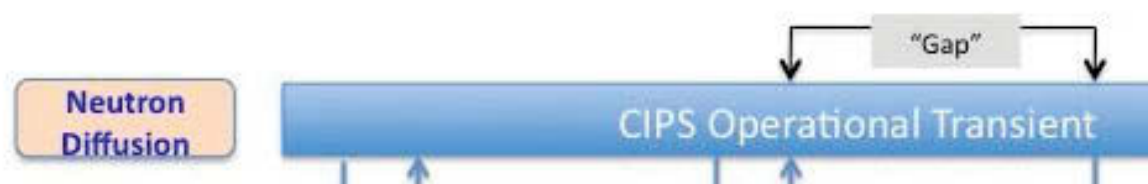

diation

Transport

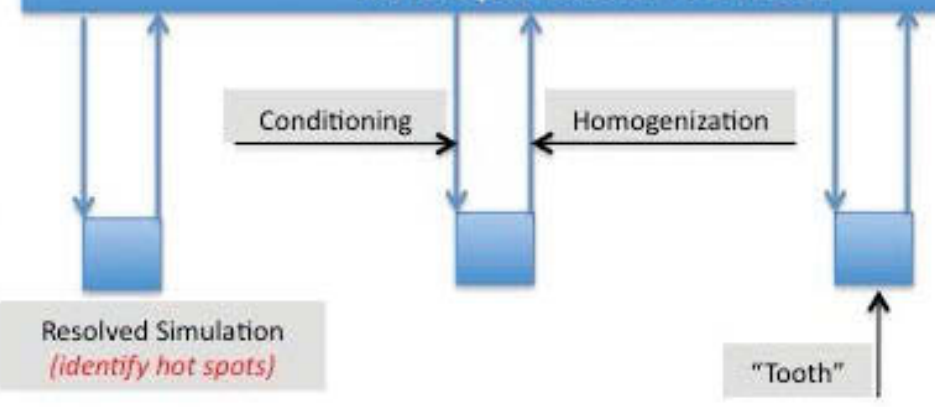

\section{X-sections Boron/crud effect}

Figure 2.6. "Gap-tooth" multi-scale integration scheme, which applies to all physics of importance for CIPS.

\section{Recommendation 2.7: Develop benchmarks for multi-scale ("gap- tooth") integration.}

Comment \#RT.6: Excessive crud deposition in certain scenarios may have been resulted from combination of system/power transients not accounted for in current industry's analysis. Examination of hypotheses about local power anomaly affecting crud growth require capability in treating reactor transients, including control rod movements, asymmetric distribution/influx of coolant, boron and corrosion products. 


\subsubsection{Thermal-hydraulics}

There area wide range of models and simulation codes that are germane to nuclear reactor thermal-hydraulics. While higher-fidelity/higher-resolution models are trendy, engineering applications remain bound to computationally affordable lower-resolution simulations. This is particularly true when the engineering process involves a long time scale (see Figure 2.7). From an engineering practice standpoint, development and integration of higherresolution AMS codes are meaningful when they have the potential for generating results for conditions of interest (PWR, CIPS) that aid uncertainty reduction in coarser-grain models. For the CIPS challenge problem, simulation capability at the three upper levels (coarse-grain models) is considered on the "uncertainty reduction" side (Figure 2.7$)^{7}$.

The highest resolution (lowest length scales) simulation capability of T-H in core fuel assemblies is provided by CFD / CMFD codes in VERA, currently with Star-CCM+ and DREKAR, and in the future with HYDRATH. Development and validation of HYDRA-TH is the focus in THM. The coarse-grain (subchannel) simulation code VIPRE-W (and its equivalent COBRA) is used in VERA-BL for core thermal-hydraulics. System-level simulation is also required in CIPS as to enable simulation of transport of corrosion products over long period. In VERA-BL, this is provided by an embedded function in BOA code. VERA-AC considers possibility to use a system T-H code like RELAP5. The later can provides more accurate modeling of $\mathrm{T}-\mathrm{H}$ in the reactor primary system over a wide range of plant operational and abnormal transients, including e.g., loop-asymmetric scenarios induced by processes in the plant's secondary side.

Relative to T-H capability to inform the CIPS challenge problem, two paths are being pursued in THM (with AMA support):

[P.1] Assessing and improving a commercial code Star-CCM+

- validation of single-phase capability (turbulence models)

- testing and refining two-phase flow capability

[P.2] Developing and assessing an open-source code Hydra-TH

- testing and refining Hydra (single-phase flow) capability

- implementing and testing two-phase flow capability

\footnotetext{
${ }^{7}$ DNS/LES/ITM models for two-phase flow remain strongly dependent on sub-grid-scale (SGS) micro-physical modeling (e.g., dynamics of evaporating thin liquid meniscus, effect of long-range molecular forces, surface nanomorphology and material heterogeneity). The database is deficient for the purpose of qualifying assumptions and calibration of LES/ITM sub-grid-scale (SGS) models under PWR (CIPS) conditions.
} 


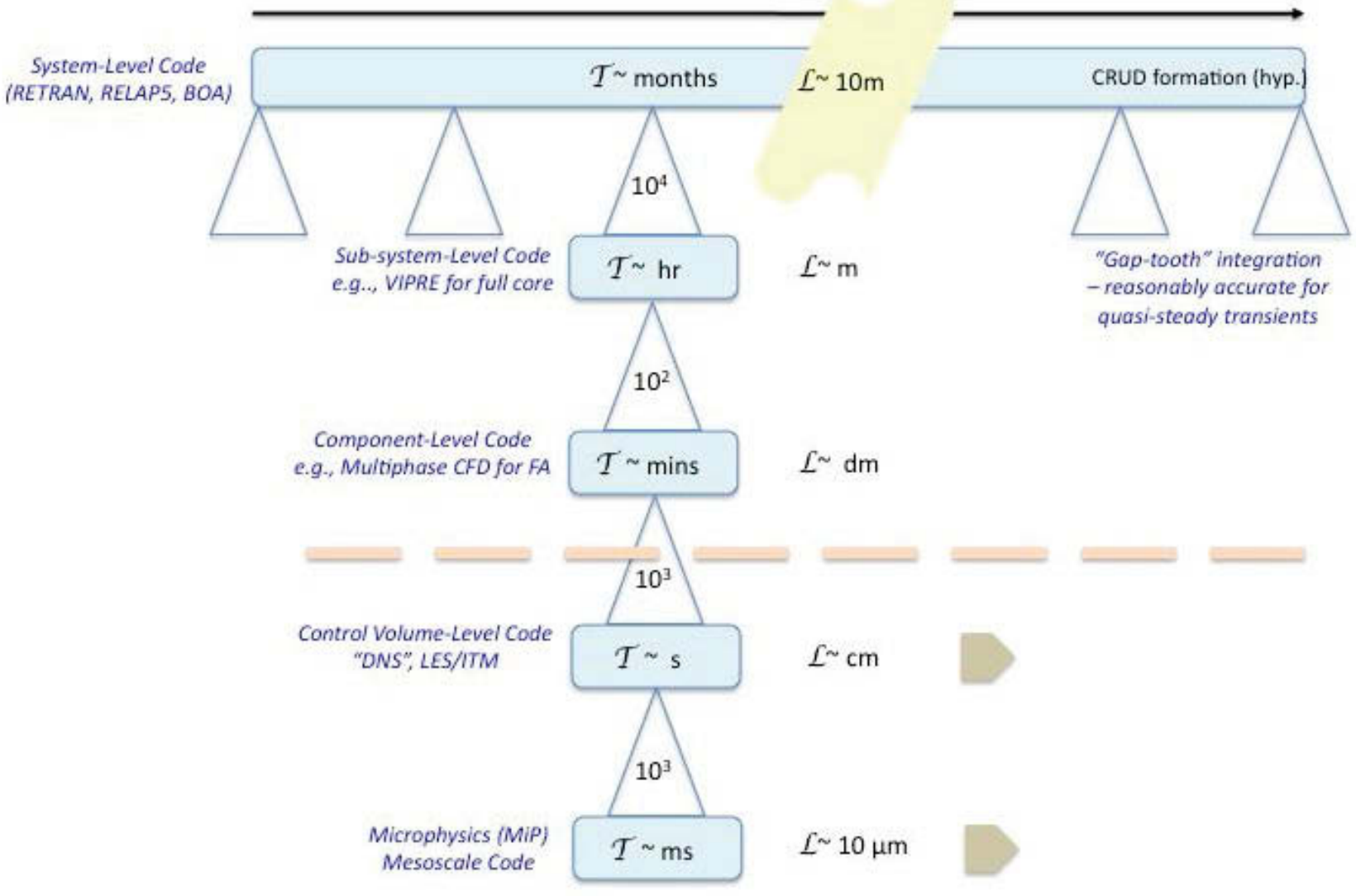

Figure 2.7. Time/length scales and codes in thermal-hydraulics. (DNS: Direct Numerical Simulation; ITM: Interface Tracking Method;LES: Large Eddy Simulation; FA - fuel assembly)

Keeping in mind that an important part of $\mathrm{T}-\mathrm{H}$ simulation is to support crud modeling, there are two ways by which one can apply VERA CFD/CMFD capability to inform VERA-BL models:

\section{Path $[\mathbf{A}]$}

(A.1) Assuming that subcooled boiling is captured by surface overheating

(A.2) Using single-phase CFD simulation to obtain highly-resolve thermal-fluid fields

(A.3) Mining the result for refining coarse-grain subchannel T-H models and providing input to MAMBA, RT

\section{Path [B]}

(B.1) Modeling subcooled flow boiling in CMFD framework

(B.2) Using two-phase CMFD simulation to obtain highly-resolve thermal-fluid fields

(B.3) Mining the result for refining coarse-grain subchannel $\mathrm{T}-\mathrm{H}$ models and providing input to MAMBA, RT 
Comment \#TH.1: Path [A] provides a more robust simulation capability, it is made possible by invoking modeling simplifications that entail substantial model-form uncertainty. Furthermore, the simulation results do not give details about phase density (needed for neutronics calculation) and boiling structures such as bubble meniscus dynamics, dry patches, etc. (needed for near-wall scalar transport for crud calculation).

Comment \#TH.2: Path [B] has potential to provide more detail information about structures and processes in the near-wall region that provide boundary conditions to the CRUD models. However, due to a large number of models and model parameters involved, this path entails large uncertainty and requires research, including advanced experiments and characterizations, to reduce the uncertainty.

\section{Recommendation 2.8: Perform VUQ-grade experiments (two-phase flow, SFB) and analysis to establish impact of assumptions, maturity, and usefulness of Paths [A] and [B].}

A wide range of experimental data are needed to support validation of T-H simulation capability. VIPRE-W (COBRA) and RELAP5 codes have been used in the industry and were subject to extensive assessment and calibration, using a large number of tests. Notably, the assessment / calibration base of VIPRE-W and RELAP5 codes covers a broad range of conditions inclusive of LWR operation, transients, and accidents. As a result, the resulting generic models are not necessarily optimal for CIPS-relevant conditions.

A set of benchmarks for validating VERA-AC thermal-hydraulics simulation capability, specifically the CFD and CMFD (multi-phase CFD) codes, was defined, as follows.

\section{Basic CFD validation ${ }^{8}$ \\ Turbulent pipe flow \\ Axisymmetric sudden expansion \\ U-bend \\ Turbulent natural convection (air cavity) \\ Specific-geometry CFD validation \\ Heated rod bundle \\ $5 \times 5$ PWR fuel assembly (TAMU)}

\footnotetext{
8 The CFD benchmarks set was developed by THM/AMA (Pannala \& Stagg, Nov. 2011).
} 


\section{Basic CMFD validation ${ }^{9}$ \\ CFD-P1: Pipe. Air/water bubbly Flow. (Wang, 1987)}

\section{Subcooled flow boiling (SFB) tests for CMFD validation \\ CFD-P2: Pipe. Axial void and temperature data (Bartolomei, 1967) \\ CFD-P3. Annulus. Radial void, velocity, temperature, kinetic energy \\ (Velindala, 1997) \\ CFD-P4: Pipe. Axial void and temperature data (Rouhani, 1966) \\ CFD-P5: Pipe. Axial/radial void data (St. Pierre, 1965)}

While these benchmarks are valuable on their own right, it remains open how relevant these tests are for the CIPS (reactor prototypic) conditions, and how to interpret, and make use of, the benchmark results (failure or success) relative to the VERA capability to predict CIPS. In other words, the benchmarks set was defined generally and without pointing out how a given test addresses specific model-form and model-parameter uncertainty. Answer to this question helps determine a faction, by which the defined set of benchmarks covers the capability space, given a large number of degrees of freedom in models and codes involved.

Comment \#TH.3: For CMFD validation (CFD.P1... CFDP.5), experiments were performed several decades ago. Experimental "data" exist largely as plots in reports and dissertations. Limited to no information about measurement uncertainty or reproducibility is available.

Comment \#TH.4: Air/water and subcooled flow boiling tests were performed under (system pressure, flow rate, geometry, heater surface, heat flux) conditions far from PWR operating conditions. When boiling (nucleation, wettability) is concerned, non-prototypical surface material/morphology (crudded zirconium vs clean steel) and coolant chemistry (reactor water vs. distilled water) introduce uncertainty that is hard to quantify.

Comment \#TH.5: Measurements in the past tests are integral (as opposed to tests using advanced high-resolution diagnostics). Benchmarking against the old (integral) data provides a basis to assess performance of "composite/integrated models", but it is not an effective means for characterizing performance of modeling assumptions in individual closure relations and sub-grid-scale models.

\footnotetext{
${ }^{9}$ The CMFD benchmarks set was developed by THM (M. Podowski, February, 2012).
} 


\subsubsection{Coolant Chemistry and CRUD}

For the CIPS challenge problem, the quantity of interest is Axial Offset (AO), which is a core physics calculation output. This axial offset depends on characteristics of crud, its mass (observed to be of the order of $10 \mathrm{lbs}$ in a core over a severe CIPS scenario), and composition (primarily the boron content). This is a relatively newer domain in nuclear reactor engineering compared to reactor physics, $\mathrm{T}-\mathrm{H}$, and fuels. It involves thermodynamics of complex chemical aqueous system under high temperatures, high pressure, and microscale multiphase transport in complex geometries (porous layer) with phase change.

For modeling and simulation of coolant chemistry/CRUD processes, CASL employs industry's code BOA in VERA-BL suite and research code MAMBA in VERA-AC. Sources of experimental data identified for model assessment/UQ for VERA's coolant chemistry and CRUD simulation capability include

- Out-of-pile tests

$\circ \quad$ Westinghouse Advanced Loop Testing (WALT)

- Single rod, synthetic crud (EPRI TR 1022896)

- In-pile tests (HRP-AOA tests)

O Halden Reactor Project - AOA test series: A test program (in three cycles) sponsored by the US DOE and EPRI.

- Plant measurements and observations

○ Crud data from Vogtle and Seabrook plants

\footnotetext{
${ }^{10}$ Bennet et al. (2004, 2007): "Series 1: A bundle of eight tests rods (active fuel length $60 \mathrm{~cm}$ ) was irradiated in the Halden reactor for 349 full power days (3 reactor cycles) under PWR water chemistry and thermal-hydraulic conditions. One of the test requirements was that no boiling should occur long the lower section of the fuel rods and that sub-cooled nucleate boiling was required along the upper section. Hence, the lower $(20 \mathrm{~cm})$ and upper $(40 \mathrm{~cm})$ sections of the test rods were fuelled with $\mathrm{UO}_{2}$ with different enrichments. On-line, in-core instrumentation included a diameter gauge to detect crud deposition, and neutron detectors and coolant thermocouples to detect flux/power depressions. An Fe-Ni-EDTA solution was added to the loop coolant to accelerate crud deposition. After irradiation, crud samples were collected for post irradiation exposure to investigate their composition and morphology.

Series 2: A typical experiment contains from one to six test fuel rods, with an active fuel length of up to $60 \mathrm{~cm}$. PWR water chemistry is simulated by additions of $\mathrm{LiOH}$, $\mathrm{B}(\mathrm{OH}) 3$ and dissolved hydrogen, together with any required additives, for example zinc. Although the coolant flowrate through the test section (1 to $1.8 \mathrm{~m} / \mathrm{s})$ is a factor of three lower than that in a commercial PWR, a representative degree of sub-cooled nucleate boiling (SNB) can be achieved by adjustment of the coolant inlet temperature and fuel rod power."
} 
Comment \#CC.1: The EPRI's BOA code can be made to couple with MAMBA code, so to provide necessary boundary conditions to execute the MAMBA models. It is noted that the BOA code uses a simplified treatment of thermal-hydraulics. Consequently, processes that govern transport of soluble chemicals (boron, additives) and particulate materials (corrosion products) in the reactor core, in fuel assemblies, sub-channels, and toward cladding surface are necessarily treated with significant approximations.

Comment \#CC.2: While detailed sensitivity/uncertainty analysis is to be carried out, over-simplified treatment of operational factors can be a major source of (model-form) uncertainty. This can include assessment of system's corrosion products inventory, efficacy of crud removal campaigns, and other plant operational controls. A quasi-steady treatment using time-averaged "nominal" corrosion and corrosion products influx would miss capturing a flush of corrosion products release during a plant transient (e.g., due to an event in the secondary side that causes transient response in a steam generator). Such scenarios, or rather, their inaccurate treatment, may have been responsible for the unpredictability of crud-related behaviors where excessive cruds were found in some plants, under some burnup cycles, and only in some fuel assemblies. The above discussion is to reiterate the critical importance of a system and systematic approach in uncertainty reduction.

Comment \#CC.3: Within MAMBA code, there is a large number of mechanistic and empirical models involved. Modeling assumptions used, however reasonable they may appear, are subject to limitations, and often are not applicable for conditions of interest. To facilitate future work in identification and quantification of model-form and model-parameter uncertainties, each and every model assumption must be documented and their software implementation must allow access to it by VUQ tools.

Comment \#CC.4: The number of models and model parameters in MAMBA is far larger than the limited number of experiments (WALT, HRP) and plant measurements that are available for analysis. Notably, the measurements performed in these tests are integral (accumulative in time), and not all test/plant conditions needed for modeling were acquired or available, rendering significant difficulty in using them for code / model assessment and UQ. 


\subsubsection{Nuclear Fuels and Claddings}

Nuclear fuels and claddings system (including thermo-mechanics, fission product distribution, and heat transfer) are tightly coupled with core neutronics and influence core thermal-hydraulics. For the CIPS challenge problem, it is expected that fuels/claddings be treated consistently with core neutronics and thermal-hydraulics, namely it is implemented in two levels:

- Coarse-grained simulation - FRAPCON/FRAPTRAN/FALCON class for VERA-BL

- Three-dimensional, pellet-resolved simulation (to be coupled with RT) - Peregrine/Bison for VERA-AC

Fuels/claddings involve complex processes, which are described in these codes through a number of models (listed below). Each process model includes modeling assumptions and model parameters that must be assessed and calibrated. As the fuel burns up, the participating processes are known to vary with operating conditions over the in-core life cycle.

\section{Nuclear Fuel:}

Swelling and densification

Thermal and irradiation creep

Pellet fracture

Fission gas production and release (incl. poison)

Oxygen transport, microstructure evolution

Thermal, structural properties (function of burnup, temperature)

\section{Cladding:}

Thermal and irradiation creep

Irradiation growth

Thermal, structural properties

\section{Gap/Plenum:}

Gap heat transfer

Pellet-cladding mechanical contact

Gap gas/mixture thermodynamics

In a previous study (Dinh, 2011), several databases of potential relevance to validation of Fuels/ Cladding model and CIPS AOA simulation were identified. ${ }^{11}$

${ }^{11}$ OECD-NEA International Fuel Performance Experiments (IFPE) Database

Studsvik Cladding Integrity Project (SCIP)

Halden Reactor Project (HRP) 
Presently, the validation plan for Peregrine/Bison is still under development. An initial assessment of the Bison code was performed against a Halden Reactor Project test IFA-432 (Wiliamson et al., 2012). While an indepth analysis and systematic approach are needed for developing the validation plan for fuels/claddings codes like Peregrine/Bison, scoping considerations suggest that the Halden Reactor Project program has created a large number of tests (series IFA; see examples listed below) that serve as technical basis for comprehensive assessment and calibration of Fuels/Claddings simulation codes. In addition, series HR-AOA provides data obtained under conditions that simulate CIPS.

\section{Generic LWR fuel modeling capability assessment}

IFA-629/700/720 - integrated fuel performance

IFA-650/655 - fuel response to transient

IFA-681 - fuel high burnup (w/ Gd doping)

IFA-699 - cladding creep

IFA-629/701 - fuel creep

IFA-708 - cladding corrosion/water chemistry

IFA-677/716 - fission gas release

\section{CIPS-related fuel/cladding}

HR-AOA test series 


\subsubsection{Multi-physics Simulation}

CIPS is inherently a multi-physics problem, and this marks its most significant departure from a single-physics problem like CFD or radiation transport. As discussed in previous sections, key physics in CIPS are radiation transport/core neutronics (denoted as RT), thermal-hydraulics (TH), coolant chemistry and CRUD (CC) and Fuels. The CASL strategy to CIPS is to develop and assess multi-physics simulation capability, both VERA-BL and VERA-AC.

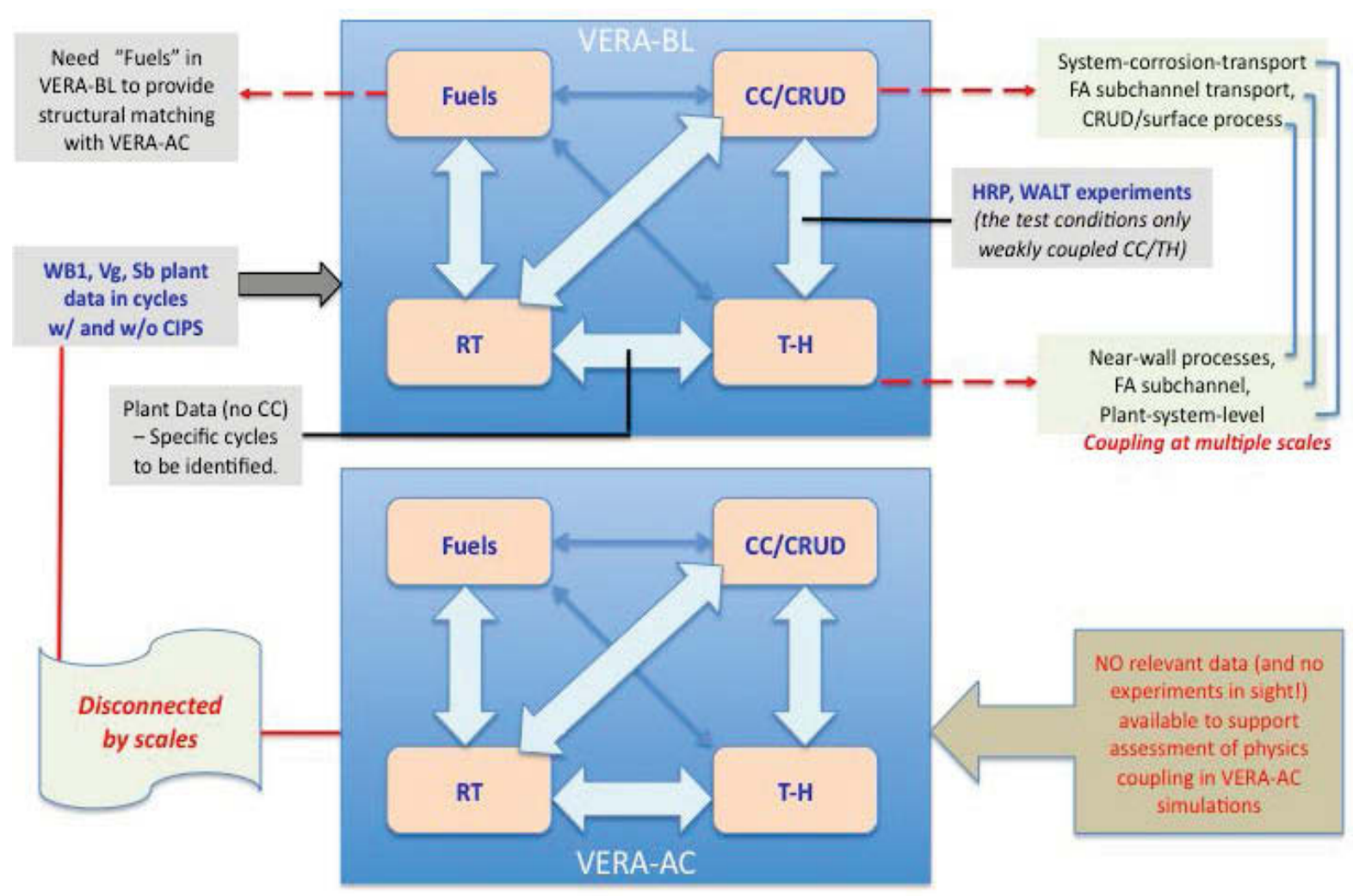

Figure 2.8. Physics components and multi-physics interactions. Shown for two scales, respectively described by VERA-BL and VERA-AC codes. Lines relate potential sources of validation data. Mapping available experiments and plant data to the validation of multi-physics capability shows the dramatic paucity of validation base. Even for $T / H-C C$ and $R T-T / H$ couplings, where some experiments and plant data are identified, the data quality is such that they are expected to have a limited value in characterizing the coupling. The number of interface models (estimated to be in the order of 10 to 30) and model parameters (estimated to be in the range of 30 to 100) needed to be calibrated in a CIPS multi-physics problem is too large for a limited number of available tests and the scope of the test data. 
Note that all physics simulation capability brought into VERA or developed in CASL for VERA have been developed under a "divide-andconquer" paradigm (i.e., they are "traditional" single-physics codes with provision for input from other physics through constitutive laws [in form of source terms, physical properties]). In certain cases, the inter-physics exchanges are fairly straightforward, particularly when the governing model for one physics was derived with the other physics in mind (e.g., between neutronics and $\mathrm{T}-\mathrm{H})$.

However, in emerging applications (e.g., coolant chemistry/CRUD and thermal-hydraulics), the (e.g., two-phase flow) model and resulting (T-H) code developed previously for another "traditional" objective do not have provision for the effect of the other new physics with different quantities of interest (e.g., crud-related near-wall transport of chemicals and particulates in coolant). This necessitates additional, sometime significant, work on modeling both sides to enable the coupling to happen ${ }^{12}$.

Furthermore, due to different characters of mathematical models (equations) that govern the participating physics, their optimal numerical solution called for using different discretization schemes with differing meshing requirements. Communication between different physics solutions requires matching of their parameters through homogenization/redistribution of individual physics. Consequently, the "divide-and-conquer" with subsequent integration strategy has been known to impose limitations on numerical implementation (e.g., time stepping) and/or entail numerical errors. The limitations most severe in systems where participating physics are nonlinearly coupled ${ }^{13}$.

Thus, multi-physics simulation necessarily involves a range of assumptions in modeling and numerical treatment. Implementation of validation hierarchy and quantification of uncertainty of the predicted QOI (AO in CIPS) requires validation of pair-physics coupling, and trio-coupling, and full-set coupling. This also assumes that individual physics have been assessed.

\footnotetext{
${ }^{12}$ See Chapter 4 for a more detailed discussion on TH-CC interface modeling.

${ }^{13}$ Certain couplings may not appear restrictive in a short time transient, but become such in high-resolution simulation of long transients, when substantial time intervals (e.g., between snapshot solutions) are necessitated. This recognition of solution/simulation time scale is instrumental in putting into perspectives the debate about strong vs. light couplings.
} 
Table 2.4 provides an overview of CASL-VERA capability related to CIPS prediction. The right column identifies potential sources of data that can be used to assess the multi-physics coupled capability. This second review confirms a previous finding of (Dinh, 2011) that a substantial gap exists in data for supporting validation of VERA multi-physics simulation capability.

Table 2.4. CIPS-related multi-physics simulation and validation data sources.

\begin{tabular}{|c|c|c|c|}
\hline & $\begin{array}{l}\text { Degree of physics } \\
\text { coupling in CIPS }\end{array}$ & Capability set [Maturity of Coupled Capability] & $\begin{array}{l}\text { Experiments / Data Sources for Model } \\
\text { Calibration and UQ }\end{array}$ \\
\hline $\mathrm{RT}-\mathrm{CC}$ & Strong & $\begin{array}{l}\text { Denovo-MAMBA [Low]. Can be used only for } \\
\text { snapshot calculations (due to computing costs). Not } \\
\text { commensurate with crud data (long residence time) } \\
\text { ANC9/BOA [High] }\end{array}$ & $\begin{array}{l}\text { None decided. No "snapshot" data identified. } \\
\text { For long transient, HRP AOA (crud!) test is a lead } \\
\text { candidate, with uncertainty on RT side. } \\
\text { As HRP data were used in tuning BOA models, } \\
\text { they are of low value for testing RT-CC coupling. }\end{array}$ \\
\hline RT-Fuels & Strong & $\begin{array}{l}\text { Denovo-Peregrine [Low]. Snapshot calculations. Not } \\
\text { commensurate with fuel performance data. }\end{array}$ & None decided. \\
\hline $\mathrm{RT}-\mathrm{T} / \mathrm{H}$ & Strong & $\begin{array}{l}\text { DeCART/Star (High] } \\
\text { Denovo-Drekar [Low] only for single-phase } T / H \\
\text { Denovo-Hydra-TH [Low] }\end{array}$ & $\begin{array}{l}\text { No experiments identified. Test cases (e.g., } 3 \times 3 \\
17 \times 17 \text { pin models, or larger) are useful, but fall } \\
\text { short of needs in model calibration and UQ. }\end{array}$ \\
\hline $\mathrm{T} / \mathrm{H}$ - Fuels & Medium & Hydra-TH-Peregrine & None identified. (low need or CIPS) \\
\hline $\mathrm{T} / \mathrm{H}-\mathrm{CC}$ & Strong & $\begin{array}{l}\text { Hydra-TH-MAMBA (W/ BOA) [Low]. Key uncertainty is } \\
\text { in T-H/MPO models. Not captured in integral tests. } \\
\text { VIPRE-W/BOA (w/MPO models) [High (Med.)] }\end{array}$ & $\begin{array}{l}\text { HRP Crud tests } \\
\text { WALT experiments }\end{array}$ \\
\hline $\mathrm{CC}$-Fuels & Weak & MAMBA-Peregrine [Low] & $\begin{array}{l}\text { None identified. (low need or CIPS, but can } \\
\text { become important in CILC, PCI). }\end{array}$ \\
\hline $\mathrm{RT}-\mathrm{T} / \mathrm{H}-\mathrm{CC}$ & Strong & $\begin{array}{l}\text { Denovo-Hydra-TH-MAMBA [Low] with } \mathrm{T} / \mathrm{H} \text { and } \\
\text { MAMBA-T/H connection being limiting factors). }\end{array}$ & HRP Crud tests (minimal test of RT) \\
\hline $\begin{array}{l}\text { RT-T/H-CC- } \\
\text { Fueis }\end{array}$ & Strong & $\begin{array}{l}\text { Denovo-Hydra-TH-MAMBA-Peregrine [Low]. See } \\
\text { above. Snapshots only. } \\
\text { ANC9/VIPRE-W/BOA [High] }\end{array}$ & $\begin{array}{l}\text { Plant data from WB1, Vogtle and Seabrook } \\
\text { (Cycles with no CIPS and Cycle with CIPS) }\end{array}$ \\
\hline \multicolumn{4}{|c|}{ Extended couplings inherit issues from subsidiary component couplings. } \\
\hline
\end{tabular}

HRP- Halden Reactor Project; WALT- Westinghouse Advanced Loop Testing; WB1- Watts Bar 1

VERA-AC tools are computationally demanding, hence they are limited to "snapshot calculations", not consistent with the (limited) body of CIPS-related long-transient data, namely crud buildup, fuel burnup, depletion and axial offsets. The tests available, from plants (Vogtle), in-pile (HRP) and integral tests (WALT) were designed to investigate crud buildup, not for testing the multi-physics simulations. Although the setting was inherently multi-physics, certain physics were "silent" or not characterized, making the tests deficient for multi-physics method validation. For example, in-pile HRP tests are not 
amenable for testing reactor physics/radiation transport component. WALT tests are not amenable for testing HYDRA-TH/MAMBA coupling even though flow, boiling and crud deposition are present. Length and time scales over which measurements were made are far (several decades) larger than the scales for which mechanisms of physics coupling are modeled. For examples, coupling between MAMBA and HYDRA-TH occurs through models in boiling nucleation, meniscus evaporation, near-wall (micro-layer) transport of chemicals and particulates (corrosion products). The HRP and WALT experiments offer no data that could be used to assess and calibrate these cross-physics models.

\subsubsection{Cross-cutting Issues}

Comment \#CI.1 (integration framework): The preceding analysis of four technical areas (RT, TH, CC, and Fuels) and their multi-physics interactions points to an overarching issue of (lack of) integration between VERA-AC (high-resolution/high-fidelity) simulation capability and VERA-BL (coarsegrain) simulation capability ${ }^{14}$.

As driven by the mission of using high-performance computing to deliver high-resolution simulations (still snapshots), the requirements currently defined for VERA, and subsequently the development activity are focused on VERA-AC tools, with minor attention on the integration with coarse-grain simulation tools.

Comment \#CI.2 (uniformity in VVUQ): Assessment (validation) of the predictive capability for an (multi-physics/multi-scale) application requires broad range of data and a consistent treatment of uncertainty across disciplines (physics) and scales. To tackle problems like CIPS, the "hub" brings to bear a blend of disciplines with vastly different cultures and practices in VUQ. Even in one area, experts working at different scales or different regimes (of notionally the same physics) carry with them specific rules and tools. The difference can be significant, for example in $\mathrm{TH}$, between single-phase CFD, two-phase/boiling, and ITM communities ${ }^{15}$.

\footnotetext{
${ }^{14}$ VERA-BL is used here to denote the class of capability for predicting CIPS QOIs over long operational transients. Notionally, it is not restricted to industry suite of codes. Practically, due to the project time/resources constraints, the coarse-grain simulation tools are the industry's state-of-the-practice tools.

15 On the other hand, "collocation" of disciplines/expertise/cultures enables crossfertilization of knowledge, creating opportunity for discovering blind spots. Such moments are invaluable for model development and assessment.
} 
Comment \#CI.3 (validation plan): Review of validation activity across CIPS technical areas shows largely ad hoc character, particularly in areas $(\mathrm{TH}$, CRUD, multi-physics), where capability is less mature.

Validation plan exists at high level, aiming at basic functions, and yet to be tailored to specific needs of challenge problems (CIPS in this case). Validation plan and validation metric are still novel concepts, discussed in VUQ literature and yet to be adopted in CIPS-related technical areas.

In case when a range of codes used or under development, validation plan does not call out which codes or specific capability is tested.

Comment \#CI.4 (validation metric): Largely unspoken remain questions: How to interpret diverging outcomes/failures/successes of the tests? What does a particular benchmark outcome say about (or how it is to be factored in assessment of) maturity of the benchmarked predictive capability for reactor applications (conditions)?

Other questions related to the validation metric are: How many models and model parameters (uncertainties) exist in the codes, and what fraction of them is subject to evaluation in the select tests? For the challenge problem, what are the ranges of conditions, and how well do the tests cover these ranges? In other words, whether/to what extent do the select tests/benchmarks identify model-form uncertainty and evaluate model-parameter uncertainty?

\section{Recommendation 2.9: Create a VERA-wide list of models, modeling assumptions, and model parameters. Each validation task must document models/assumptions/parameters scrutinized in the benchmark and additional assumptions made in order to implement the benchmarks. ${ }^{16}$}

Comment \#CI.5 (validation database): Several characteristics of importance to VVUQ are found common across legacy databases and records from past experiments. First, they are specialized data archives, hard to search and not amenable to modern data analytics (data mining). There are no meta data or meta data are not standardized. Second, knowledge about methods for data production, and other tacit information about data are more often than not absent. Third, quality of data is generally poor and deficient for quantification of uncertainty. There is often no way to maintain quality control. Examples are many critical CMFD benchmark data are digitized from plots from reports or journal papers, with high potential for errors in transactions.

\footnotetext{
${ }^{16}$ [including delineation of situations when added assumptions become invalid in applications].
} 
Comment \#CI.6 (validation data): Validation data identified so far in various benchmark activities are (in majority) not CIPS-specific, integral in time and/or space (particularly in TH, and CRUD) and hence not suitable for assessment of individual micro- and meso-scale models and closure relations. Also, there is virtually no quality data to support multi-physics simulation capability assessment.

\subsection{Programmatic Targets and Constraints}

The leading programmatic target in the CIPS Challenge Problem is to provide the AMS (VERA) capability to analysts (represented by AMA experts) that enable them to achieve "prediction of CIPS AO with uncertainty less than 3\%", by the end of CASL Phase I. The AMA-led planning document breaks this target into a set of surrogate milestones, whose demonstration effectively mean mission accomplishment.

For CIPS, current high-level AMA-oriented milestones are (Gehin, 2011)

VERA-BL tools:

- Run coupled tools (fine mesh) and compare to data

- $\quad$ Perform UQ for boron and crud

- Add improved MPO (MAMBA) models to BOA and compare coupled tools to data ${ }^{17}$

- $\quad$ Perform final UQ for coupled tools

- Implement improved coupled tools for risk assessment

VERA-AC tools:

- Run coupled CFD/Neutronics/BOA for WB, 3 cycles

- Run coupled CFD/Neutronics tools for 3x3 pin model

- Implement MPO models in 3x3 pin model

- Develop and implement two-phase models ${ }^{18}$

- Compare AC to BL tools

- Compare coupled tools to data 19

\footnotetext{
${ }^{17}$ Underlying hypothesis is that uncertainty target (for AO less than 3\%) can be achieved by BL code coupling, mesh refinement, and MPO-model improvement for BOA. There is no technical basis) that these items would produce improved solutions.

${ }^{18}$ Comparison to data is vaguely defined (without specification of data and success criteria).
} 
- Perform final UQ for coupled tools

- Implement AC tools for risk assessment

Besides the time constraint (having a decisive demonstration of advancement toward the target by the year 2015), the program is subject to resource constraints.

Comment \#PT.1: While the CIPS challenge problem global objective is clearly defined and quantitative, it has not been decomposed into a defensible set of sub-targets (surrogates/milestones), whose accomplishment provides certain confidence ("case") that the main target (global objective) is enabled.

Comment \#PT.2: Except for "comparisons" (AC to BL, BL to data, AC to data), three entities, namely VERA-BL, VERA-AC, and data are treated in the present workflow as independent components.

Comment \#PT.3: Lack of an integration framework and defensible decomposition (noted in \#PT.1 above) hampers validation data planning. Without a target-oriented development and assessment plan, the validation activity would remain driven by "bottom-up" efforts, and would be fragmented.

\subsection{Decision Model}

Formulation and refinement of a validation data plan require consideration of all key elements of the program, as discussed in the preceding sections. There is a range of technical decisions on validation data and beyond that the VDP has influence upon, e.g., whether it makes sense to invest in acquisition of an identified or new VUQ-grade data set; to what extent a proposed additional experiment would help moving VERA toward the challenge problem's objective or, given severe constraints in experimentation and diagnostics, would it make more sense to change certain models' fidelity and resolution?

With the ultimate goal of basing the VDP decision-making on insights from quantitative sensitivity/uncertainty analysis (SUA), this can only be achieved when the problem's decomposition and integration framework is

\footnotetext{
${ }^{19}$ The four items listed do not constitute a set of surrogates whose completion enables the challenge problem objective (the $3 \%$ AO prediction error target).
} 
defined to certain degree of specificity. Furthermore, given the complexity of the CIPS problem, the SUA machinery is not expected to be functional across the CIPS's multi-scale, multi-physics nature anytime soon. Thus, the CIPS VDP decision-making is expected to remain largely qualitative. Yet, it should be structured, systematic, and scrutable (documented, clear, comprehensive).

Conceptually, the structure of validation has been viewed in term of hierarchy that reflects multi-scale nature of the CASL challenge problems; see Figure 2.9. According to this scheme ${ }^{20}$, value of particular dataset would be determined by its support for the pyramid. However, practical implementation in CIPS has proven a challenge, with a number of issues emerged due to the problem's complexity.

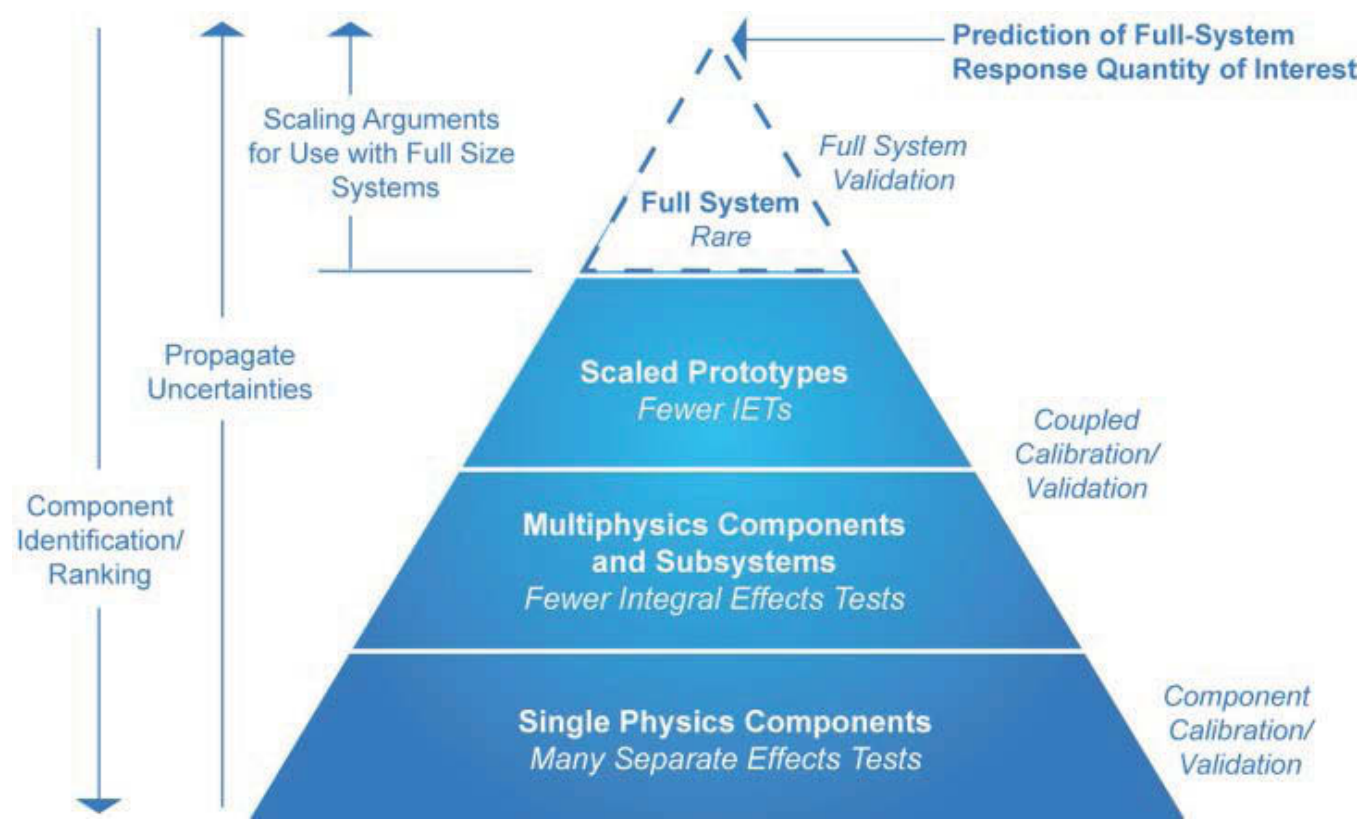

Figure 2.9. A conceptual validation pyramid (CASL proposal, 2010).

Comment \#DM.1: While hierarchical structures are used to describe and characterize a system with increasing complexity, the validation pyramid (as

20 "This hierarchy can be constructed in both a bottom-up and top-down fashion.

Top-down sensitivity analyses can identify the most important systems and components that need to be validated so that the uncertainties at the full-system level are minimized. Cost, schedule, and resources such as experimental facilities can also influence the design of the validation hierarchy." (CASL, 2010) 
shown in Figure 2.9) "mixes" different dimensions of complexity in typical nuclear reactor engineering problems, namely interactions between physics, and interactions between (topological) components. Implicitly it also mixes in the scales. This has caused confusion in identifying and placing phenomena, components, scales, and physics to appropriate levels of the pyramid.

Comment \#DM.2: The validation pyramid creates an impression of AMS capability as a monolithic entity that is subject to testing. This is the case for single-physics e.g., CFD for single-phase flow. However, for problems like CIPS, there are a large number of models involved in each scale and component.

Comment \#DM.3: The validation pyramid treats VUQ of AMS capability similar to testing of a mechanical system, which can be topologically decomposed into weekly coupled subsystems and components. In such "topological decomposition", "lower-level" tests constitute subsystems and components ${ }^{21}$. In reactor engineering problems like CIPS, each physics test represents a self-contained simulation problem, whose objective is to test a physical model, not a system's component.

\section{Recommendation 2.10: Develop validation hierarchy that reflects specificity of multi-physics, multi-scale, multi-component nature of CIPS problem.}

This Recommendation is addressed in Section 4.1.

\footnotetext{
21 "Calibration/Validation/Prediction process is performed many times, hierarchically, towards the full-system predictive capability. The uncertainty from each prediction at the lower levels is propagated upward to be carried along in the full-system calculation. System response quantities-of-interest at the lower levels must be chosen carefully so that they properly interface to the inputs at the upper levels." (CASL, 2010)
} 


\section{CASL VALIDATION DATA STRATEGY}

\subsection{Validation in Nuclear Engineering Context}

Analysis of CIPS-related capability, knowledge and databases reinforces the notion about a wide gap that exists between the emerging class of advanced modeling and simulation tools and supporting databases. This notion is common to new and unresolved technical issues in nuclear engineering. In this Chapter, we articulate a set of perspectives that help to formulate a more pragmatic approach to validation.

\subsubsection{Validation from the Communication Standpoint}

Validation, validation data, database infrastructure, and increasingly, data mining and data assimilation, have become topics of heated debates about application of advanced modeling and simulation (AMS) capability in making important engineering and regulatory decisions. Given extraordinary advances made over the past decades in computational science, computer science, and notably, in affordability of high-performance computing power for industrial applications, it is only natural to expect a broadening role of AMS. Validation and data are central in establishing and assuring that quality of AMS results (measured in uncertainty) are commensurate with the importance of decisions they support. In fact, already during early days of computer simulation, it was recognized that "a decision made without taking uncertainty into account is barely worth calling a decision" (R. Wilson). During late 1980s, the US Nuclear Regulatory Commission, through its group of leading experts (to the largest extent, specialized in thermo-hydraulics), developed so-called CSAU (Code Scaling, Applicability, and Uncertainty) methodology. The CSAU provides guidance on code assessment process including the use of experimental data. The CSAU was applied to "legacy" codes. In the early 2000s, the US NRC issued Regulator Guide 1.203 "Transient and Accident Analysis Methods", which presents the Evaluation Model Development and Assessment Process (EMDAP). Notably, both CSAU and EMDAP provide a framework, whose steps are high level, leaving significant flexibility for applicants to implement the recommendations. The EMDAP aims at modern AMS codes, although the process has not been practiced for them. For more discussion of CSAU and EMDAP, and further references see (Dinh, 2011).

Within the DOE's domain, AMS has been pioneered in defense programs, like Accelerated Strategic Computing Initiatives, or ASCI. The trend has also 
been promulgated to civilian programs. In response to the use of AMS, the US Government Accountability Office (GAO) released a report titled "Nuclear Waste: DOE Needs a Comprehensive Strategy and Guidance on Computer Models that Support Environmental Cleanup Decisions" (GAO-11-143, 2011), which reiterates on the pressing need of "quantifying and communicating uncertainty" when computer model's results are used to support technical and regulatory decisions.

In the decision context, the "communication" aspect of uncertainty is delineated here on equal footing of "quantification" of uncertainty. Applying this notion to validation, the validation activity aims as much at the AMS developers and users (analysts) as at the reviewers, decision-makers, and regulators. It is thus most critical to portray the validation process in a comprehensive, consistent, and complete fashion. Within a graded approach, one would expect that during an early formative stage of the capability, assessments (testing, benchmarks) are designed to help the developers in selecting models, screening significant modeling assumptions, and dealing primarily with model-form uncertainties. At a later stage, however, validation is part of the arguments (the "case") for using certain capability for the application at hand. It has been observed in practice that frustrations and tensions arise when presentation of apparently substantial validation packages fails to convince constructive opponents of the "case". For complex decision and complex phenomena, the "case" is not a collection of tests (code-to-code and code-to-experiments benchmarks). To be convincing, the "case" should communicate a clear objective, validation metric, structured and scrutable approach to achieving the metric, and a body of evidences that this approach is implemented and every element of it is evaluated carefully, with the objective in mind.

Experimental data, plant observations, and other expertise (including results of other codes) constitute the right-hand-side of the above-mentioned body of evidences; the left-hand-side is the AMS.

\subsubsection{Validation - from the Application Standpoint}

Figure 2.4 shows interactions between Application Domain (CP-AD, where reactor calculations are performed and assessed), and Validation Domain (CP-VD), whose mission is to enable VUQ of the application.

First, it is noted that the term "validation" used in the present work denotes activity of assessing maturity of the AMS capability for a specific application, or a well-defined class of applications. It is important to keep in mind that an AMS capability, at best, is validated for an application domain. Using a "validated code" with a poorly defined application domain almost ensures its misuse. 
For engineered and physical systems, AMS-based (design, safety) decision characteristically involve using information about predicted system behavior, such as temperatures, stresses, and degrees of damage, and comparing these "quantities of interest" (QOI) to a design constraint, regulatory limit, or a system capacity, determined from experiments, knowledge base or otherwise computed. This manifests the margin-based decision context (see Figure 3.1).

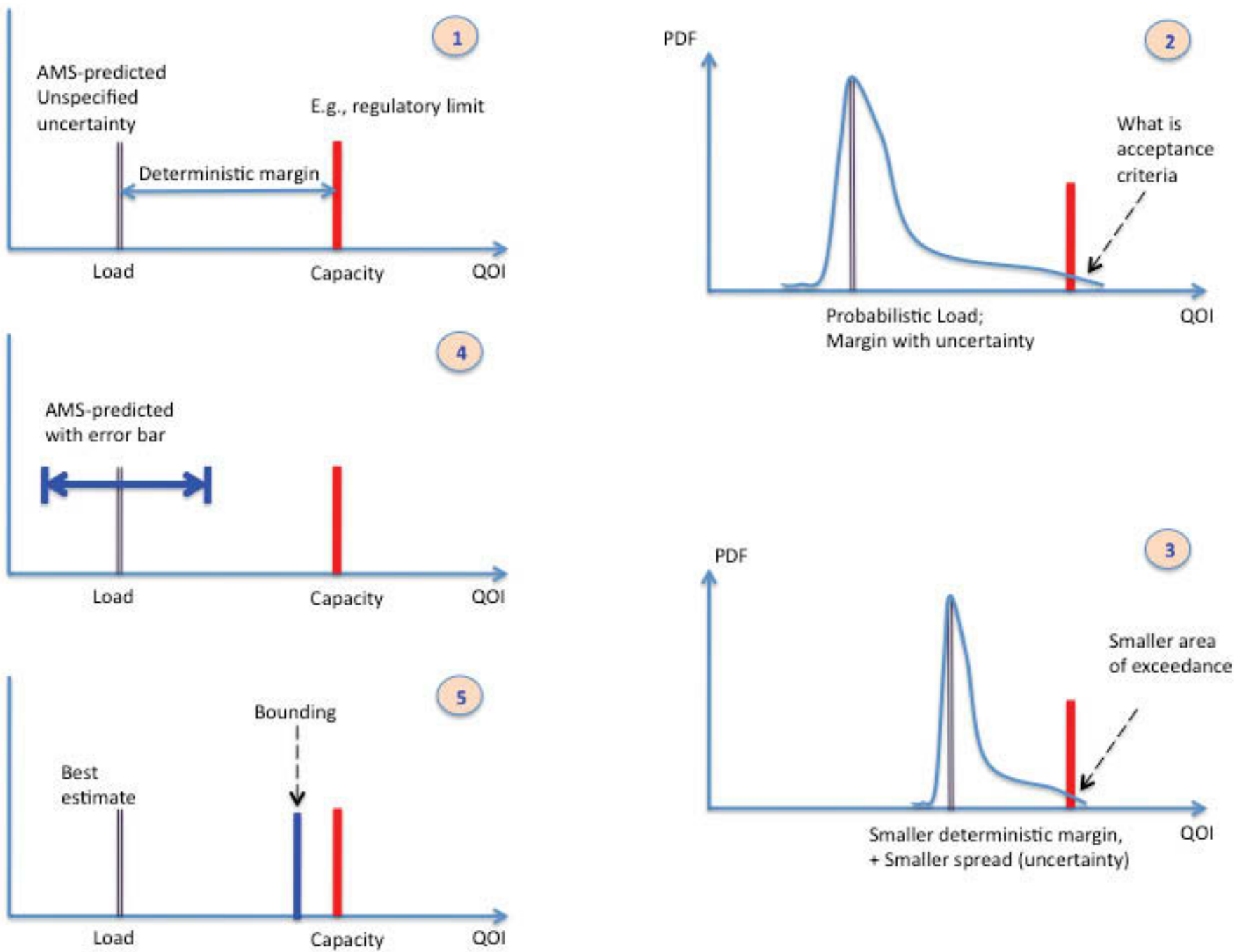

Figure 3.1. Quantitative assessment of calculated results (e.g., safety margin).

(1) - deterministic margin (without UQ).

(2) and (3) - probabilistic loadings. Are they acceptable? Comparing (2) and

(3) for the combined use of margin and uncertainty in engineering decision:

which one is better? The question and answer are meaningful in a riskinformed context that accounts for the risk (including consequence) of exceedance (failure).

(4) - loading prediction with an error bar, without characterization of (non-

zero) probability of failure (i.e., exceeding the capacity line).

(5) - bounding loading that accounts for known sources of uncertainty. 
While a decision analysis discussion would go well beyond the topic of this report, it is worth noting, as will be shown later in CIPS example, that the format by which AMS results are presented and used matters: It imposes different requirements on quantification and communication of uncertainty. For instance, in certain critical applications, it is prudent to base decisions on the bounding value of QOI, e.g. maximum loading that may result under any combination of model parameters over their physically meaningful range.

Within the decision support context, the objective is to enable "quantitative assessment of AMS calculations". Simply, this means the ability to put error bars or distributions, i.e., pdf (probability density function), on the AMS-predicted QOI or, in other words, the UQ.

On one hand, the AMS is subject to uncertainties of different origins and nature (aleatory and epistemic), including

A. Uncertainty in the code's input, including uncertainty in

(A.1) model parameters,

(A.2) scenarios/boundary/initial conditions

B. Mmodel-form uncertainty/biases

C. Numerical treatment uncertainty/error

On the other hand, the resulting uncertainty (distribution) in the predicted value reflects interactions of uncertainties (A-B-C) in, and their propagation through, a complex AMS system. This is to be taken care of by the coupled AMS-VUQ capability in CP-AD. Generally, the AMS capability is expected to evolve to more demanding levels, namely:

○ [L1]: capability to obtain deterministic QOI; Figure 3.1 (insert 1)

○ [L2]: capability to produce "best estimates" calculations (BEC); e.g., using model parameters that reflect the state of knowledge;

○ [L3]: capability to produce "best estimates plus uncertainty" (BEPU) calculations, Figure 3.1. (inserts 2 and 4); and

○ [L4]: capability to produce "best estimates plus reduced uncertainty" (BEPRU); Figure 3.1 (insert 5).

\section{Recommendation 3.1: Establish a risk-informed margin framework for CIPS.}

This Recommendation is further addressed in Sections 4.2.1-4.2.2. 


\subsection{Pragmatic Validation Data Planning}

A foundational element of the CASL project strategy, the VDP should be aligned with the overall CASL plan, VERA requirements and development plan, and VERA validation plan. Conversely, an inefficient and misaligned implementation of VDP would be detrimental to the execution of VERA validation plan, VERA development plan, and CASL mission. Over the past one and a half year, interactions and communications between partners and researchers in CASL have helped a closer-to-common understanding of industry's needs, physics of challenge problems, capability and limitations of VERA-BL codes (ANC/VIPRE-W/BOA) and other tools. A converged understanding of VERA-AC has yet to emerge. At the time of this writing, discussion is ongoing on VERA functional requirements (as part of AMAVRD task) and VERA development/assessment schedule.

The analysis documented in Chapter 2 suggests a pressing need for a more pragmatic approach to validation data planning. The issue was a highlight in various discussions in CASL AMA, THM, MPO and VUQ meetings, meetings with US NRC, and most recently at the February 2012 meeting of the CASL Board of Directors. It has been expressed that simulation tools would be as good as the quality of data that the codes and models are based on, both for calibration and validation.

In the face of the strategic challenge, the following points are considered necessary cornerstones for a constructive discussion of VDP.

(a) For CASL near- and medium terms, the validation data task is directed to support pragmatic decision-making in developmental assessment of CASL products during the VERA-AC's formative phase (... as opposed to a "purist's" pursuit of validation of solidified products)

(b) "Data realism" (... as opposed to "data idealism") is central to the VDP. The provision is that by using advanced data strategies and VUQ tools paramount information value can be extracted from community's knowledge base, including past experiments and legacy codes

(c) The VDP supports a structured, Bayesian inference framework for "total" data assimilation strategy that exploits the "the whole is much more the sum of parts" principle to enable multi-physics, multi-scale validation pyramid; (see Figures 3.2-3.3).

(d) Instrumental for VERA performance, data support is an organic part of the VERA capability and most effective when VERA software 
seamlessly integrates validation databases; CASL Data Center (CDC) is instrumental in implementing this position (see Figure 3.1).

(e) Validation data support is a community-scale effort across and beyond CASL and DOE-NE Programs. The provision is that future VERA users greatly benefit from a systematic, collective and continually growing knowledge base and databases ${ }^{22}$.

\subsubsection{CASL Data Center}

A platform for moving the CASL Validation Data Strategy forward is CASL Data Center (CDC), which provides VERA developers, researchers and users with an effective support of validation data management and usage (see Figure 3.1). ${ }^{23}$

Built on experience and insights from NE-KAMS, the CDC functions include

(i) Validation data inventory and warehouse;

(ii) VUQ-guided data qualification, and

(iii) Data processing for interface with users' data operation, with VERA codes and VUQ workflow, including data assimilation.

The CDC is to be hosted, controlled and enabled by CASL-VOCC infrastructure. Specification and implementation of $\mathrm{CDC}$ hardware and software are to be led by VOCC in coordination with VUQ and other Focus Areas (via Validation Data Committee and Validation Data Product Integrator VDPI). Secure protection and access control (according to technology control plan [TCP] protocols), partners-agreed policy for CDC sustainable operation must be in place and ensured from the get-go.

\footnotetext{
${ }^{22}$ The concept of a DOE-NE-wide and community-scale knowledge base has been promoted and developed under NEAMS NE-KAMS.

23 Work package on CDC's design, initial implementation and integration, demonstration, and application is being formulated and proposed for PoR-5. The CDC initial implementation necessarily focuses on basic functionality of (i)-(iii) above to demonstrate CDC operation in a high-priority development (e.g., subcooled boiling, crud). The objective is for the CDC to become useful for high-level milestones in PoR-6.
} 
There are important cross-cutting issues (see Section 2.5.7) that CDC helps address and a range of benefits that CDC can bring to improve CASL operation in near term, and long term:

- Providing secure, TCP-compliant archives for CASL data

- Providing web-accessible, searchable, quality-assessed, and userfriendly databases

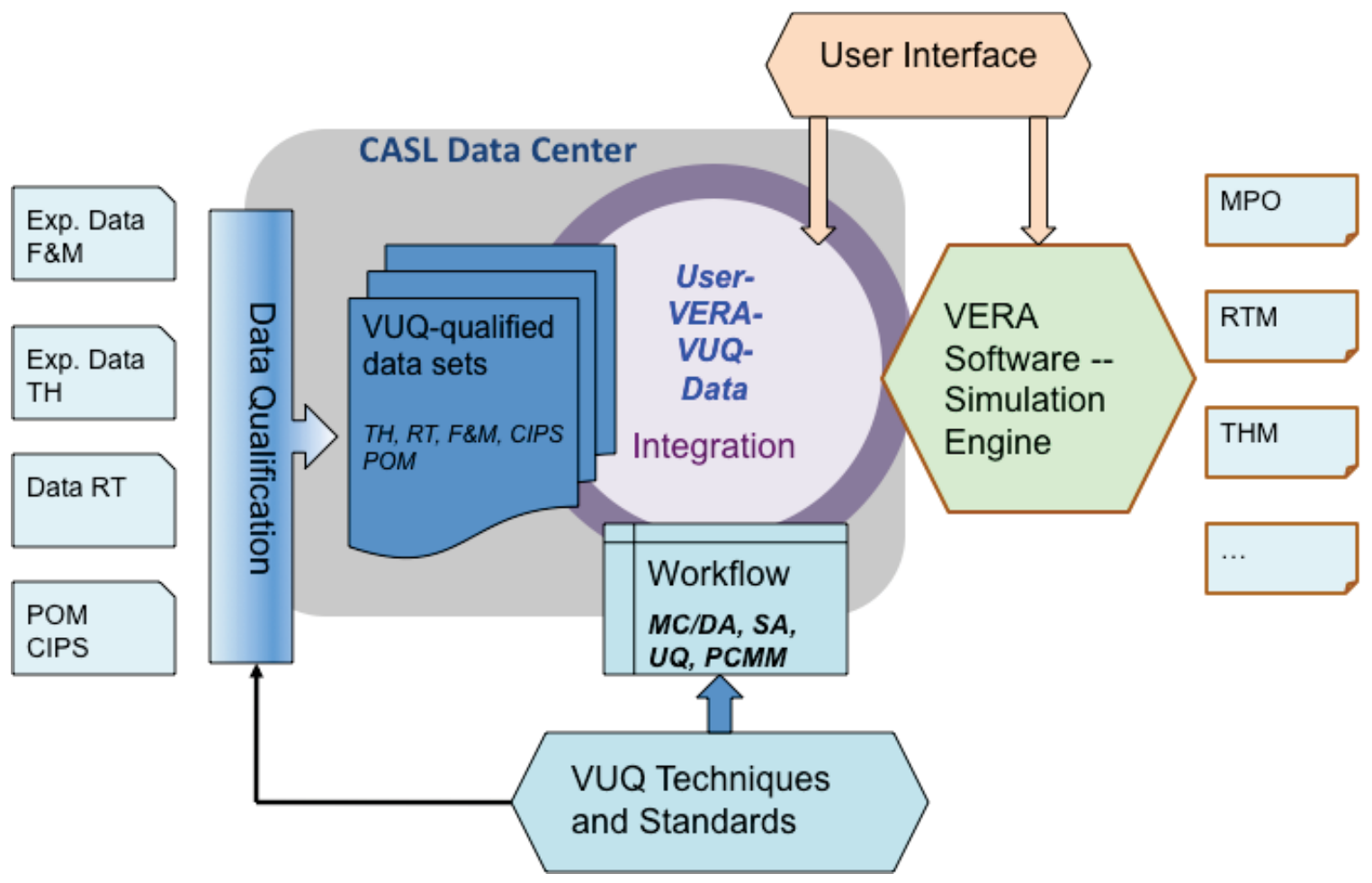

Figure 3.1. CDC components and relations to other CASL functions.

- Making databases compatible to VERA interface and VUQ techniques including data analytics, data mining, and data assimilation

- Providing a sustained platform for making the data (experimental, industrial, computational) collected, qualified and archived during the CASL work available to a broad range of users, including nondevelopers and future users

- Providing the framework for promoting and enabling researchers to adopt and practice standardized and consistent VUQ-guided data processing (data qualification, uncertainty analysis)

- A functional CDC (potentially integrated with NEAMS NE-KAMS) will be a CASL by-product with intrinsic and sustained value for the NE community. 
Recommendation 3.2: Establish and demonstrate CASL Data Center.

Recommendation 3.3: The CDC design and an initial implementation focus on creating data functions, which support a high-priority development in CASL. 


\subsubsection{Application-oriented Total Data Assimilation}

Measuring maturity (quantitative assessment) of the predictive capability for multi-physics/multi-scale applications such as CIPS is the ultimate goal of CASL. This requires integrated use of a wide range of simulation capability and a consistent treatment of uncertainty across disciplines (physics), scales and tools involved in the prediction. This challenge is to be viewed in light of cross-cutting issues (see Comments CI.1-CI.6) discussed in Section 2.5.7.

Two important and distinctive characteristics of CASL data validation strategy are "application-oriented" (Figure 3.2) and "totality" of data assimilation (Figure 3.3). The "application-oriented" (and hence, "fit-forpurpose") is to be contrasted to "general-purpose" validation, which is both demanding (because of unclear parameters domain) and potentially misleading for new applications (as the "validated" code is taken into extrapolative regime).

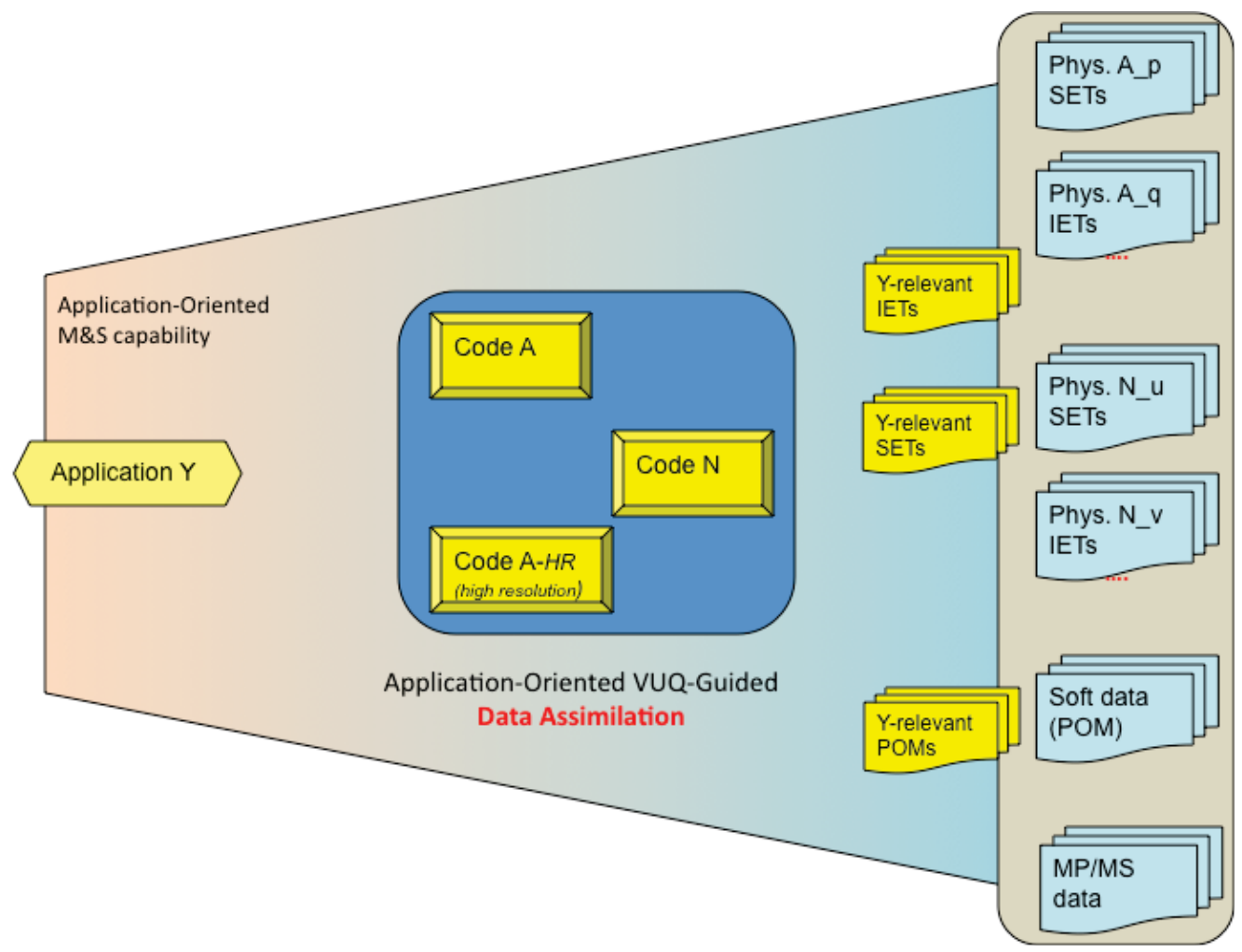

Figure 3.2. Application-oriented model calibration. For Application " $Y$ ", "Y-related" datasets are identified and given higher weight in model calibration. The simulation capability is not "frozen", but is designed to accommodate new data/evidences to the extent appropriate for their Relevance/Scaling/ Uncertainty grades. Determination of "relevance" and weights unavoidably involves expert opinion (subjective). However, the process can be made transparent, traceable/documented, adjustable, systematic, and quantitative. 


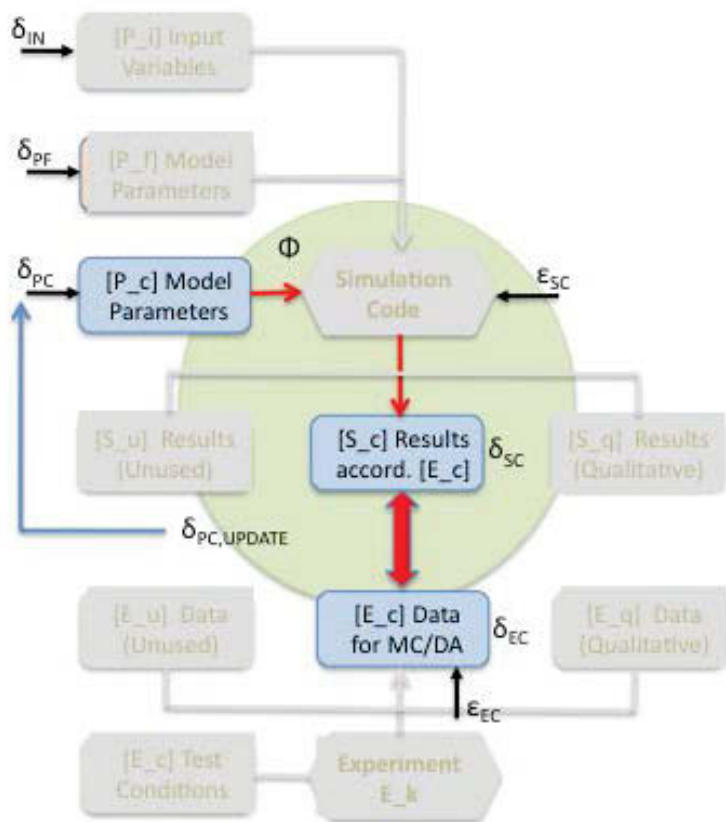

(a) Bayesian calibration (denoted by green circle) on single experiment (compare to Fig.2.4)

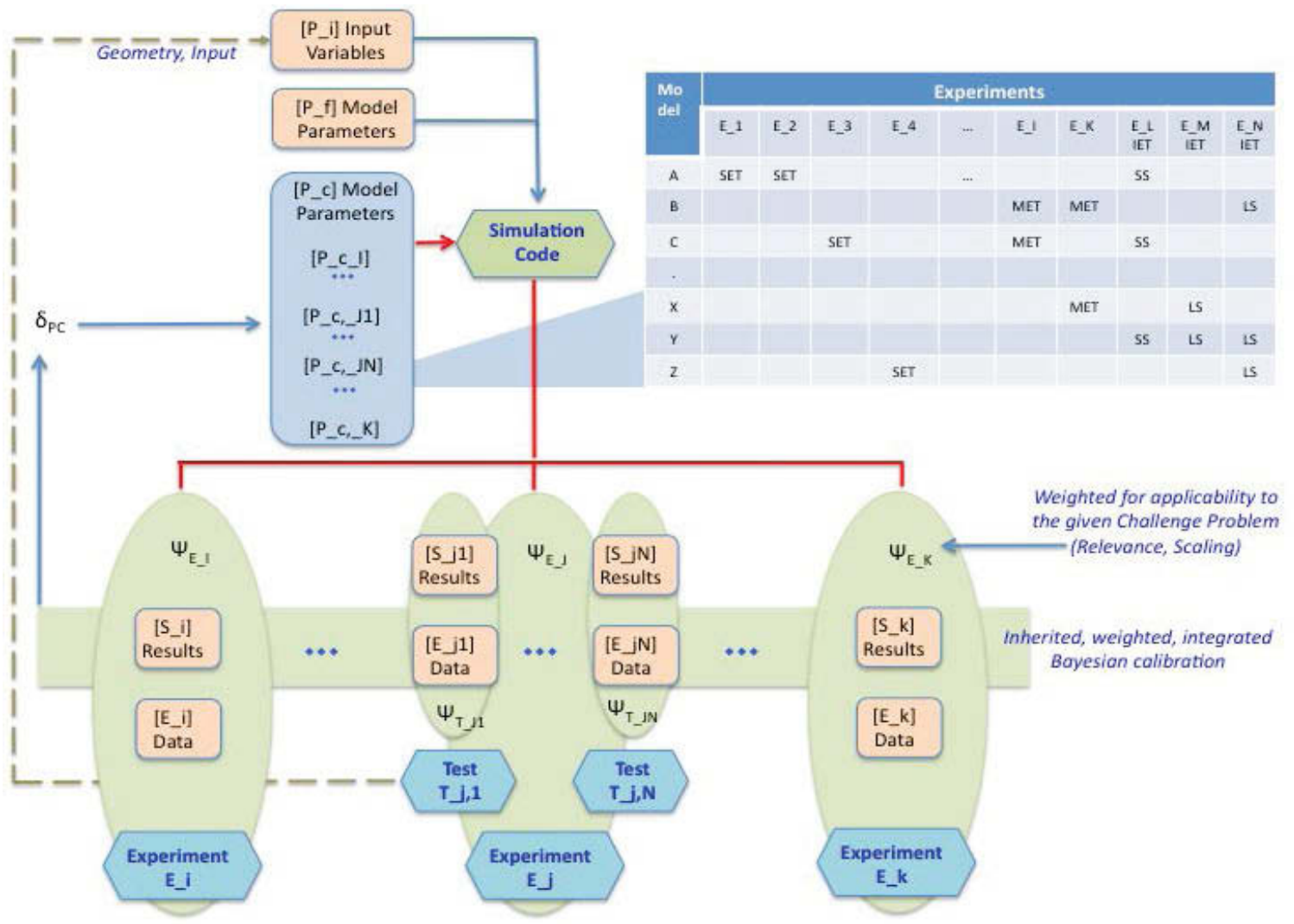

Figure 3.3. (c). "Total" data assimilation, integrating experiments of different origins, $R / S / U$ grades, and individual set of model parameters for calibration.

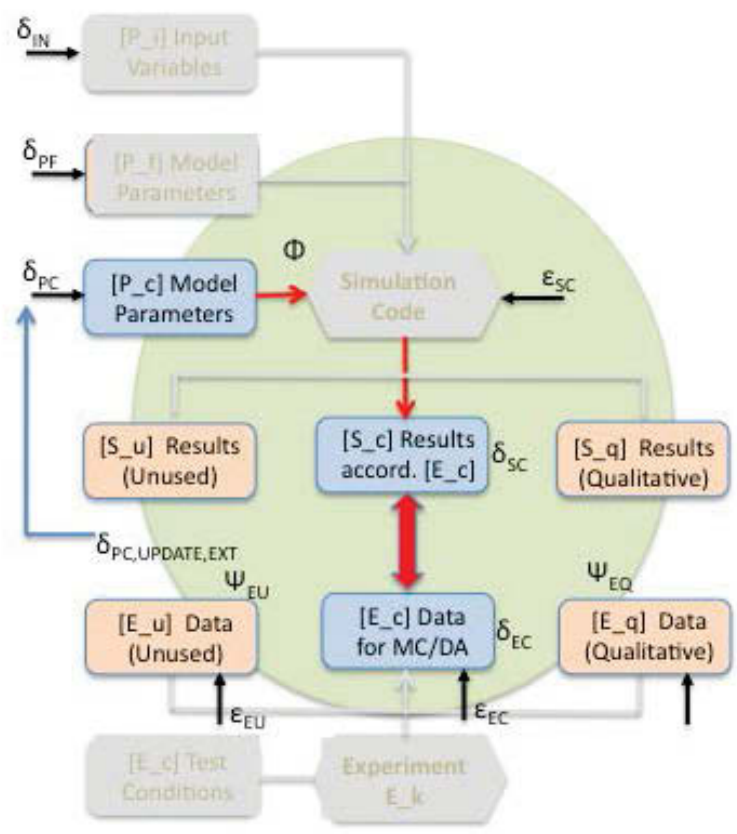

(b) Bayesian calibration that accounts for information from enlarged content of measured and computed data.$$
\text { (1) }
$$ 
A Bayesian inference framework is naturally fit for the task. The process is most capable of accommodating heterogeneous and sparse data sets, providing a means for convenient updating of model parameters with emergence of new data. The framework is also amenable and flexible to changes in model form (e.g., after pushing calibration to limits or having new insights and/or hypothesis). It recognizes that with rapid changes in technology and risk-informed applications (R\&D, design, analysis, licensing), a code validation is not a one-time act (upon a "frozen" code), but a continuing and adaptive process as applications vary and requirements change. More importantly, uncertainties in both simulation and experiments can be accounted for in the Bayesian calibration.

Figure 3.3 (a) highlights modifications from the traditional "plot-to-plot" "viewgraph norm" validation process (typically, ad hoc adjusting parameters) into Bayesian inference process that allows for calibration of a full set [P_c] that accounts for uncertainties in experiments, simulations, and input. Furthermore, the Bayesian process allows incorporation and processing of large data content, including (part of) qualitative insights and normally unused data from experimental measurements and computational simulations. The green circle in Figure 3.3 (b) shows Bayesian calibration for a full set [P_c], accounting for uncertainties in experiments, simulations, and input, and using an extended set of measured data and observations including [E_c] and partial [E_u] [E_q] (weighted with $\Psi_{\mathrm{EU}}$ and $\Psi_{\mathrm{EQ}}$ ). As a result, uncertainty is reduced,

$$
\delta_{\text {PC,UPDATE,EXT }}<\delta_{\text {PC,UPDATE, }}<\delta_{\text {PC, }}
$$

This becomes possible by both advanced Bayesian techniques and affordable computing power, enabling extraction of additional value from past (including legacy) experiments as well as new experiments. Modern SETs use advanced diagnostics (e.g., time/space high-resolution imaging) to produce a wealth of information about dynamic patterns. Another example is integraleffect tests on an advanced plant experiment facility that are designed to record, in each test, over 700 measurement channels over transient processes. The plot-to-plot comparison characteristically utilizes few percents of measured data.

Figure 3.3 (c) shows that the process is further extended to include heterogeneous data and ("messy") evidences, including experiments of different origins, types, and scales (SETs, METs, IETs), plant measurements and observations (PMOs). Processing and characterization of data (evidences) must be performed with care, to assign weights to the evidences and allow them be integrated in the calibration process. Note that the Bayesian framework allows the tests be used within their scope for calibration of corresponding subsets of model parameters (denoted in the figure as 
$\left.\left[\mathrm{P} \_\mathrm{c} \_\mathrm{J} 1\right], \ldots\left[\mathrm{P} \_\mathrm{c} \_\mathrm{JN}\right], \ldots\left[\mathrm{P} \_\mathrm{c} \_\mathrm{K}\right]\right)$ with possible overlaps and consistent updating.

It is instructive to note that as a learning system, implementation of Bayesian model calibration for such a complex, multi-faceted problem (as CIPS) is expected to be substantial, multi-contributor and necessarily gradual in time. A standardized, documented, and inheritable process is critical. A systematic and inherently flexible treatment will bear lasting and communityscale fruits in time.

Calibration must be carried out with the reactor application in mind, using a reactor prototypicality to prioritize evidences.

In Figure 3.4:

[P_SE] - Parameters of models of sub-grid-scale SSG (constitutive relations) for averaged transport model. This parameters set is calibrated on separateeffect tests (SET) and multiple-effect tests (MET). In derivation of SSG models and in design and operation of SET experiments, assumptions are made or idealized conditions are maintained (e.g., about local /quasi-steadystate QSS character of SSG physics, or IC, BC). Consequently, parameters calibrated only on SETs and METs do not fully reflect the effect of system dynamics that presents in integral test facilities and plants. When applied to system simulation, [P_SE] contain two level of errors (local, and integral/dynamical) that need to be corrected $(\theta[\mathrm{SE}])$ through calibration against relevant SETs, METs, and IETs.

$$
\left[\mathrm{P} \_\mathrm{SE}\right]=\theta[\mathrm{SE}]+\varepsilon_{\mathrm{P} \_\mathrm{SE}}+\varepsilon_{\mathrm{P} \_\mathrm{IE}}
$$

[P_IE] - includes all model parameters required in simulation of integral systems (including plants). It contains [P_SE]. The [P_IE] parameters set is calibrated on both SET/MET and small-scale integral-effect tests (SS-IET) and large-scale integral-effect tests (LS-IET). The structural equation modeling method is a potential framework for cross-calibrating SETs and IETs; (Mahadevan and Rebba, 2005; Jiang and Mahadevan, 2009ab; Jiang et al., 2010).

Recommendation 3.4: Based on knowledge base of CIPS-related phenomena, codes, experiments, and plant data, develop a CIPSoriented Bayesian calibration framework, test and demonstrate its performance on surrogate models of multi-physics multi-scale simulation capability. 
Central to the implementation of such framework is consistent integrated treatment of uncertainty across physics and scales, to enable a goal-oriented optimal use of evidences (the goal being "best estimate plus reduced uncertainty" or BEPRU). This requires bringing together different practices in experimentation and uncertainty analysis; even the same term used varies in significance for different disciplines. For instance, $2 \%$ errors of model parameters would be large in one area (e.g., core neutronics), whereas $20 \%$ error is typical in another (e.g., boiling heat transfer). Note the applicationoriented processing of evidences that requires grading and weighting of same data sets needs to be revisited for a new application when it changes the prototypic baseline used for scaling (see Figure 3.4 caption).

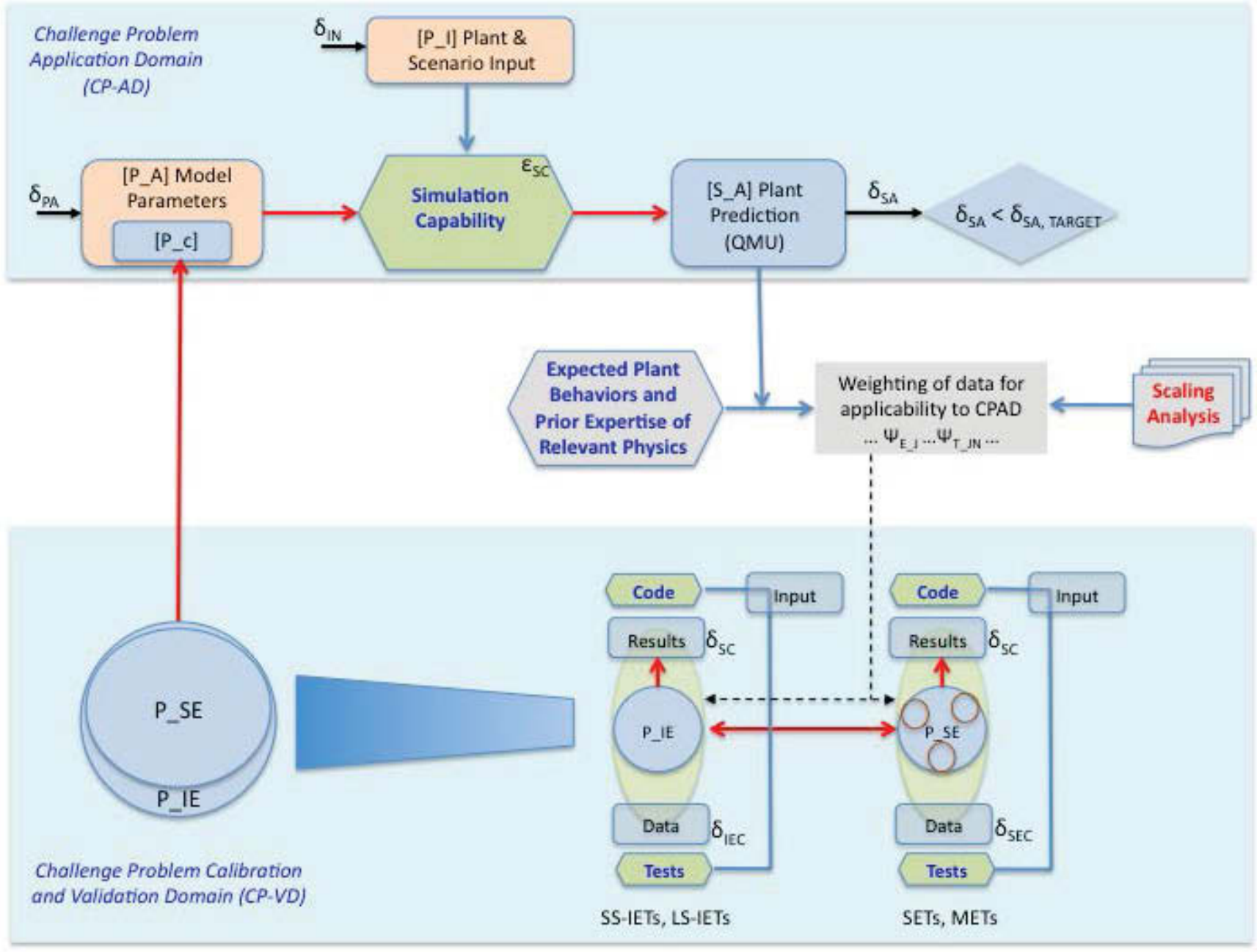

Figure 3.4. "Total" data assimilation as an iterative process involving fullscale simulations. Results calculated for plant scenarios and scaling analysis are used to determine relevance and weight contributions of different tests and datasets. This emphasis on scaling reflects specificity of nuclear reactor engineering (particularly safety-related) applications where calculations are 
necessarily made in extrapolative regime beyond domain of data available for model calibration. Scaling too is inherently limited by experts' knowledge base e.g., with potential for ignoring physical mechanisms and effects, which may become dominant under reactor prototypic conditions. Since knowledge base is largely built on analogy, SETs and IETs, which are subject to simplifications and potentially misleading.

\subsubsection{Qualification and Integration of Heterogeneous Data}

To reduce uncertainty in model forms and model parameters, e.g., narrowing their distributions (pdf or applicability intervals), models and simulation codes are benchmarked and calibrated against applicable experiments. Through the Bayesian framework, a body of experimental data becomes assimilated in the AMS capability. Critical for the success of the "total data assimilation" approach are identification, processing, qualification, and appropriate integration of a necessarily substantial and large body of data and evidences of all kinds, including "messy", poorly conditioned and heterogeneous data ${ }^{24}$. The keyword here is "appropriate integration". Data "realism" emphasizes that not all experiments are "born equal", i.e. equally useful for the application at hand.

Generally, effectiveness of data assimilation depends on

a) Availability (quantity, reproducibility) of applicable experiments

b) Degree of applicability of experiments (material scaling, geometric similarity, physics scaling)

c) Quality of experimentation: characterization of uncertainty in experimental (initial, boundary) conditions

d) Scope of measurement: diversity of diagnostics, number of measuring channels, temporal and spatial resolutions

e) Quality of measurement: characterization of uncertainty of measured data.

Subject to the above characteristics, data sets obtained in experimental programs vary greatly by their format and VUQ quality. To determine relevance and significance of certain tests for assessing maturity of an AMS

24 "Messy" (poorly conditioned, unstructured) data e.g., plant observations and industry's insights are important qualitative evidences to be integrated in the validation case, including their potential use for "invalidating" models. Validation failures are valuable (more than "another confirmation") in discerning limitations of modeling assumptions and determining the model applicability domain. 
tool for a given application, subject matter experts are required. Even then, careful examination of the application, the models and experiments involved are needed for subject matter experts to provide credible judgment. This expert evaluation process constitutes a major inherent source of (epistemic) uncertainty.

Furthermore, in order to evaluate the impact of the uncertainty on predicted QOIs, it requires that the epistemic uncertainty be quantitatively determined, integrated and propagated toward QOIs. The Relevance/Scaling/Uncertainty R/S/U grading approach proposed in Table 3.1 (Dinh, 2011) is a step in this direction. However, there are other gaps to bridge. The $R / S / U$ grade system should be made related to uncertainty representation, so that the grade (expert opinion) can be numerically integrated and quantitatively propagated in UQ.

Table 3.1. Grading of experimental or plant test data by their VUQ quality.

\begin{tabular}{|l|l|l|l|l|}
\hline \multirow{2}{*}{$\begin{array}{l}\text { QUQ } \\
\text { Quality }\end{array}$} & \multicolumn{1}{|c|}{ Grade } \\
\cline { 2 - 5 } $\begin{array}{l}\text { Relevance } \\
{[\mathbf{R}]}\end{array}$ & $\begin{array}{l}\text { Very High } \\
\text { (direct) }\end{array}$ & High & Medium & Low \\
\hline $\begin{array}{l}\text { Scaling } \\
\text { [S] }\end{array}$ & $\begin{array}{l}\text { Prototypic } \\
\text { (full-scale) }\end{array}$ & $\begin{array}{l}\text { Adequately } \\
\text { scaled }\end{array}$ & Medium & $\begin{array}{l}\text { Inadequately } \\
\text { scaled (large } \\
\text { distortions) }\end{array}$ \\
\hline $\begin{array}{l}\text { Uncertainty } \\
\text { [U] }\end{array}$ & $\begin{array}{l}\text { Well- } \\
\text { Characterized }\end{array}$ & Characterized & Medium & $\begin{array}{l}\text { Poorly- } \\
\text { Characterized }\end{array}$ \\
\hline
\end{tabular}

To enable the integrated uncertainty analysis, two key measures are used: RPP, a Reactor Prototypicality Parameter, and EMU, an Experimental Measurement Uncertainty. In an analogy, one may align the RPP with "accuracy" (global applicability) and the EMU with "precision" (local variability).

RPP measures how close a given test conditions are to the reactor conditions in scenarios of interest to the application (in CP-AD; Figure 3.4). By appropriate choice of RPP, the "closeness" ensures both relevance and scaling.

We propose to define prototypicality as ratio of the governing scaling parameter $\mathrm{Sc}_{\text {Mod_K }}$ for "model of physical process $\mathrm{K}$ " calculated for 
test/experimental conditions, [S $\left.\mathrm{Sc}_{\mathrm{Mod}_{-} \mathrm{K}}\right]$ EXP and reactor application's conditions $\left[\mathrm{Sc}_{\text {Mod_K }}\right]_{\text {APP. }}$.

$$
\mathrm{RPP}=\left[\mathrm{Sc}_{\text {Mod_K }}\right]_{\mathrm{EXP}} /\left[\mathrm{Sc}_{\mathrm{Mod} \_} \mathrm{K}\right] \mathrm{APP}
$$

Typically, the scaling for physical process $\mathrm{Sc}_{\mathrm{Mod}} \mathrm{K}$ is a dimensionless group (or groups) e.g., $\mathrm{Re}, \mathrm{Gr}, \mathrm{P} / \mathrm{P}_{\mathrm{CR}}$, or ratio of time scales of competing subprocesses. This scaling parameter characterizes conditions that (are known or judged to) govern the physical process described by the model under testing.

Evidences at high RPP (e.g., plant data, RPP=1; LS-IET), even under characteristically large in-reactor measurement and "process condition" uncertainty, are taken with high significance in engineering applications, e.g., in "safety case". Experiments with RPP $>0.1$ are taken as large-scale, as having high potential to preserve the governing physics ("of physical process K").

Similarly, for a given test, EMU is an experts' evaluation of the experiment's design, construction, conduct and measurements. Information value of a dataset increases with its quality. As a measure of belief in the experimental quality, EMU may be defined as $10^{1-\mathrm{N}}$, where $\mathrm{N}$ is the uncertainty grade in Table 3.1 .

Thus, EMU $=1 \quad$ - uncertainty is so large that the trend is not discernable.

$\mathrm{EMU}=0.1 \quad$ the trend is discernable but low-quality data.

$\mathrm{EMU}=0.01-$ the trend is clear, good-quality data.

$E M U=0.001-$ high-quality VUQ-grade data.

Note that while RPP and EMU provide a means to harmonize different experiments, different types and quality of evidence, formulation of RPP in each specific case is subject to expert opinion, and associated limitations. However, given a documentation and peer review process, and flexibility of the Bayesian inference method to be updated, the resulting quantitative analysis provides capability to deal with a complex problem like CIPS with its heterogeneous multi-faceted body of data (Figure 3.5).

For the CIPS's initial implementation, the weight factor $\Psi_{\mathrm{E}_{-} \mathrm{J}}$ of dataset "J" (physics "K") can be expressed as

$$
\Psi_{\mathrm{E}_{-} \mathrm{J}} \sim\left(1-\mathrm{EMU}_{\mathrm{J}}\right)^{\mathrm{m}}\left[\mathrm{RPP} \mathrm{K}, \mathrm{J}^{\mathrm{n}}\right.
$$

For $\mathrm{m}=\mathrm{n}=1$, a low-quality data $(\mathrm{EMU}=0.01)$ plant data $(\mathrm{RPP}=1)$ is weighted equally as a K-relevant set of VUQ-grade data "J" obtained in experiment at scale $1 / 100$. This relative weight can be adjusted, e.g., $n=2$ $(m=1)$ to assign higher significance to scaling RPP. Note that there is a range 
of physics for that the plant evidence is relevant, whereas no other tests exist $(\mathrm{RPP}=0)$.

Implementation on the integrated uncertainty treatment as part of a Bayesian calibration framework for CIPS (Recommendation 3.3) will give insights needed to adjust the numerical grading of RPP and EMU, and weighting formulae, so that grading and weighting help measure uncertainty in making the "validation case" for VERA. It is expected that the formulation can be optimized to be more effective in different classes of problems, with their respective decision priority, set of codes and body of data support.

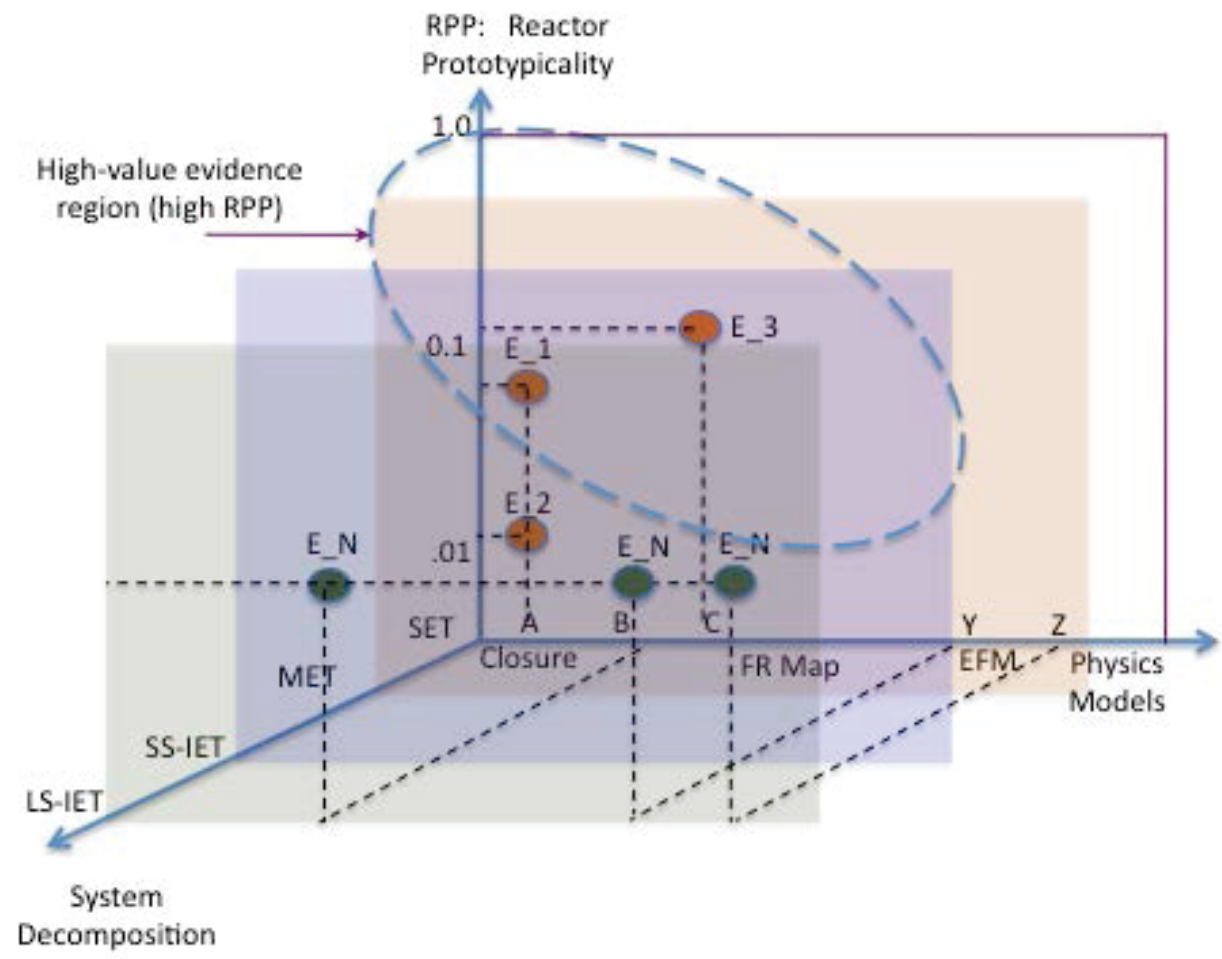

Figure 3.5. Validation Cubic shown for two-phase thermal-hydraulics over a range of scales from sub-grid-scale SGS (Closure) to macroscale (effective-field model EFM) dynamics. Experiments included $\left(E_{-} 1, \ldots\right.$ $\left.E_{-} N\right)$ are notional. See also a table in Figure 3.3(c) for notional characteristics of these experiments. The status of validation data supports is correlated with "filling" of the Cubic's upper layer (i.e., RPP $\rightarrow$ 1) across Physics and System Decomposition dimensions.

Instrumental to the credibility is comprehensiveness, consistency, and completeness of documentation of knowledge base, evidences, heuristic and quantitative arguments (e.g., about relevance and applicability of evidences) 
and experiments used in suggesting the range and the distribution (pdf) of the uncertain parameters. In the Bayesian inference framework, emergence of quality evidences (e.g., VUQ-grade experimental data) and their integration in the "case" should lead to narrowing down the range and distribution of uncertain parameters.

\section{Recommendation 3.5: Investigate options and consequences of grading and weighting systems on model calibration and uncertainty analysis.}

\subsubsection{Code-to-code Cross Fertilization}

The proposed CASL validation data strategy focuses on supporting the VERA-AC development under severe data deficit. An important element of this strategy is to make a more systematic, VUQ-guided use of code-to-code (C2C) comparison that accelerates maturation of VERA-AC tools. It recognizes that $\mathrm{C} 2 \mathrm{C}$ activity is not a replacement for validation in any sense, but it can serve the developmental phase for the new tools. In fact, CASL researchers in technical areas (THM, MPO) have used and plan to exploit the "code-to-code comparison".

Notably, this C2C comparison is most helpful in the face of woefully deficient databases needed for assessing and calibrating models of micro- and meso-scale processes modeled in high-fidelity/high-resolution simulation tools (e.g., Hydra-TH, MAMBA) in VERA-AC. As shown below in Chapter 4 , the number of models and model parameters (in CMFD/SFB, CRUD) are significantly larger than the amount of tests and data available for model evaluation. As a result, benchmarks of VERA-AC against a few macroscopic tests are not fruitful an exercise for discerning limitations of the micro/mesoscopic models. And yet, during the developmental phase, such qualification (primarily, determining domain of applicability of key micro-physical modeling assumptions) is central to model selection. In other words, benchmarks against a small set of macro-scale experiments with integral measurements and poorly-controlled micro-scale (surface) conditions neither help identify model-form uncertainty nor be adequate for model-parameter calibration.

The key idea of VUQ-guided $\mathrm{C} 2 \mathrm{C}$ comparison is to facilitate crossfertilization of VERA-BL and VERA-AC simulation capability that help identify major inconsistencies in VERA-AC models and inform coarse-grain models in VERA-BL. Calibrated to match VERA-BL basic capability e.g., in simulating macro-scale experiments, the VERA-AC tools are capable of providing far more mechanistic details, including spatial distribution. The 
result can be used to examine homogenization/coarse-graining assumptions or closure relations used for VERA-BL.

In turn, the state-of-the-practice and other available codes (VERA-BL and VERA-SC), both individually and as coupled tools, are used to perform simulation of select scenarios and tests. The resulting "numerical experiments" database is sufficiently rich and relational that can challenge VERA-AC models in a way few tests with integral measurements cannot.

Recommendation 3.6: Develop and implement a plan for using VERABL-generated numerical experiments for aiding developmental assessment of select VERA-AC tools, and their multi-physics capability. 


\section{CIPS VALIDATION DATA PLAN: UPDATE AND IMPLEMENTATION}

\subsection{CIPS Phenomenological Hierarchy and Decomposition}

\subsubsection{Multi-physics Multi-scale Hierarchy}

A hierarchical representation of CIPS is necessarily multi-dimensional, reflecting hierarchy within each of the participating physics (TH, CC, RT, and FC) and their inter-physics interactions. Certain multi-physics interaction are weaker, others are stronger (shown as dark magenta), like TH-CC, RT-TH. The inter-physics interactions occur at respective scales, forming own hierarchy.

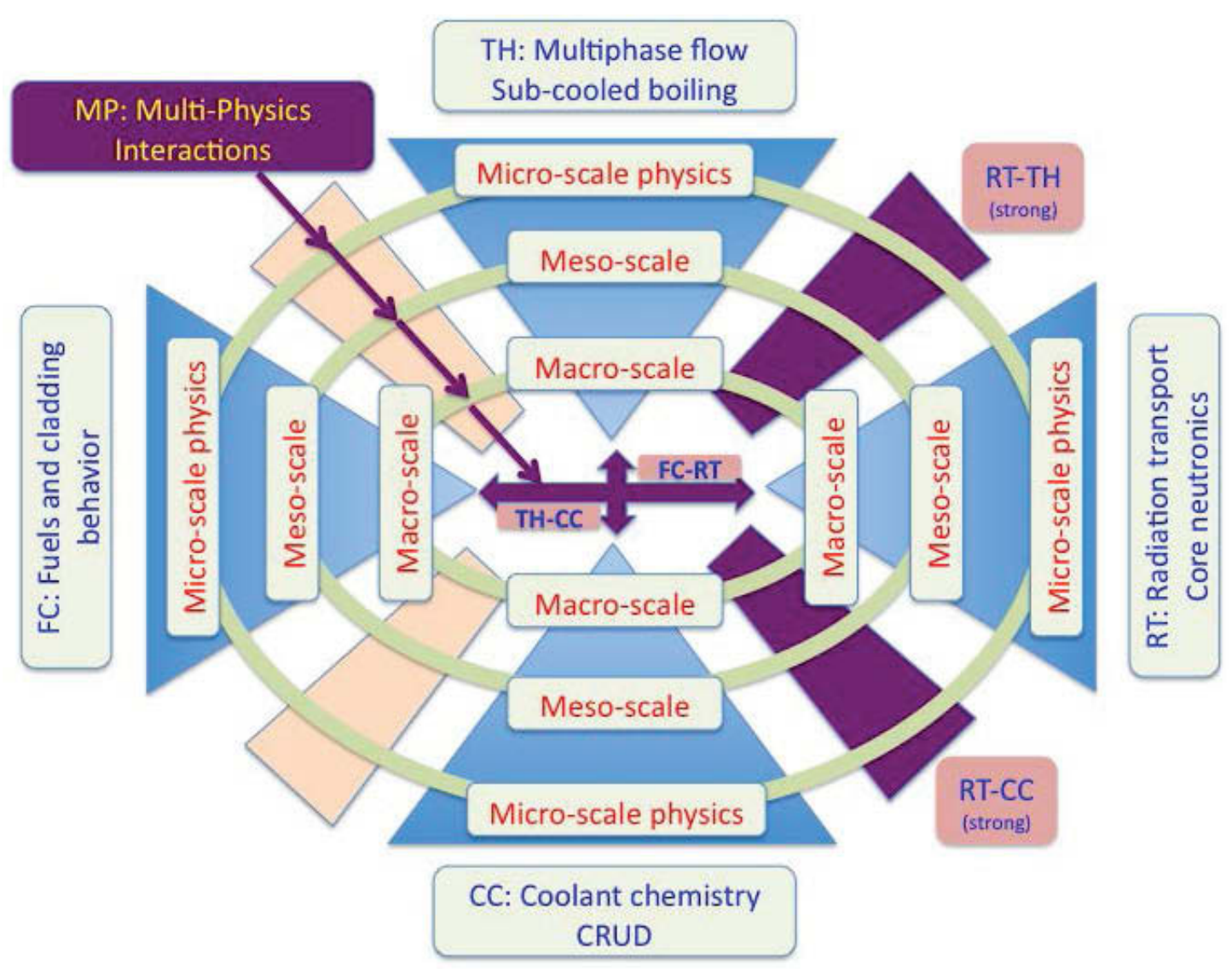

Figure 4.1. Multi-physics, multi-scale decomposition of CIPS. 
Sections 4.1.1, 4.1.2 and 4.1.3 provide hierarchical decomposition for $\mathrm{TH}$ and $\mathrm{CC}$ areas, and $\mathrm{TH}-\mathrm{CC}$ (strong) interaction, respectively. This decomposition is based on a systematic treatment of detailed multi-scale phenomenology involved in TH and CC, with respect to CIPS. Sub-section 4.1.4 briefly discusses other technical areas of the framework shown in Figure 4.1 .

\subsubsection{Thermal-hydraulics (sub-cooled flow boiling)}

Hierarchically, TH AMS capabilities of interest to CIPS are inclusive of

- Atomistic/MD (surface physics, coolant chemistry) of importance to, e.g.,

- Nucleation

- Wettability (triple contact line)

- Micro-hydrodynamics

- Evaporating meniscus

- Deposition

- Thin film (breakup/coalescence)

- Continuum with interface tracking

- Bubble/interface dynamics

- Turbulence

- Turbulence-interfacial surface interactions

- Inter-field exchanges ... closure physics

- Mass (evaporation/condensation)

- Momentum

- Energy

- Two-phase averaged model

- Flow pattern

- Gradient across flow channel

-Multi-dimensional effect

- Domain decomposition coupling between models of different formulation

- Interface of models of different fidelity/resolution

As currently composed, the THM activities are multi-faceted involving a wide range of scales and processes/phenomena whose models need calibration, from turbulence to nucleation to condensation rate in subcooled flow boiling. Time-averaged (effective-field) essentially-1D models (like in RELAP, VIPRE), time-averaged 3D models (in CMFD codes), and LES/ITM codes (which do have delicate modeling in it too)-- each class involves modeling of mechanisms whose characteristic time scales vary so broadly that each code class requires fundamentally different data and hence separate diagnostic techniques. 


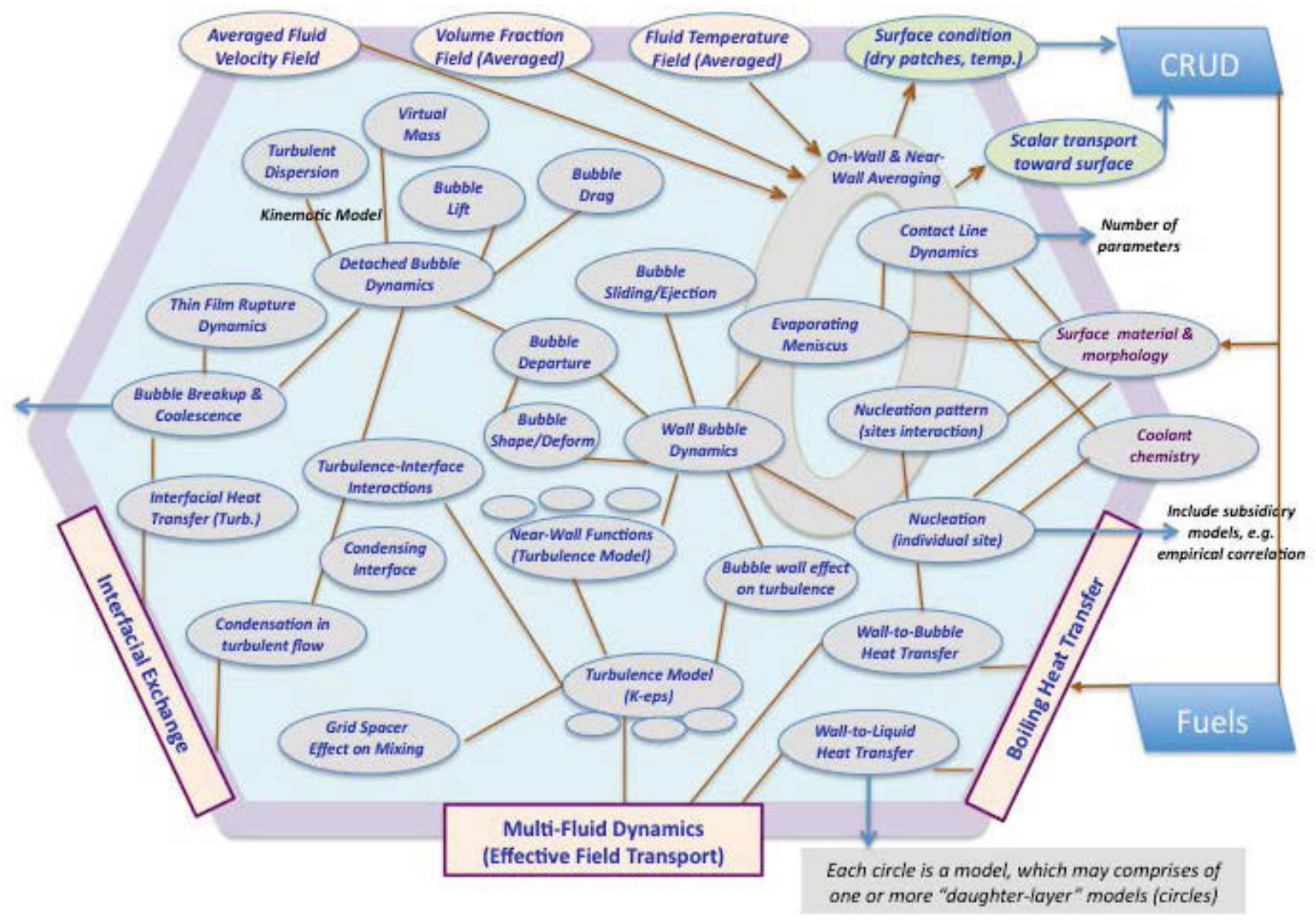

Figure 4.2. Sub-cooled flow boiling phenomenology: decomposition and interactions (not all shown) ${ }^{25}$. Each circle (phenomenon) can be further decomposed, and described by sub-models and model parameters.

It is noted that for engineering problem solution, capability is not assessed for fidelity (physics details) or resolution (spatial details), but for its "fitnessfor-purpose". For CIPS problem, the purpose of TH capability is to predict, with required accuracy and computational efficiency, thermal-hydraulics quantities of interest (QOI) in sub-cooled flow boiling regime (Figure 4.2), namely

Density (void fraction)/temperature field

$\rightarrow$ Providing input to RT

Fluid velocity field,

Scalar transport in near-wall bubbly layer/sub-layer

$\rightarrow$ Providing input to CC-transport, system-level T-H

25 In-depth discussion of multiphase flow and subcooled flow boiling phenomenology and sources of uncertainty is provided in a separate report (VUQ.VVDA.P05.4). 
Cladding surface wetting and temperature

In local dry patches (contact line dynamics)

$\rightarrow$ Providing input to Fuels, CRUD

Clearly, "mechanistic" treatment of the phenomenology shown in Figure 4.2 is necessarily heuristic and ad hoc. Individual models are empirical, based on observations made at non-PWR non-CIPS conditions. Many processes (e.g., nucleation) are not well understood, stochastic, or not quantifiable; Table 4.1. 


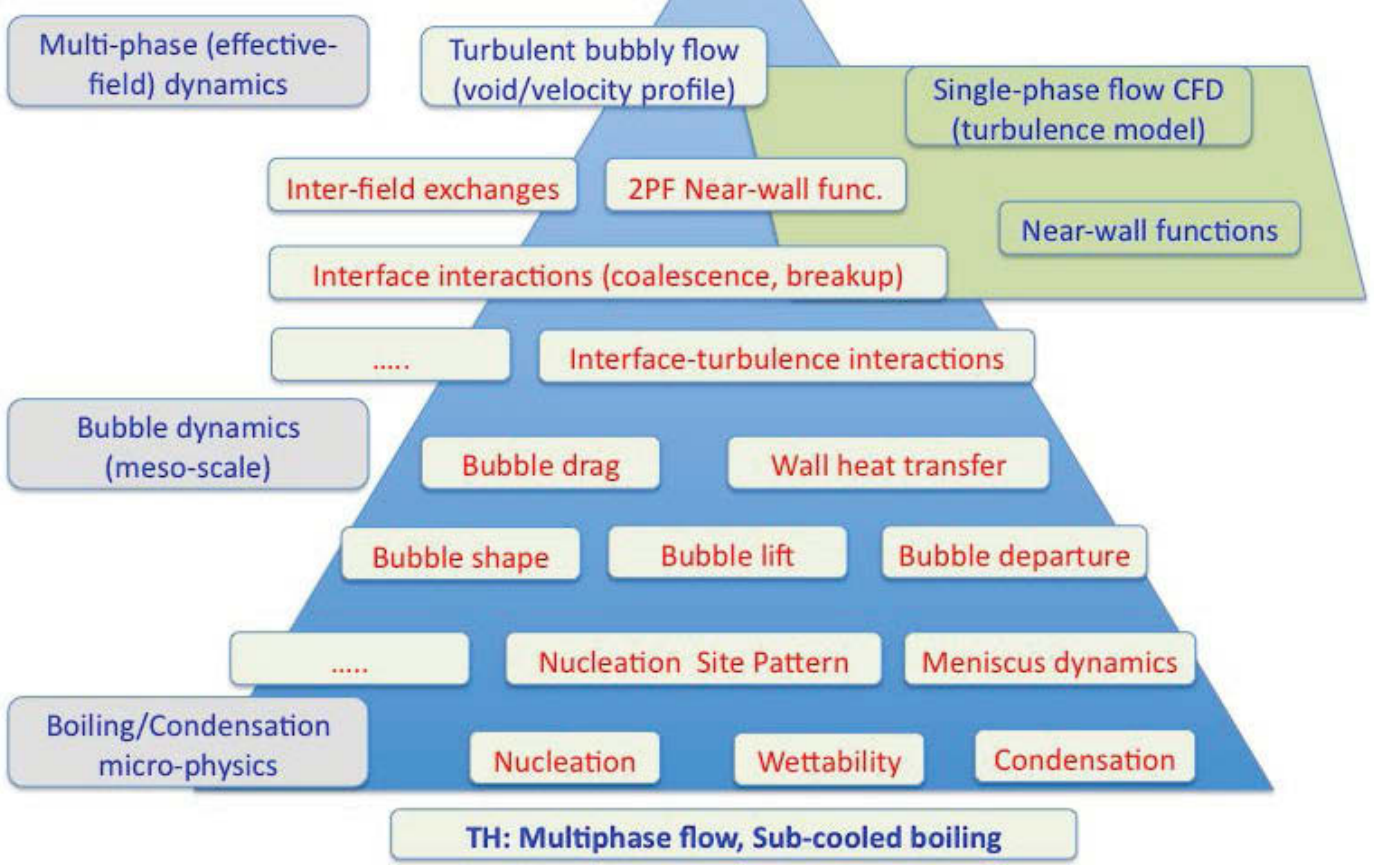

Figure 4.3(a). Thermal-hydraulics dimension of the CIPS framework (Fig. 4.1).

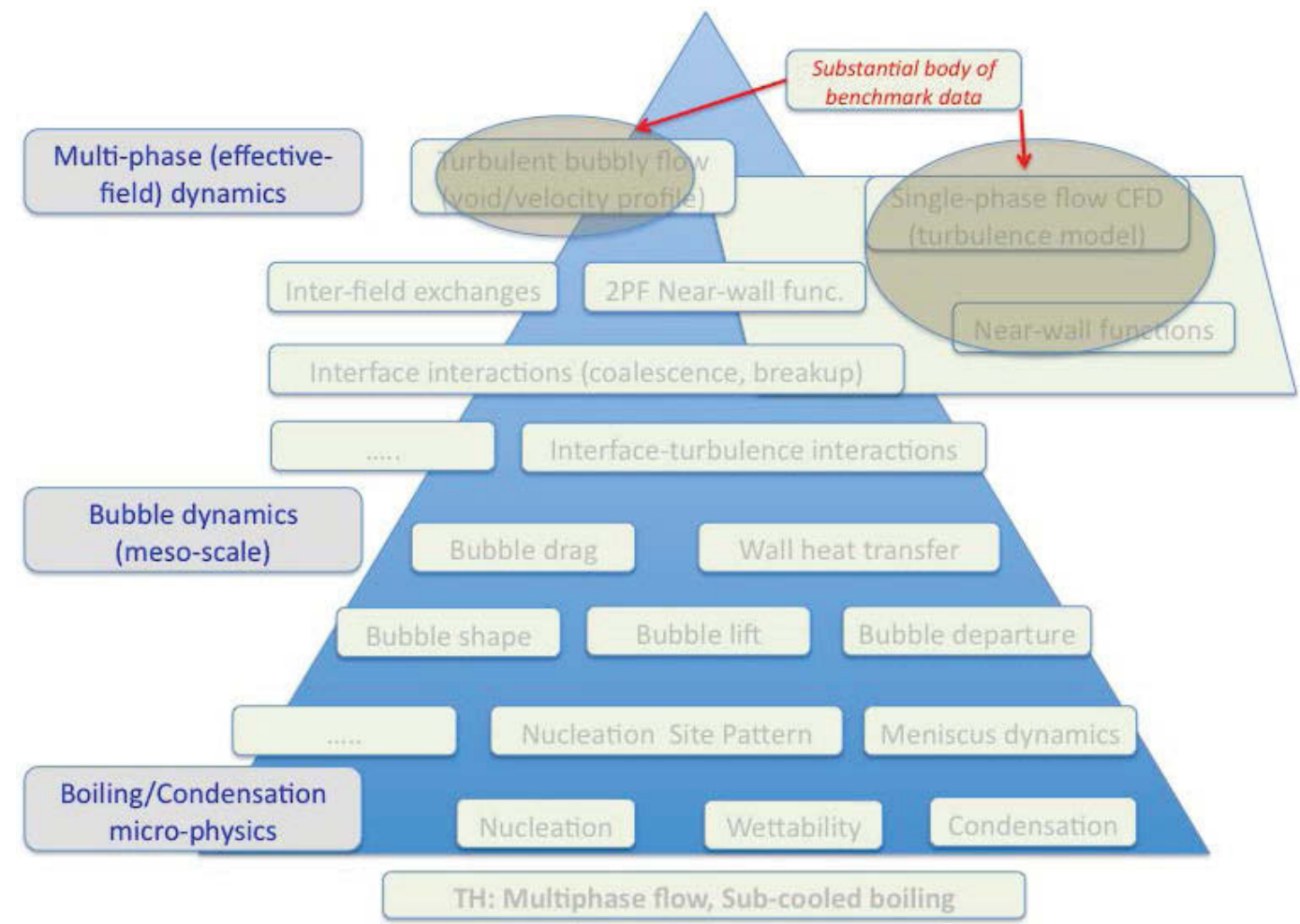

Figure 4.3. (b) Circles show areas addressed by current sets of CFD and CMFD benchmarks (Section 2.5.3). 
Figure 4.3(a) depicts thermal-hydraulics (TH) dimension of the CIPS framework (shown in Figure 4.1). Shown are selected phenomena that appear to present significant uncertainty to the prediction of QOIs. Note that for the CRUD problem, single-phase flow CFD is a necessary part of the picture, but its validation plays only limited role for two-phase flow simulation capability. Turbulence models and near-wall functions that are "validated" for singlephase CFD don't lend themselves automatically to two-phase flow. The redfont boxes are where models are used but not individually assessed/validated.

Figure 4.3(b) shows areas that are relatively well covered by the planned benchmarks (listed in Section 2.5.3). Severe lack of data for qualification and calibration of closure relations, sub-grid-scale models, and wall functions such as turbulence in multiphase systems (with phase change), boiling heat transfer (with surface physico-chemistry effect), condensation (in turbulent flow) under PWR operating conditions.

Table 4.1. References of relevant boiling experiments with data about mesoscale mechanisms involved in SFB. No systematic investigation. With one exception, the experiments were poorly scalable to PWR-CIPS conditions. Tests had limited diagnostics, without resolving near-wall structures and detail dynamics. Only a faction of phenomena (in Figure 4.2) is investigated. Those processes studied exhibit lack of understanding, large uncertainty, and great variability. See Table 3.1 for scaling grades.

\begin{tabular}{|c|c|c|}
\hline Authors (year) & Phenomena investigated & Scaling \\
\hline Bertel et al (2001) & Interfacial area in SFB & 1 (1 atm) \\
\hline Garnier et al (2001) & Local measurements & $1(\mathrm{R} 12)$ \\
\hline Kang et al (2002) & Vapor phase measurements SFB & $1(\mathrm{R} 113)$ \\
\hline Warrier et al (2002) & Interfacial heat transfer & $1($ low P) \\
\hline Roy et al $(1992,1997)$ & Turbulence, void fraction & $1(\mathrm{R} 133)$ \\
\hline Chen et al (2003) & Bubble coalescence & $1(1 \mathrm{~atm})$ \\
\hline Yeoh et al (2004) & Bubble departure, bubbly flow & $1(1-2$ atm $)$ \\
\hline Okawa et al $(2005 a, b)$ & Bubble slide & 1 (1 atm) \\
\hline Maurus et al (2006) & Bubble; boundary layer & 1 (horiz.) \\
\hline Bang et al (2004) & Visual bubble & $1(\mathrm{R} 134 \mathrm{a})$ \\
\hline Situ et al $(2004 a, b)$ & Bubble dynamics & $1(1 \mathrm{~atm})$ \\
\hline Unal (1976) & Bubble growth & $3($ full P) \\
\hline Chang et al (2002) & Wall bubble & $1(\mathrm{R} 134 \mathrm{a})$ \\
\hline Basu et al (2005) & Wall heat partitioning in SFB & $1($ low P) \\
\hline Basu et al (2002) & Boiling onset, nucleation site density & $1($ low P) \\
\hline Hibiki \& Ishii (2003) & Nucleation site & $1(1 \mathrm{am})$ \\
\hline Theofanous et al (2001) & Nucleation on different surfaces & $1(1 \mathrm{~atm}, \mathrm{~PB})$ \\
\hline Dinh et al (2004) & Nucleation & $1(1 \mathrm{~atm}, \mathrm{~PB})$ \\
\hline
\end{tabular}




\subsubsection{Coolant Chemistry and CRUD}

Figure 4.4 depicts a framework for describing CRUD and CRUD-related coolant chemistry, and corrosion products phenomena and their interactions. Shown in this framework are those factors and phenomena (themselves being aggregate) perceived to be dominant in their significance and/or uncertainty.

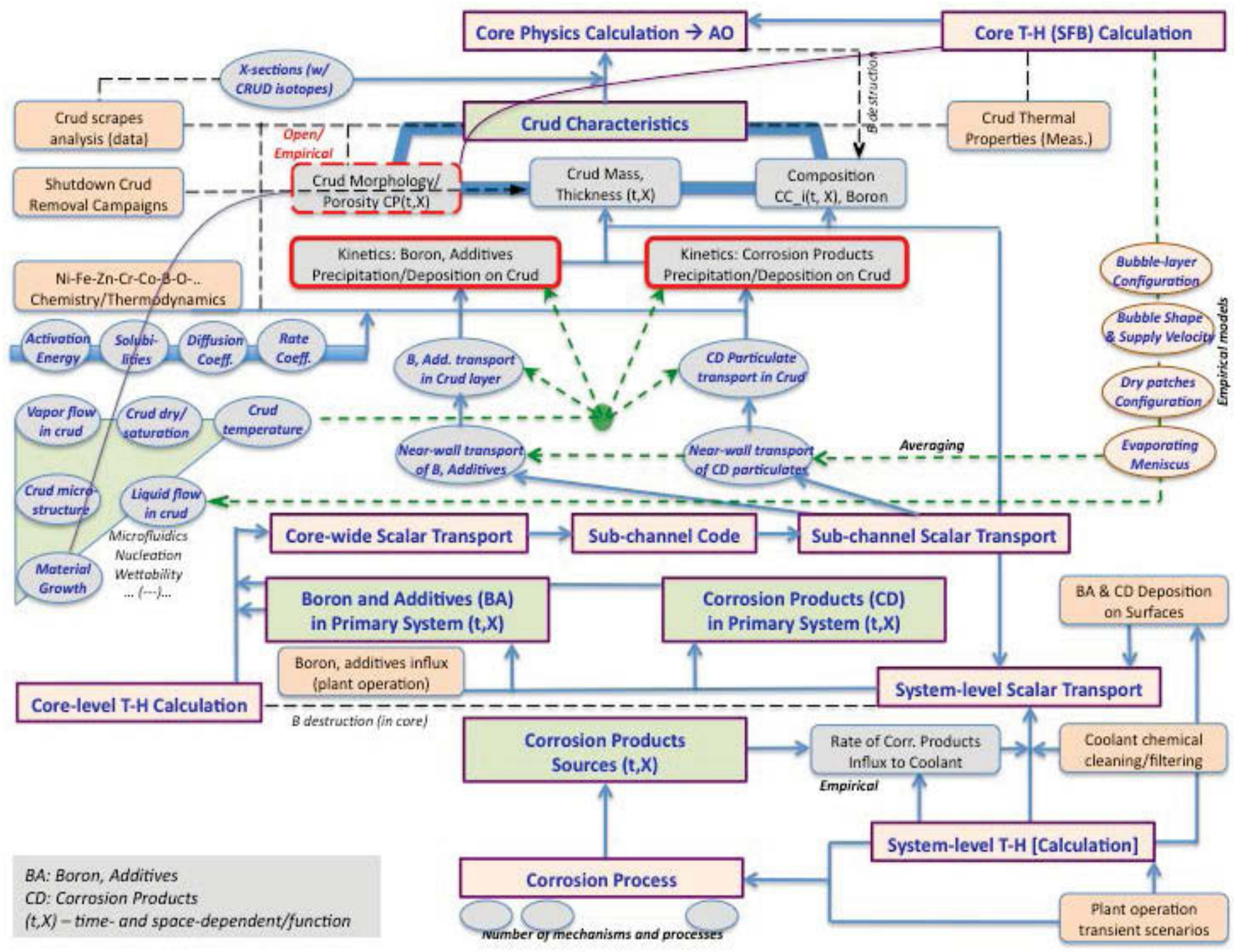

Figure 4.4. CRUD and related coolant chemistry and corrosion products phenomenology, uncertainty sources and propagation ${ }^{26} 27$.

${ }^{26}$ MAMBA modeling covers an upper left part of this framework. 


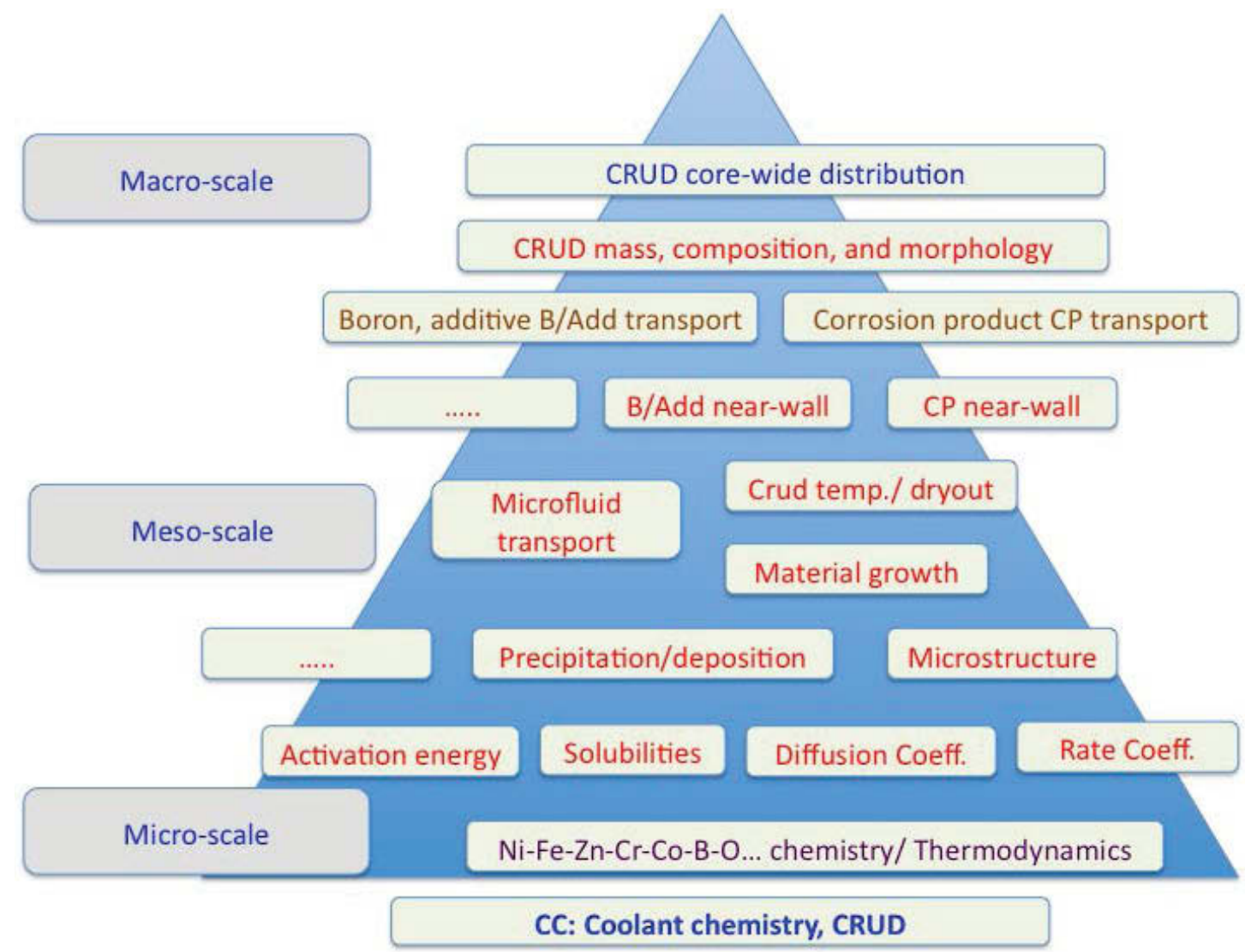

Figure 4.5. CRUD dimension of the CIPS framework (Figure 4.1).

\subsubsection{Thermal-hydraulics — CRUD Coupling}

Interfaces between $\mathrm{TH}$ and $\mathrm{CC}$ are shown in Figure 4.4 (for coolant chemistry/ CRUD decomposition) to occur at all levels. Near-wall transport and resulting distribution (residence time, saturation) of chemicals (Boron, additives) and corrosion products are governed by fluid flow patterns in the wall-region boiling layer. The liquid coolant also enters porous structures of the crud, affecting precipitation/deposition of crud materials and crud temperature field. Near-wall transport and near-wall distribution (concentration) of corrosion products, particulates, and soluble chemicals (Boron, additives), will have a direct influence over the precipitation, deposition and crud growth. Theoretically, the higher the concentration is, the larger the precipitation/ deposition. A limiting case is when the concentration reaches saturation level.

27 Detailed discussion of physics and sources of uncertainty related to CRUD interface with TH (SFB) is provided in a separate report (VUQ.VVDA.P05.4). 
Also on Figure 4.4, "crud morphology/porosity" is shown to exert influence on SFB. Mechanisms of this $\mathrm{CC} \rightarrow \mathrm{TH}$ feedback (on nucleation, nucleation pattern, wettability) can be seen in Figure 4.2 via lines emanated from boxes representing "surface morphology" and "coolant chemistry" factors.

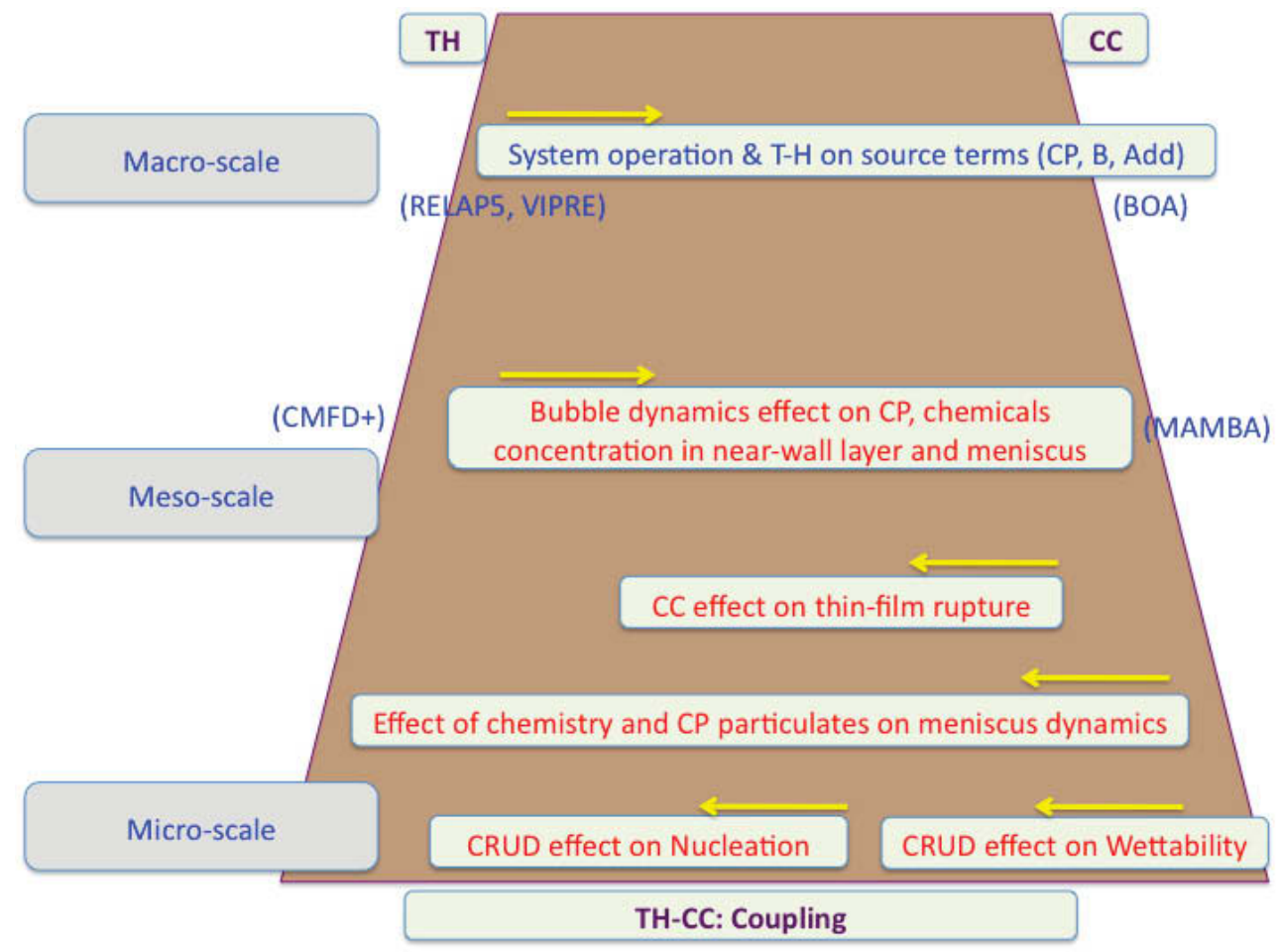

Figure 4.4. Thermal-hydraulics (TH) and Coolant chemistry/CRUD (CC) coupling. Arrows show the direction of information. Micro-scale interactions are dominated by $\mathrm{CC} \rightarrow \mathrm{TH}$ direction (as crud morphology and composition affect nucleation and wettability). Meso-scale and macro-scale interactions are dominated by $\mathrm{TH} \rightarrow \mathrm{CC}$ direction (as fluid flow governs transport of chemicals and particulate materials to the near-wall region). Modeling of fluid flow characteristics needed for describing the $\mathrm{TH} \rightarrow \mathrm{CC}$ feedback requires substantial revision/adaptation/enhancement of CMFD (thus, $C M F D+)$. The red font boxes are indicative of lack of data to quantify modeling assumptions about the interactions and calibrate corresponding models.

While interfaces are provided between tools developed by MPO and THM (and others), the modeling would have to be highly heuristic because of limited expertise in complex multiphase dynamics in the near-wall bubbly layer. Absence of data hampers effort to assess and reduce uncertainty in the interfacing models. 
Consider Path [A] (Sub-section 2.5.3) with single-phase CFD. This option would assume that particulates and chemicals are uniformly distributed in the flow channel, validly in a well-mixed turbulent flow. When boiling is considered (but not modeled), a simplistic treatment would use a maximum deposition rate that is product of the (channel-average) concentration and the boiling rate (i.e. assuming boiling-at-the-bottom-and-condensing-on-theupper-dome bubble is an ideal distillation machine so all chemicals/particulates become crud). Actual deposition is far below that rate.

A more realistic modeling of crud needs input from THM, e.g., concentration of chemicals and particles in the near-wall fluid layer, and deposition rate. As boiling occurs, evaporating fluid meniscus layer (beneath the bubble) gets increasingly enriched in chemicals. If the bubble stays long enough on the surface, the triple contact line (of the meniscus) will get oversaturated with chemicals and particulates. However, if the nucleated bubbles grow to certain size, then detach and slide away, their meniscus does not have enough residence/evaporation time for concentrating the chemicals toward saturation. This shows the importance of bubble departure (diameter, frequency) in a condensing turbulent flow, and this all in turns depends on contact line dynamics, nucleation phenomena/patterns, that all in turns depends on surface material/morphological conditions (oxidation, deposits, crud).

Determining concentration of chemicals and particulates in near-wall layer isn't simple for boiling situation. The velocity field that would be calculated from two-fluid models (assuming that it can be validated), is averaged-in-time and in space. This "effective field" velocity in the normal-to-the-wall direction is small and can hardly be predicted (there were void distribution and some data on axial velocity profile, but never radial near-wall fluid velocity in boiling flow). Thus, for the problem at hand, time-resolved boundary-layer velocity and fluid/particle residence time are needed.

This presents a source of uncertainty that even a well-calibrated two-phase (six-equation, with k-epsilon turbulence) "effective-field" model cannot help reduce. At the same time, description of details of thermal-fluid and transport in boundary layers introduce even more models, more parameters and hence uncertainties. It requires more research (including well-planned ITM simulation) and carefully designed experiments before the uncertainty can be brought down. The choices are: (i) keeping the approximations, and essentially hitting on the uncertainty plateau, and (ii) refining the modeling framework (for near-wall $\mathrm{T}-\mathrm{H}$ ) with a chance to bring the uncertainty to a lower plateau.

\subsubsection{Other technical areas and couplings}

RT-CC (due to boron), RT-TH (due to void moderation), and RT-Fuels (due to fuel temperature) are strong couplings. Spatially, RT-CC-TH are trio 
coupling over crud location, where boron and boiling induce each other and co-present.

For core neutronics, the hierarchy is comprised of three levels: crosssections (boron/crud effect), radiation transport (pin-resolved with $\mathrm{TH}$ and crud's boron feedback) and core-wide neutron diffusion.

Recommendation 4.1: Further elaborate and document the hierarchy for CIPS. Use the hierarchy to characterize validation data content.

\subsection{CIPS Validation Data Plan: Update}

\subsubsection{CIPS Predictability}

Constituting the CIPS challenge problem's objective, "predictability" here refers to the use of CASL-developed advanced simulation capability to enable:

- sound physics-based modeling and capturing of key governing processes;

- a robust capability to calculate CIPS QOIs;

- providing quantitative assessment of the calculations;

- confidence that axial offset is calculated within 3\% for operating plants;

- obtaining good comparison with plant measured data for the right reason.

With VERA-BL tools, the codes are based on coarse-grain models that have been developed and refined over decades, and reached a fair maturity. Yet, "predictability" has been also elusive with VERA-BL tools (and that motivates the CASL work scope). There is a substantial body of experience, insights and data that went into the models and their relations. Untangling them for UQ would cause the same situation denoted in (i) for VERA-AC. Additional difficulty in performing extensive UQ work by the larger CASL community is associated with the proprietary nature of industry's and commercial codes.

With VERA-AC tools, the analysis performed and summarized in preceding sections of this report suggests that this "predictability" is elusive in practice. VERA-AC tools, by themselves, are not expected to become capable of delivering the CIPS predictions within CASL Phase I. 
The principal constraints for VERA-AC capability set are:

(i) there are a large number of physics models, for which CIPS-conditionsrelevant micro-/meso-scale data necessary for calibration do not exist (and are not expected to become available in next 3-5 years); consequently, this implies high uncertainty in VERA-AC prediction for CIPS conditions;

(ii) (assuming CASL overcome the above constraint) VERA-AC tools, once calibrated, provide high-fidelity simulation for a group of phenomena (CRUD, subcooled boiling). However, there are areas (e.g., near-wall transport of chemicals and corrosion products) that are not treated with the same level of fidelity or robustness. Also due to their novelty, these areas lack data and observations. Thus, they present weaker links (due to modeling used in those areas) and drive the uncertainty in the CIPS prediction.

(iii) (assuming CASL overcome both above constraints) VERA-AC highfidelity/high-resolution simulation tools are computationally too demanding that it is not possible to use them for CIPS predictions (yearslong operational transients; core-wide analysis) and many runs needed for UQ. Logically these AC tools need to be coupled with middle-fidelity simulation (e.g., BL) tools. Thus, the uncertainty in middle-fidelity tools and uncertainty due to cross-scale integration (conditioning/homogenization models) would drive the quality of CIPS predictions. There is a critical need for new experiments that examine these cross-scale models.

In all three instances (i-iii), the need for "validation data" figures eminently.

Fundamentally (and mathematically), elusiveness of "CIPS predictability" stems from the "determinism", upon which VERA tools are developed. The modeling that went into VERA foundation is mechanistic. The underlying conservation laws rule in the form of partial differential equations (PDE), whose validity is vetted at infinitesimality. Much of observable processes and measurable parameters are at finite, and even sizable scales. Within this scale is the infinity of randomness, which must be homogenized to fit the PDE mind set. This homogenization and resulting "closure relations" are born out of deterministic world's deficiency, and hence inherently uncertain, an aleatory and epistemic mixing. The cure is to assign an uncertainty distribution to it. Thus, in the mechanistic realm (ruled by PDE) uncertainty is an afterthought! Much in alignment with "uncertainty principle", the higher resolution one pushes simulation in one dimension (physics), the higher the chance is that uncertainty skyrockets in other dimensions. 
Practically (and physically), elusiveness of "CIPS predictability" lies with stochastic and delicate nature of governing physics, such as nucleation (Dinh and Theofanous, 2003). In fact, once a vapor bubble nucleated on cladding surface, and stays on it, it serves as an ideal desalination/ distillation machine. Boron, chemicals and particulate corrosion products become saturated in evaporating meniscus layer beneath that standing bubble, precipitated and deposited on cladding surface. This further reduces nucleation energy barrier, triggering a chain reaction for further crud formation and buildup. Thus, how the process is initiated (nucleation) is critical. Can we predict nucleation deterministically? It has been shown (e.g., in Theofanous et al., 2002, Dinh et al, 2003, Dinh and Theofanous, 2004) that the traditional text-book view of boiling (including vapor bubble nucleation) misled generations. Surface micro-cavity of certain size is not needed for bubble nucleation as one was taught to believe. All that needed are nanoscopic Frenkel's islands of inhomogeneity to significantly reduce the nucleation energy barrier (Fig. 4.5).

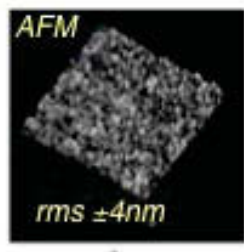

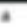
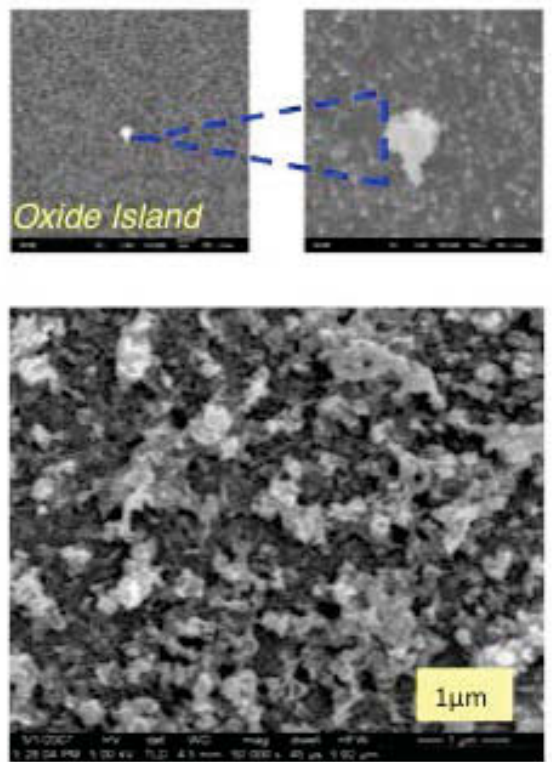
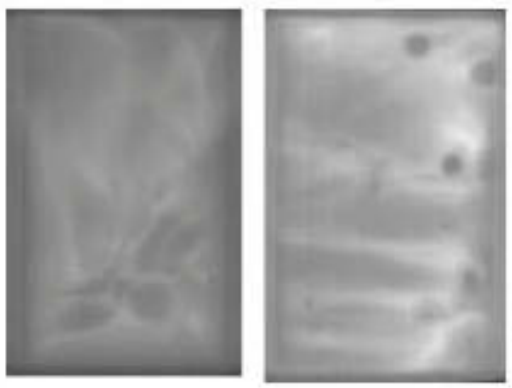

Fresh smooth surface

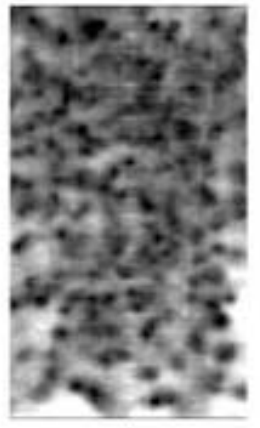

a)

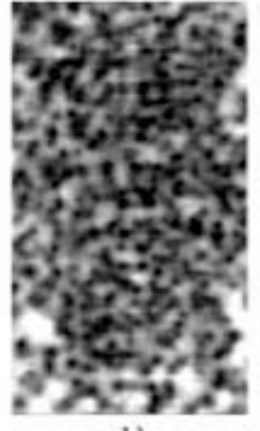

b)
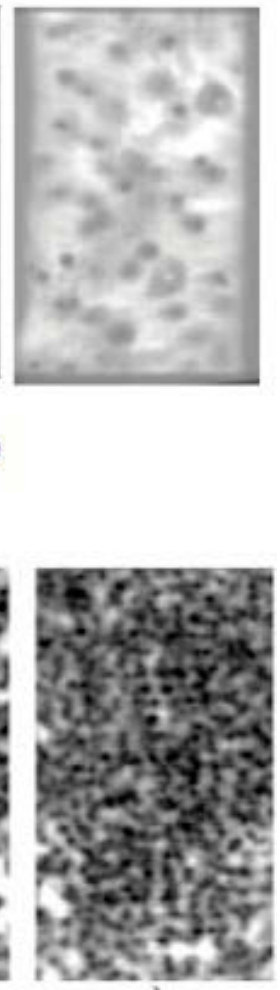

c)
Oxidized surface

Figure 4.5. Surface nano-morphology and material inhomogeneity are central role in nucleation (Theofanous et al., 2002; Dinh et al; 2003; Dinh and Theofanous, 2004). Surface oxidation and defects significantly lower the energy barrier (surface superheat) required for nucleation. Deposits of certain materials and microstructures influence nucleation energy barriers more than that of other materials/microstructures. 
Left column: AFM and SEM images of heater surface that is nanoscopically smooth and fresh, surface with inceptive oxidation, and heavily oxidized (aged) surface.

Right column: infrared thermometry images. Temperature is scaled with brightness. Dark spots are bubble foorprints. Aged/oxidized surface has high nucleation site density even though surface superheat is substantially lower.

In fact, it is not the "island" but its "borderline" that matters. Relevant to this discussion is that surface condition contributes as much to nucleation as surface's thermal condition (heat flux). Local defect on cladding surface occurred in fuel manufacturing, transport, or installation (e.g., grid spacerinduced scratches), would trigger nucleation in areas where surface temperature superheat (over saturation) is small and coolant is still deeply sub-cooled; see SDk in Figure 4.6. This deep sub-cooling helps "hold" the nucleated bubble small and attached to the surface for a longer period, which promotes accumulation of potential deposits at the bubble's triple contact line; for more discussion, see Sections 4.1.2 and 4.1.4.

Thus, both mathematically and physically, deterministic framework (with uncertainty as afterthought) is not conducive for analysis of complex phenomena, like CIPS. In practical term, this "determinism" can become counter-productive, excessively conservative in one case, and ineffectively protective in another.

For example, given observation of crud formation in a plant, and assuming that one has tools to backup the conditions in the reactor core, the crud evidence is used to calibrate the model of crud formation. However, neither state of cladding surface of the crudded rods prior to their loading into the core is known, nor knowledge about other stochastic factors is available. Consequently, the cladding surface conditions are assumed to be "core average", or "load average", ignoring the very possibility that surface defects (scratches) are local conditions. Models calibrated on "core average" conditions on data found on local area necessarily lead to excessive conservatism in the prediction of formation of crud (typically, over a substantial faction of core surface area). Not only that such predictions would not be confirmed by observations in a next core load, or next reactor, they also misinform plant operation and safety. 


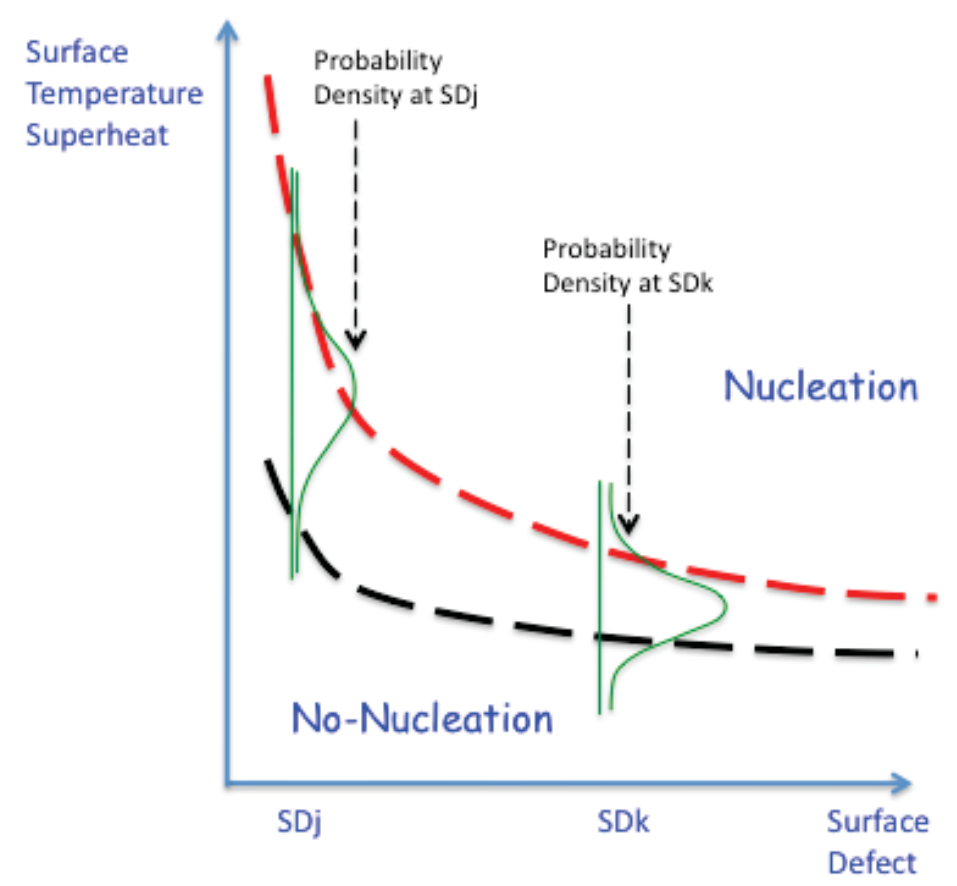

Figure 4.6. Nucleation threshold. Surface defect (SD) condition can initiate nucleation under much-lowered surface superheat temperature (SST). The Causal Relation CR (SST--SD) is probabilistic physics-based (see also Figure 4.7). Cladding surface defect SD itself must be represented probabilistically due to uncontrolled variability. SST is also probabilistic, to account for uncontrolled factors, including anomaly in fuel pellet thermo-mechanics and pellet-cladding interactions, asymmetric swelling and interaction with cladding, and conjugate heat transfer.

The elusive variability of CIPS has caused experts' wondering about apparent unpredictability of the phenomenon: crud was found to occur in one of presumably identical cores, in one of apparently identical fuel assemblies, identical fuel rods, and only on one side of the fuel rods; remarkably but now understandably, crud was found to occur not in hottest rods. Now, recognizing inherent multi-dimensional variability e.g., in cladding surface conditions, and operating conditions, it appears futile to use deterministic predictions to bruteforcedly capture those details.

However, one can argue that the CIPS problem may become predictably solvable if the issue is approached in a probabilistic/deterministic framework. Such risk-informed approach ${ }^{28}$ is trendy and proven useful in dealing with

28 The premise of a probabilistic (risk) assessment is to recognize that as plant operators thrive for economics (power uprate), there will always be a non-zero probability for failures with bad outcome (e.g., crud growth on some fuel claddings in the case of CIPS); see Figure 3.1. The practical question is how small should this 
complex issues, where both epistemic uncertainty and aleatory uncertainty (variability) are large. In severe nuclear reactor accident safety, the approach was pioneered in the work of Professor T.G. Theofanous (UCSB) through a so-called Risk-Oriented Accident Analysis Methodology (ROAAM). The methodology was used to successfully resolve several major safety issues in existing LWRs and ALWR designs. The latest example of ROAAM application is documented in the work of Theofanous and Dinh (2008) for ESBWR.

It is instructive to note that the probabilistic/deterministic solution framework invariably influences the way observations and data are collected, processed and used. For example, observed crud in a reactor core should not be used to conveniently and deterministically calibrate, in isolation, a boilingdriven crud formation model ${ }^{29}$. Within a Bayesian inference framework, the evidence should correspondingly trace its root causes through all probabilistic contributors.

failure probability $\mathrm{P}$ be for it to be acceptable in a given engineering application. The risk-informed answer requires knowledge of expected/calculated consequence $\mathrm{C}$ of the low-probability failure.

29 THM Path [A] (Subsection 2.5.3) uses single-phase CFD/CHT (conjugate heat transfer) results to determine boiling surface area. Such model-form oversimplifications ignore microphysical nature of boiling ("throw the baby out with the bath water"), thus responsible for over-prediction of crud. 


\subsubsection{Probabilistic Integration Framework}

Development of a comprehensive probabilistic integration framework for CIPS problem is beyond the scope of this work. In fact, VERA/CIPS workflow is to be detailed as part of the "CRUD Challenge Problem" Product Integration effort, as discussed in Sections 2.1-2.2.

Several foundational elements for developing and implementing a probabilistic integration framework are noteworthy at this time.

First, and foremost, a goal-oriented framework is built with a finite number of components, which are selected for their potential significant impact (judged on evidences or hypothesis) on the goal. In other words, components are top-down aggregate as opposed to bottom-up inclusive. The first advantage of having such a framework is in greatly simplifying the simulation task. Instead of insurmountable task of providing computations over a total models' and parameters' space, the framework focuses computations on what experts judge to be significant, rendering it more practical. Since the operation is subjective, decomposition requires V\&V.

In practice, decomposition is largely influenced by availability of calculation tools, as well as of data that can be used to support the calculation. In example shown on Figure 4.7, calculation tools are denoted as CR (for Causal Relation) ${ }^{30}$, which take input parameters (some are as probability distributions) and generate output parameters in pdf. In some cases, the CR is provided by AMS capability (VERA-AC). In other cases, it is a data-based probabilistic mapping, like one shown in Figure 4.6. Given an input of SD (surface defect) and SST (surface superheat temperature), the Causal Relation produces nucleation's pdf. The input, SST and SD, are generally probabilistic too. In case of deficient data (as for SD in this case), qualitative knowledge is represented using ROAAM's "Process Likelihood", typically resulting in histogram.

Table 4.2 shows definition of probability levels introduced in ROAAM. For physical processes, this translates experts' knowledge into numerical systems, much in the same way as steps taken for "quantification and integration of heterogeneous data" in Sub-section 3.2.3. Effectively, they enable integrated uncertainty treatment needed for multi-physics multi-scale problems, when very different tools and knowledge bases are brought together for solution.

\footnotetext{
${ }^{30}$ Decomposition and CRs are used consistently with ROAAM terminology. For more discussion, see Theofanous (1996).
} 


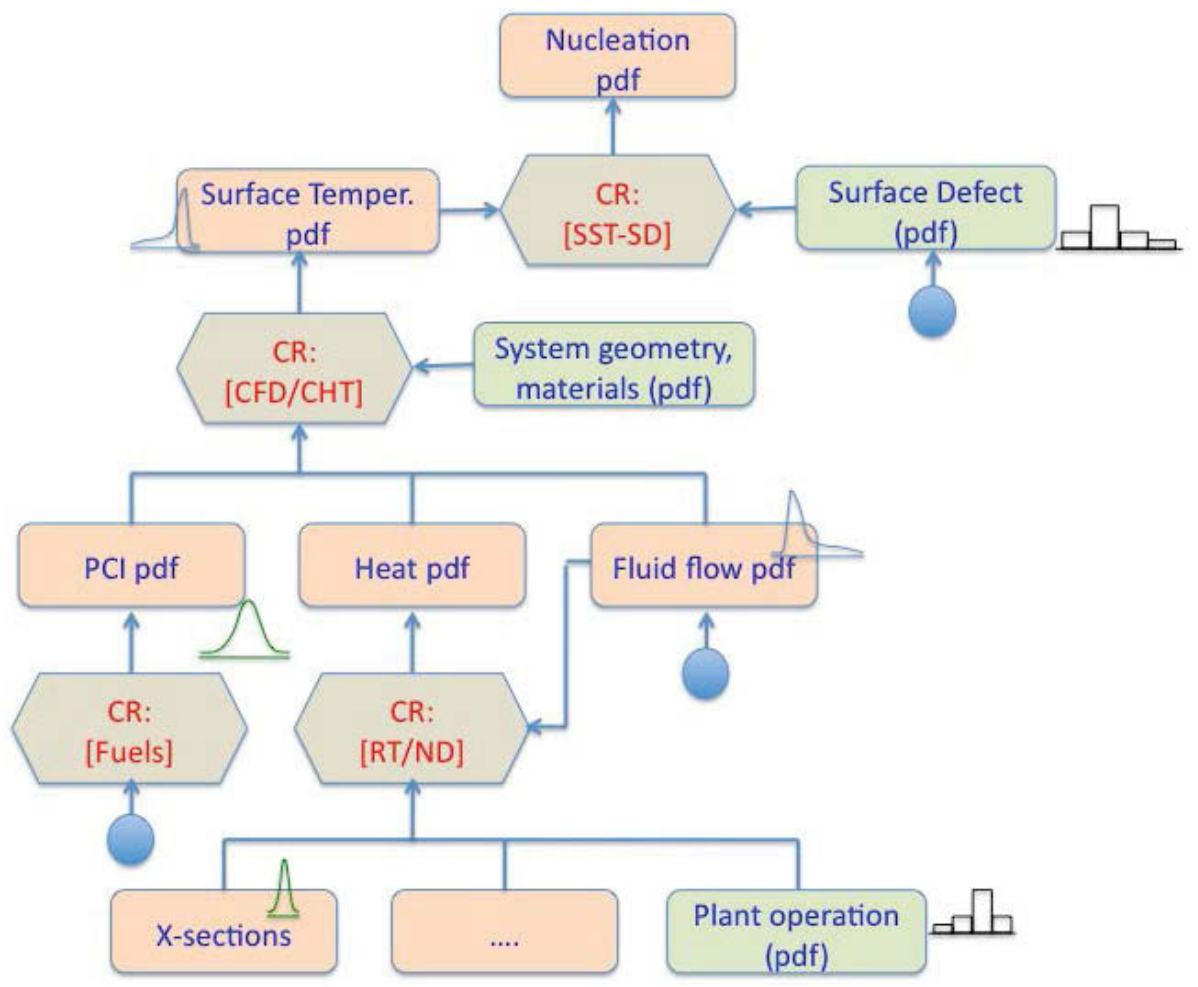

Figure 4.7. Decomposition of a "nucleation" branch in a hypothesized CIPS framework. The circles denote branch for further decomposition. Probability distributions are notional.

Table 4.2. Definition of probability levels (Theofanous, 1996)

\begin{tabular}{|c|l|}
\hline $\begin{array}{c}\text { Process } \\
\text { Likelihood }\end{array}$ & \multicolumn{1}{c|}{ Process Characteristics } \\
\hline $\mathbf{1 / 1 0}$ & $\begin{array}{l}\text { Behavior is within known trends but obtainable only at the } \\
\text { edge-of-spectrum parameters }\end{array}$ \\
\hline $\mathbf{1 / 1 0 0}$ & $\begin{array}{l}\text { Behavior cannot be positively excluded, but it is outside the } \\
\text { spectrum of reason }\end{array}$ \\
\hline $\mathbf{1 / 1 0 0 0}$ & $\begin{array}{l}\text { Behavior is physically unreasonable and violates well-known } \\
\text { reality. Its occurrence can be argued against positively }\end{array}$ \\
\hline
\end{tabular}

Another important advantage of the probabilistic integration is that all kinds of relevant capability (BL, SC, AC-class codes) can be used to produce information deem-useful for uncertainty reduction (narrowing pdf of parameters of interest). Codes are used at the scale they are designed for. 
Confidence in the simulation results is judged, using probability levels. This judgment factors in the quality of validation data support, and how far has the simulation gone beyond the validation domain. Wherever robust and accurate multi-physics coupling and cross-scale integration are available, they are used as one CR (Causal Relation). Where mechanistic treatment of such couplings absent, the probabilistic integration framework still makes use of the tools through a rudimentary CR (such as data-based probabilistic mapping in [CR: SST-SD]; Figure 4.6) for their coupling, and assigns appropriate level of confidence to the process and result.

\section{Recommendation 4.2: Develop and demonstrate a probabilistic framework for addressing select hypotheses about CIPS.}

\subsubsection{Validation Data Plan Implementation: Case Study}

With the outcome of the work performed in POR4 on Validation Data Plan and results of interactions with and input from THM and MPO on validation data plans for advanced capability in the CRUD problems, there is a consensus that (i) basic positions/principles of VDP and recommendations for an application-oriented Bayesian data assimilation framework are reasonable and ought to be demonstrated on critical development areas in CASL (e.g., THM-CMFD-SFB) and that (ii) subcooled flow boiling (SFB) is an area where a systematic VUQ-guided approach to model assessment, model calibration and code validation is much needed. This led to suggestion of using SFB as a case study for implementation of VDP.

The task objective is to enable implementation of an application-oriented, VUQ-guided validation data support strategy whose key positions are outlined in this VUQ-VVDA.P4.02 milestone report. The task in POR5 uses a test case to focus development, testing and application of the proposed strategy and associated infrastructure for validation data support. The selected test case is an important capability, whose development has been hampered by validation data challenges. Namely, the case study is the validation support for advanced CFD-based capability for predicting subcooled flow boiling (SFB) two-phase thermal-hydraulics, including thermal-hydraulics/material-chemistry interface, in crud challenge problems.

The case study's technical approach includes the following steps:

1. Review modeling approaches, data bases and simulation capability for crud-related thermal-hydraulics and material-chemistry processes in subcooled flow boiling (SFB) at all relevant scales. 
2. Identify, assess and treat various sources of uncertainty in SFB experimental data related to their application in a PWR crud analysis.

3. Evaluate sub-cooled flow boiling models and their hierarchical decomposition for modeling/ experimentation/ validation consistency.

4. Design, initial implementation and application of a framework (including infrastructure) for SFB model calibration and validation.

5. Document the implementation of SFB thermal-hydraulics validation data plan, lessons learned, and recommendations for improvements.

6. The work is to be performed in close collaboration with THM, MPO, and AMA experts, and with the CASL Data Center initiative.

The work supports, and benefit from, activity in cross-FA Working Groups on VUQ and Validation Data, which provide input on SFB data qualification, uncertainty quantification and validation database infrastructure.

Also a proposal is made to initiate the design, implementation, and demonstration of the CASL Data Center (CDC) in PoR-5. Along with the VDP study detailed in this report, the CDC is instrumental to the "Validation Data" Product Integration (VDPI) that is envisioned by the CASL SLT ${ }^{31}$.

${ }^{31}$ CASL PoR-5 planning meting (communication, P. Turinsky, March 13, 2012). 


\section{CONSLUSIONS AND RECOMMENDATIONS}

This report documents the analysis, findings, and recommendations resulting from a study of the CIPS validation data plan. The VDP effort aims to establish a technical basis and framework for guiding the validation data product integration. It is motivated by the increasingly pressing question: how to deal with deficient data support in an AMS program like CASL? The technical approach taken in the present study of VDP includes (a) a VDP component review, (b) an integrated assessment, (c) revisiting mission/strategy (in part to ensure consistency in modeling-experimentationvalidation, and (d) updating the VDP with specification of tasks in all components.

While an AMS project characteristically starts with enthusiasm about the role of super-computing power in enabling high-resolution/high-fidelity simulations, sooner rather than later, the project execution runs into several major roadblocks. The first is recognition of how far (or how short) one can take "ab initio", "first-principle" simulations into really complex (e.g., multiphysics, multi-scale) phenomena or engineering applications. There is no computing power in the foreseeable future that can bridge this gap. Beyond a narrow domain where direct numerical simulations adequately capture reality, practical analyses always require modeling. The closer a simulation comes to being useful in complex engineering applications, the more critical modeling becomes. As a rule, this leads to the recognition that experimental data are deficient for the purpose of calibrating and validating increasingly complex and sensitive models embedded in high-fidelity simulations. Last but not least, the development and developmental assessment of AMS codes are both timeand resource-intensive, often far more than initially planned.

CASL possesses and is developing an impressive set of capabilities, including state-of-the-practice and state-of-the-art AMS codes. Information flow has started on a two-way street: (1) applications of industry codes give researchers/modelers important insights and (2) results from high-resolution simulations on supercomputers, admittedly snapshots at this time, provide engineers and analysts with insights not previously available. In other words, the activities contribute to reducing epistemic, largely model-form uncertainty, the benefit of which cannot be not easily quantified. In fact, the situation delineates that a part of the challenge is in communications. More broadly, validation is to communicate confidence in the predictive capability, for whose "case" all relevant evidences and technical arguments matter. 
The time is ripe and critical for CASL to establish a comprehensive workflow for solving the CIPS challenge problem within the constraints that CASL is in. Such an application-oriented solution framework is instrumental for the planning of the development and assessment of the CIPS-related simulation capability. The framework will help define the CIPS-specific validation requirements, validation metrics, and validation plan. It will also help guide and coordinate efforts in benchmarks and, ultimately, the validation data support.

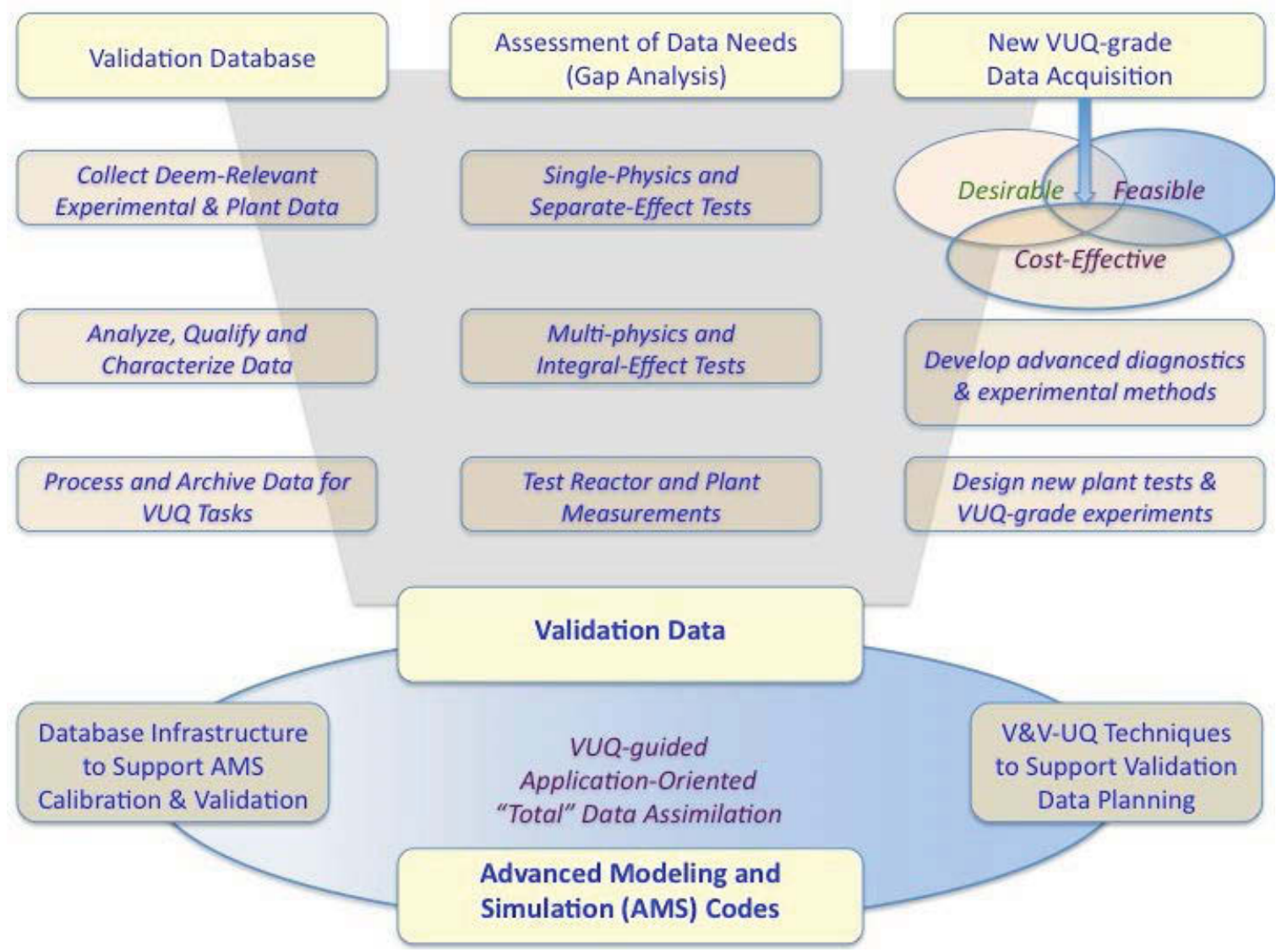

Figure 5.1. An update overview of validation data activity to be conducted in tight integration with AMS capability (both VERA-BL and VERA-AC), $V U Q$ techniques and development of database infrastructure via CDC (see Figure 1.1 for comparison).

The study also suggests that CASL adopts a pragmatic (applicationoriented) and holistic (communication) approach to validation and validation data. This means bringing to bear evidences and data of all types and all origins, developing infrastructure to house the data, developing methods for qualification and consistent treatment of the heterogeneous data, and developing methods for extracting more value from available experiments and datasets. In the proposed VDP, VERA simulation capability, validation 
activity, data, and data support activity are tightly coupled through a CASL Data Center (see Figure 5.1). The plan also includes a VUQ-guided use of code-to-code cross fertilization and a probabilistic integration approach. Such an approach can enable CASL to take advantages of the available capability set, overcoming limitations associated with immaturity of more mechanistic methods for multi-scale and multi-physics integration.

The approach proposed in this study can be embodied in the following technical recommendations for the VDP (Table 5.1).

Table 5.1. Summary of technical recommendations for CASL and CIPS VDP

\begin{tabular}{|c|c|}
\hline 2.1 & $\begin{array}{l}\text { Establish the uncertainty of CIPS prediction by VERA-BL capability. } \\
\text { This baselining is critical for measuring progress in VERA-AC } \\
\text { development, and for strategic planning including VDP. }\end{array}$ \\
\hline 2.2 & $\begin{array}{l}\text { Establish a practical, and scrutable workflow for applying VERA } \\
\text { codes for CIPS challenge problem solving. }\end{array}$ \\
\hline 2.3 & $\begin{array}{l}\text { Establish and prioritize CIPS-specific requirements for integrated } \\
\text { capability and for each component, to guide development and } \\
\text { assessment of VERA components. }\end{array}$ \\
\hline 2.4 & Establish a CIPS-specific integrated calibration and validation plan. \\
\hline 2.5 & $\begin{array}{l}\text { Establish a CASL-wide policy, process and infrastructure on } \\
\text { validation data management, including inventory, warehousing, } \\
\text { processing, and controlled secure access. }\end{array}$ \\
\hline 2.6 & $\begin{array}{l}\text { Develop "gap-tooth" scheme for integration of VERA-BL (e.g., } \\
\text { neuron diffusion) capability for long-transient simulation and VERA- } \\
\text { AC (e.g., radiation transport) capability for high-resolution snapshots } \\
\text { simulation. }\end{array}$ \\
\hline 2.7 & benchmarks for multi-scale "gap-tooth" integration. \\
\hline 2.8 & $\begin{array}{l}\text { Perform VUQ-grade experiments (two-phase flow, SFB) and analysis } \\
\text { to establish impact of assumptions, maturity, and usefulness of Paths } \\
{[\mathrm{A}] \text { and [B]. }}\end{array}$ \\
\hline 2.9 & $\begin{array}{l}\text { Create a VERA-wide list of models, modeling assumptions, and } \\
\text { model parameters. Each validation task must document } \\
\text { models/assumptions/parameters scrutinized in the benchmark and } \\
\text { additional assumptions made in order to implement the } \\
\text { benchmarks. }\end{array}$ \\
\hline 2.10 & $\begin{array}{l}\text { Develop validation hierarchy that reflects specificity of multi-physics, } \\
\text { multi-scale, multi-component nature of CIPS problem. }\end{array}$ \\
\hline 3.1 & Establish a risk-informed margin framework for CIPS. \\
\hline 3.2 & ASL \\
\hline
\end{tabular}




\begin{tabular}{|l|l|}
\hline 3.3 & $\begin{array}{l}\text { The CDC design and an initial implementation focus on creating data } \\
\text { functions, which support a high-priority development in CASL. }\end{array}$ \\
\hline 3.4 & $\begin{array}{l}\text { Based on knowledge base of CIPS-related phenomena, codes, } \\
\text { experiments, and plant data, develop a CIPS-oriented Bayesian } \\
\text { calibration framework, test and demonstrate its performance on } \\
\text { surrogate models of multi-physics multi-scale simulation capability. }\end{array}$ \\
\hline 3.5 & $\begin{array}{l}\text { Investigate options and consequences of grading and weighting } \\
\text { systems on model calibration and uncertainty analysis. }\end{array}$ \\
\hline 3.6 & $\begin{array}{l}\text { Develop and implement a plan for using VERA-BL-generated } \\
\text { numerical experiments for aiding developmental assessment of select } \\
\text { VERA-AC tools, and their multi-physics capability. }\end{array}$ \\
\hline 4.1 & $\begin{array}{l}\text { Further elaborate and document the hierarchy for CIPS. Use the } \\
\text { hierarchy to characterize validation data content. }\end{array}$ \\
\hline 4.2 & $\begin{array}{l}\text { Develop and demonstrate a probabilistic framework for addressing } \\
\text { select hypotheses about CIPS. }\end{array}$ \\
\hline
\end{tabular}

With respect to validation support for AMS, other recommendations that span across the DOE-NE R\&D programs are:

(i) Promoting a culture change through development, demonstration and broad use of a structured, holistic and quantitative approach to validation of advanced modeling and simulation codes.

(ii) Encouraging and enabling cross-programs sharing on efforts in addressing common data needs, and in establishing common standards, processes and infrastructure for acquisition, qualification, archiving, mining and usage of validation data.

(iii) Supporting a nation-wide coordinated infrastructure network of facilities and databases. This includes a broad range of experimental facilities for separate-effect testing (primarily at universities) and integral-effect testing in facilities at both the industry vendors and select universities.

(iv) Supporting national laboratory-based facilities that address critical gaps but require a high level of science and technology support beyond the scope of university and vendor environments. In particular, these facilities will focus on capability to develop data for validation of multi-physics AMS.

(v) Promoting validation in international collaboration. The US community, particularly CASL, contributes pioneering advances in VUQ methods. Other countries provide access to validation experiments planned in their facilities and data from select validation tests and observations conducted in plants. 


\section{BIBLIOGRAPHY ${ }^{32}$}

AIAA. Guide for the Verification and Validation of Computational Fluid Dynamics Simulations, American Institute of Aeronautics and Astronautics, AIAA-G-0771998, Reston, VA, 1998.

A.-M. Alvarez, Studsvik Cladding Integrity Programme, Touch Briefings [Nuclear Energy Review], 2007. Presentation, 2009.

C. Anghel, A.-M. Alvarez Holston, G. Lysell, et al., "An Out-of-Pile Method to Investigate Iodine-induced SCC of Irradiated Cladding”, LWR Fuel Performance Meeting, September 6-9, 2009, Paris, France.

ANS. Guidelines for the Verification and Validation of Scientific and Engineering Computer Programs for the Nuclear Industry, American Nuclear Society, ANSI/ANS-10.4-1987, 1987.

ASME Guide for Verification and Validation in Computational Solid Mechanics, The American Society of Mechanical Engineers, ISBN \#: 079183042X, 2006.

ASME V\&V 20 - 2009 Standard for Verification and Validation in Computational Fluid Dynamics and Heat Transfer, 2009.

Bang, I.C., Chang, S.H., Baek, W.P., 2004, Visualisation of the subcooled flow boiling of R-134a in a vertical rectangular channel with an electrically heated wall, Int. J. Heat Mass Transfer, 47, 4349-4363.

G.G. Bartolomei, V.M. Chanturiya, "Experimental study of true void fraction when boiling subcooled water in vertical tubes", Thermal Engineering 14, 1967, pp. 123128, translated from Teploenergetika 14, 1967, pp. 80-83.

Basu, N., Warrier, G.R., Dhir, V., 2002, Onset of Nucleate Boling and Active Nucleation Site Density during Subcooled Flow Boling, J. Heat Transfer, 124, 717728.

Basu, N., Warrier, G.R., Dhir, V., 2005a, Wall Heat Flux Partitioning during Subcooled Flow Boiling: Part 1 - Model Development, J. Heat Transfer, 127, 131140 .

Basu, N., Warrier, G.R., Dhir, V., 2005b, Wall Heat Flux Partitioning during Subcooled Flow Boiling: Part 2 - Model Validation, J. Heat Transfer, 127, 131-140.

\footnotetext{
${ }^{32}$ Including references cited, used and recommended for the topic of this report.
} 
P. Bennett, B. Beverskog, and R. Suther (J. Deshon). "Halden In-Reactor Test to Exhibit PWR Axial Offset Anomaly”, EPRI-1008106, 120p., December 2004

P. Bennett, B. Beverskog, and R. Suther (J. Deshon). "Demonstration of the PWR AOA in the Halden Reactor", IFE, Norway, 2007,

P. Bennett, , "Effects of water chemistry and thermal-hydraulic conditions on crud formation on PWR fuel in the Halden reactor", IFE, Norway, 2009.

Bertel, M., Ishii, M., Masukawa, T, Mi, Y., Situ, R., 2001, Interfacial area Measurements in Subcooled Flow Boiling, Nucl. Eng. Design, 210, 135-155

Bonjour, J., Clausse, M. and Lallemand, M., 2000, Experimental study of the Coalescence Phenomenon during Nucleate Pool Boling, Exp. Thermal and Fluid Science, 20, 180-187.

B. E. Boyack, I. Catton, R. B. Duffey, P. Griffith, K. R. Katsma, G. S. Lellouche, S. Levy, U. S. Rohatgi, G. E. Wilson, W. Wulff, and N. Zuber, "Quantifying Reactor Safety Margins," papers 1 to 6, Nuclear Engineering and Design, Vol. 119, (1990). (NUREG/CR-5249, USNRC, December 1989)

W. A. Byers, "Pressurized Water Reactor Core Crud Mapping”,18th International Conference in Nuclear Engineering (ICONE18) Paper no. ICONE18-30218 pp. 333339 , May 17-21, 2010 , Xi'an, China

CASL, "Proposal for a Consortium for Advanced Simulation of LWRs", Oak Ride National Laboratory, 2009

Chen, T. and Chung, J.N., 2003, Heat Transfer Effects of Coalescence of Bubbles from Various Site Distributions, Proc. R. Soc. Lond. A, 459, 2497-2527.

Chang, S.H., Bang, I.C. and Baek, W.P., 2002, A Photographic study on the nearwall bubble behaviour in Subcooled Flow Boling, Int. J. Thermal Sciences, 41, 609618

Chatpun, S., Watanabe M., Shoji, M, 2004, Experimental study on Characteristics of Nucleate Boling by the Effects of Cavity Arrangement, Exp. Thermal and Fluid Science, 29, 33-40.

CSAU, "Code Scaling, Applicability, and Uncertainty", US NRC, NUREG/CR-5249 (Boyack et al.), 1989. US NRC Regulatory Guide 1.157 ("Best-Estimate Calculations of ECCS Performance", 1989).

H.W. Coleman, W.G. Steele "Experimentation, Validation, and Uncertainty Analysis for Engineers. John Wiley and Sons, Hoboken, NJ, 2009.

T.N. Dinh, and Theofanous, T.G., "Nucleation Phenomena in Boiling", Multiphase Science and Technology. 15(1-4), pp.349-363, 2003. 
T.N. Dinh, J.P. Tu, A.T. Dinh, T.G. Theofanous, "Nucleation Phenomena in Boiling on Nanoscopically Smooth Surfaces", 41st Aerospace Sciences Meeting, Reno, NV, Jan 2003. AIAA-2003-1035.

T.N. Dinh, J.P. Tu and T.G. Theofanous, "Hydrodynamic and Physico-Chemical Nature of Burnout in Pool Boiling", International Conference on Multiphase Flow, Yokohama, Japan, May 2004. Paper 296. 14p.

T.N. Dinh and J.P. Tu, "The Micro-Hydrodynamics that Govern Critical Heat Flux in Pool Boiling", International Conference on Multiphase Flow, Leipzig, Germany, July, 2007.

T.N. Dinh and J.P. Tu, "The Micro-Hydrodynamics that Govern Critical Heat Flux in Pool Boiling", International Conference on Multiphase Flow, Leipzig, Germany, July, 2007.

T.-N. Dinh, V.A. Mousseau, R.R. Nourgaliev and R.W. Youngblood "A Next Generation of Nuclear Plant System Analysis Codes to Support Risk-Informed Safety Margin Characterization," Proceedings of the 13th International Topical Meeting on Nuclear Reactor Thermal Hydraulics (NURETH13), Kanazawa, Ishikawa-Ken, Japan, (2009).

N. Dinh, "CASL Validation Data: An Initial Review", Idaho National laboratory, INL/EXT-11-21017, January 2011, 114p.

EMDAP, "Evaluation Model Development and Assessment Process", US NRC Regulatory Guide 1.203, December 2005 ("Transient and Accident Analysis Methods")

EPRI, "Crud Deposition Studies in the Cirene Loop", (S. Yagnik), Technical Report 1000900, November 2000.

EPRI, "PWR Axial Offset Anomaly Guidelines", Technical Report 1003213, Oct. 2003.

EPRI, "New Experimental Studies of Thermal-hydraulics of Rod Bundles (NESTOR)”, Technical Report 1013424, December 2006.

EPRI, "New Experimental Studies of Thermal-hydraulics of Rod Bundles (NESTOR)", Technical Report 1019423, August 2009.

EPRI, "New Experimental Studies of Thermal-hydraulics of Rod Bundles: Generic Analysis of OMEGA Data", Technical Report 1021039, October 2010.

EPRI, "Assessment of Experimental Data to Support Computational Fluid Dynamics Analysis of PWR Rod Bundle Heat Transfer Studies", Technical Report 1021040, Aug 2010.

EPRI, "Rod Bundle Heat Transfer for PWRs at Operating Conditions", Technical Report 1000215, 2000. 
EPRI, "Subcooled Boiling Data from Rod Bundles", Technical Report 1003383, Sep 2002 (also Karoutas et al, 2004)

S. Ferson and W.L. Oberkampf, "Validation of imprecise probability models", International Journal of Reliability and Safety, V.3 (N.1-3), 2009, p.3-22.

Garnier, J., Manon, E. and Cubizolles, G., 2001, Local Measurements on Flow Boling of Refrigerant 12 in a Vertical Tube, Multiphase Science and Technology, 13, $1-111$.

S. Gong, W. Ma, and T.N. Dinh, "Diagnostic techniques for the dynamics of a thin liquid film under forced flow and evaporating conditions "J. Microfluidics and Nanofludics, Vol.9, N.6, 1077-1089, 2010.

S. Gong, W. Ma, and T.N. Dinh, "An experimental study of rupture dynamics of evaporating liquid film on different heater surfaces", International Journal of Heat and Mass Transfer, 2010

J.M. Hawkes, "The Simulation and Study of Conditions Leading to Axial Offset Anomaly in Pressurized Water Reactors", PhD Thesis, Georgia Institute of Technology December, 2004

J. Henshaw, J.C. McGurk, H.E. Sims, A. Tuson, S. Dickinson, J. Deshon, “A model of chemistry and thermal hydraulics in PWR fuel crud deposits", Journal of Nuclear Materials 353 (2006) 1-11.

S. Hess, R. Schmidt, et al. "VERA Requirements", CASL VRD Working Group (communications), March 2012.

Hibiki, T., Ishii, M., 2003, Active Nucleation Site Density in Boiling Systems, Int. J. Heat Mass Transfer, 46, 2587-2601.

Hibiki, T., Mi, Y., Situ, R., Ishii, M., 2003, Interfacial Area Transport of Vertical Upward Bubbly Two-Phase Flow in an Annulus, Int. J. Heat Mass Transfer, 46, 4949-4962.

D. Higdon, M. Kennedy, J. C. Cavendish, J. A. Cafeo, R. D. Ryne, "Combining Field Data and Computer Simulations for Calibration and Prediction," SIAM J. Sci.

Comput. 26 (2004) 448-466.

X Jiang, S Mahadevan "Bayesian risk-based decision method for model validation under uncertainty", in Reliability Engineering System Safety (2007)

X. Jiang, S. Mahadevan, "Bayesian hierarchical uncertainty quantification by structural equation modeling", International Journal for Numerical Methods in Engineering, Volume 80, Issue 6-7, pages 717-737, 5 - 12 November 2009 
$\mathrm{X}$ Jiang, S Mahadevan, "Bayesian structural equation modeling method for hierarchical model validation", Reliability Engineering System Safety (2009), Volume: 94, Issue: 4, Pages: 796-809

X. Jiang, S. Mahadevan, A. Urbina, "Bayesian nonlinear structural equation modeling for hierarchical validation of dynamical systems", Mechanical Systems and Signal Processing (2010) Volume: 24, Issue: 4, Publisher: Elsevier, Pages: 957-975

Kang, S., Roy, R.P., 2002, Vapor Phase Measurements in Subcooled Boiling Flow, Journal Heat Transfer, 124, 1207-1209.

Z.E. Karoutas, Y. Sung, Y.R. Chang, G.A. Kogan, P.F. Joffre, "Subcooled Boiling Data From Rod Bundles", Paper no. ICONE12-49492 pp. 775-794, 12th International Conference on Nuclear Engineering (ICONE12) April, 2004 , Arlington, Virginia, USA).

Z. E. Karoutas, “Challenge Problem Technical Specification”, CASL Report, 2010

B. Kendrick, C. Stanek, and M. Short, "MAMBA (MPO Advanced Model for Boron Analysis) Development for CASL: update on the 3D v2.0 release", CASL communication, February 2012.

D.L. Keely and C. Smith, "Bayesian Inference for Probabilistic Risk Assessment", Springer, 2011, 224p.

M. C. Kennedy, A. O'Hagan, "Bayesian Calibration of Computer Models (with discussion),” J. R. Statist. Soc. B 63 (2001) 425-464.

E. Krepper, B. Koncar, and Y. Egorov, "CFD modelling of subcooled boilingConcept, validation and application to fuel assembly design", Nuclear Engineeering and Design, V.237, N.7, Apr 2007, p.716-731.

Kumar, R., Trabold, T.A., Maneri, C.C., 2003, Experiments and Modelling in Bubbly flows at elevated Pressure, J. Fluids Engineering, 125, 469-478.

H. Lee, "Development, Management and Utilization of Databases to Support Code V\&V”, Nuclear Engineering Summer School MeV-2010, Idaho Falls, July 2010

Lee, T.H., Park, G.C., Lee, D.J., 2002, Local Flow Characteristics of Subcooled Boiling of Water in a Vertical Concentric Annulus, Int. J. Multiphase Flow, 28, 1351-1368.

Luke, A. and Cheng., D., 2006, High Speed Video Recording of Bubble Formation with Pool Boiling, Int. J. Thermal Sciences, 45, 310-320.

S. Mahadevan and R. Rebba, "Validation of reliability computational models using Bayes networks", Reliability Engineering System Safety, V.87 (2), February 2005, Pages 223-232 
Maurus, R., Sattelmayer, T., 2006, Bubble and Boundary Layer Behaviour in Subcooled Flow Boiling, Int. J. of Thermal Sciences, 45, 257-268.

R. Montgomery, "Nuclear Fuel Performance, Simulation, and UQ", SAMSI, September 2011.

K. Mousseau, W. Ren, H. Lee, "CASL Data Center: Proposal for the Initial Design, Implementation and Demonstration", March 2012 (internal communication).

Myers, J.G., Yerramilli, V.K., Hussey, S.W., Yee, G.F., Kim, J., 2005, Time and Space Resolved Wall Temperature and Heat Flux Measurements during Nucleate Boiling with Constant Heat Flux Boundary Conditions, Int. J. Heat Mass Transfer, 48, 2429-2442.

R.A. Nelson, C. Unal, J. Stewart, B.J. Williams, 2010. 'Using Error and Uncertainty Quantification to Verify and Validate Modeling and Simulation Capabilities", Los Alamos National Laboratory report, LA-UR-10-06125.

R. Nourgaliev, N. Dinh, and R. Youngblood, 'Development, Selection, Implementation and Testing of Architectural Features and Solution Techniques for Next Generation of System Simulation Codes to Support the Safety Case if the LWR Life Extension", INL/EXT-10-19984, Idaho National Laboratory Report, September 2010.

R. R. Nourgaliev, N. Dinh, and R. Youngblood, "Risk-Informed Safety Margin Characterization", RELAP International Users Group Annual Meeting, September 21-23, 2010, West Yellowstone, Wyoming.

R.R. Nourgaliev and N. Dinh, "An Initial VU-Assessed Code Development Effort”, INL/LTD-10-20668, 236 p.

NUREG-1737: Software Quality Assurance Procedures for NRC Thermal Hydraulic Codes. December 2000.

W.T. Nutt, G.B. Wallis, "Evaluation of nuclear safety from the outputs of computer codes in the presence of uncertainties," Reliability Engineering \& System Safety, Vol. 83, pp. 57-77, (2004).

W. L. Oberkampf, T. G. Trucano and C. Hirsch. Verification, validation, and predictive capability in computational engineering and physics. Technical report, SAND2003-3769, Sandia National Laboratories, 2003.

W.L. Oberkampf, M. Pilch, T.G. Trucano, "Predictive Capability Maturity Model for Computational Modeling and Simulation," SAND2007-5948, October 2007.

W. L. Oberkampf and T. G. Trucano, "Verification and Validation Benchmarks," Sandia National Laboratories Technical Report, SAND2007-0853.

W. L. Oberkampf and C. J. Roy, Verification and Validation in Scientific Computing, Cambridge University Press, Cambridge, 2010. 
OECD-NEA, International Fuel Performance Experiments (IFPE) Database (2010) http://www.oecd-nea.org/science/fuel/ifpelst.html

OECD-NEA, "NUPEC BWR Full-size Fine-mesh Bundle Test (BFBT) Benchmark", NEA/NSC/DOC(2007)21, 2007

OECD/NRC, Benchmark based on NUPEC PWR Sub-channel Bundle Tests, (A. Rubin, A. Schoedel, M. Avramova): Vol. I: Experimental Database and Final Problem Specifications, NEA/NSC/DOC(2010)1 (November 2010)

Ohtake, H., Wada, N. and Koizumi, Y., 2003, Surface Roughness Effects on Onset of Nucleate Boling and Net Vapor Generation Point in subcooled Flow Boiling, 10th Int. Topical Meeting on Nuclear Reactor Thermal-Hydraulics (NURETH-10), Seoul, Korea, October 5-9.

Okawa, T., Ishida, T., Kataoka, I, Mori, M., 2005a, An Experimental study on Bubble rise Path after the Departure from a Nucleation Site in Vertical Upflow Boiling, Exp. Thermal and Fluid Science, 29, 287-294.

Okawa, T., Ishida, T., Kataoka, I, Mori, M., 2005b, On the Rise Path of single Vapor Bubbles after the Departure from Nucleation Sites in Subcooled Upflow Boiling, Int. J. Heat Mass Transfer, 48, 4446-4459.

A. Prosek and B. Mavko, "The State-of-the-Art Theory and Applications of BestEstimate Plus Uncertainty Methods," Nuclear Technology, Vol. 158, pp. 69-79, (2007).

M. Raffel, C.E. Willert, S.T. Wereley, and J. Kompenhans, Particle Image Velocimetry. Springer- Verlag, Berlin Heidelberg, 1998.

P. J. Roache. Verification and validation in computational science and engineering. Hermosa, 1998.

Roy, R.P., Velidandla, V., Kaira, S.P., Peturaud, P., 1994, Local Measurements in the Two-Phase Region of Turbulent Subcooled Boiling Flow, Journal Heat Transfer, $116,660-669$.

Roy, R.P., Kang, Velidandla, V., Kaira, S.P., 1997, Velocity Filed in Turbulent Subcooled Boiling Flow, Journal Heat Transfer, 119, 754-766.

Roy, R.P., Kang, S., Zarate, J.A., Laporta, A., 2002, Turbulent Subcooled Boiling Flow - Experiments and Simulations, Journal Heat Transfer, 124, 73-93.

Sadatomi, M., Kawahara, A., Kano, K., Sumi, Y., 2004, Single- and Two-Phase Mixing Rate between Adjacent Subchannels in a Vertical 2x3 Rod Array Channel, Int. J. Multiphase Flow, 30, 481-498. 
S. Sankararaman, and S. Mahadevan, "Model validation under epistemic uncertainty", Reliability Engineering \& System Safety, Volume 96, Issue 9, September 2011, Pages 1232-1241

J.A. Sawicki, "Analyses of crud deposits on fuel rods in PWRs using Mössbauer spectroscopy", Journal of Nuclear Materials 402 (2010) 124-129.

J. Secker and Z. Karoutas, “CRUD Challeneg Problem: Product Integration”, March 2012 (internal communication)

Situ, R., Hibiki, T., Sun, X., Mi, Y., Ishii, M., 2004, Flow structure of subcooled boiling in an internally heated annulus, Int. J. Heat Mass Transfer, 47, 5351-536.

Situ, R., Hibiki, T., Ishii, M., Mori, M., 2004b, Photographic study of bubble behaviours in Forced Convection Subcooled Boiling, Int. J. Heat Mass Transfer, 47, 3659-3667

T.G. Theofanous, "On the Proper Formulation of Safety Goals and Assessment of Safety Margins for Rare and High-Consequence Hazards", J. Reliability Engineering \& Systems Safety, 54, 1996, 243-257.

T.G. Theofanous, J.P. Tu, A.T. Dinh and T.N. Dinh, "The Boiling Crisis Phenomenon - Part 1: Nucleation and Nucleate Boiling Heat Transfer", J. Experimental Thermal and Fluid Science, pp.775-792, V.26 (6-7);- Part 2: Dryout Dynamics and Burnout”, J. pp.793-810, V.26 (6-7), August 2002.

T.G. Theofanous, and T.N. Dinh, "High Heat Flux Boiling and Burnout as Microphysical Phenomena: Mounting Evidence and Opportunities", Multiphase Science and Technology. 18(1), pp.1-26, 2006.

T.G. Theofanous and T.N. Dinh, "On the Prediction of Flow Patterns as a Principal Scientific Issue in Multifluid Flow", Multiphase Science and Technology. 15(1-4), pp.57-76, 2003.

T.G. Theofanous and T. N. Dinh, "Integration of Multi-Phase Science and Technology with Risk Management in Nuclear Power Reactors: Application of the Risk Oriented Accident Analysis Methodology to the Economic, Simplified Boiling Water Reactor Design”, Multiphase Science and Technology, v.20(2), pp.91-223, 2008.

B. H. Timmins, B. L. Smith, and P. P. Vlachos, "Automatic Particle Image Velocimetry Uncertainty Quantification,” ASME Fluids Engineering Conference, August, 2010, Paper number 2010- 30724.

Thorncroft, G.E., Klausner, J.F. and Mei., R., The Influence of Vapor Bubble Sliding in Forced convection Boling Heat Transfer, J. Heat Transfer, 121, 73-79. 1998.

J.P., Tu, T.N. Dinh, T.G. Theofanous, "Enhancing Resistance to Burnout via Coolant Chemistry", 10th International Topical Meeting on Nuclear Reactor Thermal Hydraulics NURETH-10, Seoul, Korea, 2003. 
J.P. Tu, T.N. Dinh and T.G. Theofanous, "An Experimental Study of Boiling Heat Transfer with Nanoparticles", 6th International Symposium on Heat Transfer, Beijing, June 2004.

Tu, J.Y., Yeoh, G.H., Park, G.C., Kim, M.O., On Population Balance Approach for Subcooled Boiling Flow Prediction, J. Heat Transfer, 127, 253-264. (2005)

J.A. Turnbull, Review of Nuclear Fuel Experimental Data - Fuel Behaviour Data Available from IFE-OECD Halden Project for Development and Validation of Computer Codes, NEA, 67p. 1995.

J.A. Turner, "Computational Science Challenges in Nuclear Energy", $2^{\text {nd }}$ Workshop on "Frontiers in Multi-Core Computing", UMD, September 2010.

Ünal, H.C., Maximum Bubble Diameter, Maximum Bubble-Growth Time and bubble -Growth Rate during the Subcooled Nucleate Flow Boling of Water up to 17.7 MN/m2, Int. J. Heat Mass Transfer, 19, 643-649. (1976)

C. Unal, B. Williams, D. Higdon, R.A. Nelson, “Towards Standardizing Uncertainty Estimations in Reactor Safety," Los Alamos National Laboratory report LA-UR-068642, ICAPP 2007, Paper 7082 May 13-18, Nice France, 2007 ANS summer meeting, Boston.

C. Unal, B. Williams, F. Hemez, S.H. Atamturktur, P. McClure, "Improved best estimate plus uncertainty methodology, including advanced validation concepts, to license evolving nuclear reactors", Nuclear Engineering and Design, Volume 241, Issue 5, May 2011, pp.1813-1833

G. Wang, W. A. Byers, M. Y. Young, and Z.E. Karoutas, "Westinghouse Advanced Loop Tester (WALT) Update", Paper no. ICONE16-48480, 16th International Conference on Nuclear Engineering (ICONE16), May 11-15, 2008 , Orlando, Florida, USA.

Warrier, G.R., Basu, N. and Dhir, V., 2002, Interfacial Heat Transfer during Subcooled Flow Boling, Int. J. Heat Mass Transfer, 45, 3947-3959

R.L. Williamson, J.D. Hales, S.R. Novascone, M.R. Tonks, D.R. Gaston, C.J. Permann, D. Andrs, R.C. Martineau, "Multidimensional multiphysics simulation of nuclear fuel behavior", Journal of Nuclear Materials, Volume 423, Issues 1-3, April 2012, Pages $149-163$.

G.E. Wilson and B.E. Boyack, "The Role of the PIRT Process in Experiments, Code Development and Code Applications Associated with Reactor Safety Analysis," Nuclear Engineering and Design, Vol. 186 pp. 23-37, (1998).

G.E. Wilson et al. " Quantifying Reactor Safety Margins Part 2: Characterization of important contributors to uncertainty," 119 (1990) 17-31.

W. Wulf et al. "Quantifying Reactor Safety Margins Part 3: Assessment and ranging of parameters, "Nuclear Engineering and Design 119 (1990) 33-65 
Yeoh, G.H., Tu, J.Y., A Bubble Mechanistic Model for Subcooled Boling Flow

Predictions, Numerical Heat Transfer, Part B, 45, 475-49; (2004)

Yeoh, G.H., Tu, J.Y., Thermal-Hydrodynamic modeling of Bubbly Flows with Heat and Mass Transfer, AIChE J., 51, 8-27. (2005a)

Yeoh, G.H., Tu, J.Y., A Unified Model considering Force Balances for Departing Vapour Bubbles and Population Balance in Subcooled Boling flow, Nucl. Eng. and Design, 235, 1251-1265. (2005b)

M.Y. Young, S. M. Bajorek, M. E. Nissley, and L. E. Hochreiter, "Application of Code Scaling Applicability and Uncertainty Methodology to the Large Break Loss of Coolant," Nuclear Engineering and Design, Vol. 186, pp. 39-52, (1998).

R. W. Youngblood, V. A. Mousseau, D. L. Kelly, and T.-N. Dinh, "Risk-Informed Safety Margin Characterization (RISMC): Integrated Treatment of Aleatory and Epistemic Uncertainty in Safety Analysis", International Conference on Nuclear Thermal Hydraulics, Operation, and Safety, NUTHOS-8, Shanghai, China, October $10-14,2010$

N. Zuber, "The Effects of Complexity, of Simplicity and of Scaling in ThermalHydraulics," Nuclear Engineering and Design, Vol. 204, n 1-3, pp 1-27, (2001). 


\section{Appendix A}

\section{Remarks on Sensitivity and Uncertainty Analysis for CIPS}

Techniques to support sensitivity and uncertainty analysis in computer simulations have been advanced over past three decades. Most theoretically established and software-ready are methods of statistical ("black-box", sampling-based) approach, which has been applied in study of AMS including AMS of complex physical systems. Deterministic ("glass-box") approach such as forward and adjoint sensitivity analysis procedures have also been developed. Notably, adjoint methods are successfully applied in the field of reactor physics/core loading analysis, which features a larger number of parameters (cross-section data), but otherwise static and linear systems. Deterministic and statistical methods alike, most analyses reported to date are performed to determine local sensitivities, by characterizing the impact of the input factors variation on the model (system response) in a sufficiently small neighborhood around a chosen nominal point in a static system, or around a transient solution in dynamic systems.

Under CASL support, deterministic approach (e.g., the adjoint sensitivity method) is geared to make important inroads in VERA-AC codes for radiation transport (DENOVO code) and computational fluid dynamics (DREKAR code). Progress notwithstanding, substantial efforts are still required to bring the adjoint sensitivity formulation to the domain of multi-physics problems and to demonstrate its practical (computationally affordable) implementation for reactor transients spanning a range of time scales in the CIPS problem.

Note that nuclear reactor engineering challenge problems like CIPS are non-linear (even just the thermal-hydraulics component) and transient, not to mention multi-physics and multi-scale nature in advanced models for CIPS processes. To characterize such systems, one wishes to be guided by sensitivity analysis in which the effect of an input variable on the model outcome is assessed globally and holistically, i.e.

i. incorporating the effect of the range and (pdf) distribution of the input variables, and recognizing that the input variation occurs

ii. when all other variables are varying, and

iii. all other parts (physics, scale) of the whole model are active as well.

Consequently, global analysis can provide invaluable information about the system behavior's "flow regime map" with transition boundaries and 
critical points (e.g., bifurcation). Development of methods and tools for global sensitivity analysis is in its infancy. While more straightforwardly extended to global analysis and successfully demonstrated (including in CASL) for smallscale problems, statistical approach requires a large number of simulation runs, which remain computationally prohibitive in advanced, high-fidelity modeling of multi-physics, multi-scale systems. Efforts to address this challenge include work on response surface / surrogate / reduce-order and hybrid methods.

As the CASL spearheads a paradigm shift toward an VUQ-enable nuclear engineering, a broader range of efforts to develop and apply modern VUQ methods and tools is welcomed. VUQ applications, including in a study subset of CIPS' decomposed (to single physics) and linearized problems, will help pave the way to learn, enrich the VUQ machinery and institute a new practice. Selection of the above-mentioned study subset should facilitate the development in respective topics, e.g. components of CIPS. However, while necessary and valuable on their own rights, such efforts are not sufficient for supporting the CASL mission on challenge problems. It is important for CASL to plan a case study (initially being heuristic but increasingly substantiated) to a qualitative "global analysis" of CIPS. This should take into account the CIPS' multi-physics multi-scale nature, and data heterogeneity. The probabilistic integration framework discussed in Section 4.2 .2 can be used for the case study. Another important benefit of taking on the CIPS as a whole is that it will serve as platform for testing and bringing to convergence different practices in data qualification as discussed in Section 3.2.3. 